\title{
Acalyptris Meyrick: revision of the platani and staticis groups in Europe and the Mediterranean (Lepidoptera: Nepticulidae)
}

\section{ERIK J. VAN NIEUKERKEN}

National Museum of Natural History, Naturalis, PO Box 9517, NL-2300 RA Leiden, The Netherlands.

E-mail: nieukerken@naturalis.nl

\section{Table of contents}

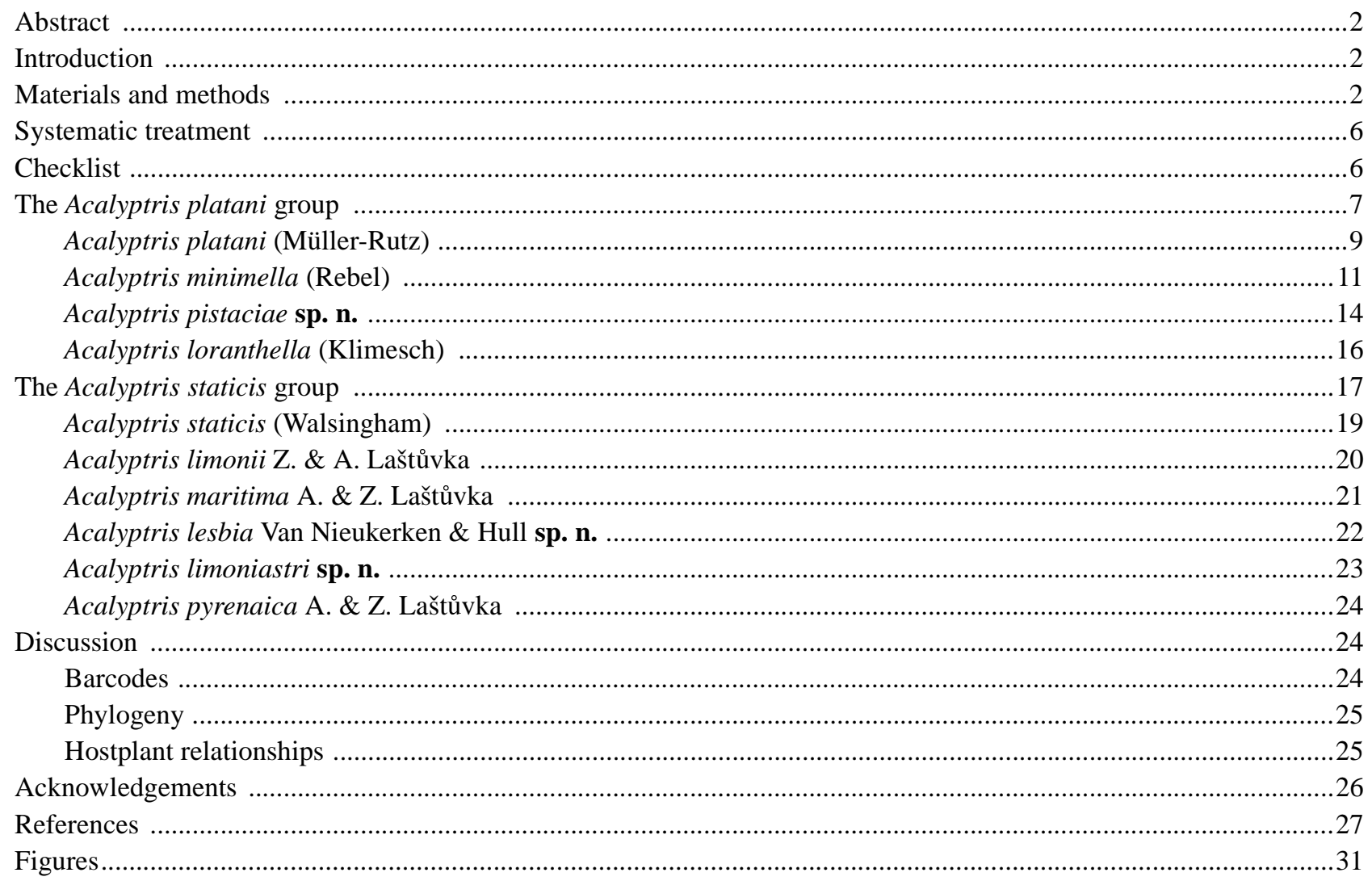




\begin{abstract}
The European and Mediterranean species of the Acalyptris platani and A. staticis groups are revised, with respectively four and six species. Three new species are described: A. pistaciae Van Nieukerken sp. n. (from Greece, Cyprus and Turkey, on Pistacia) in the platani group, A. limoniastri Van Nieukerken sp. n. (from Algeria and Tunisia, associated with Limoniastrum) and A. lesbia Van Nieukerken \& Hull sp. n. (from Greece: Lesvos, on Limonium gmelini) in the staticis group. Lectotypes are selected for A. minimella (Rebel, 1924) and A. staticis (Walsingham, 1908). The European species of the A. platani group are leafminers on Anacardiaceae, Platanaceae and Loranthaceae, the species of the A. staticis group feed on Plumbaginaceae, except A. pyrenaica A. \& Z. Laštůvka, for which the host is unknown, but likely to belong to another family. Immatures are described for five species, final instar larvae and pupal exuviae for all species in the A. platani group and for A. lesbia. Larvae and pupae of the latter species differ markedly from the A. platani group. Recorded parasitoids are listed. CO1 barcodes are provided for seven species. The groups are phylogenetically not very close; the monophyly of Acalyptris and the phylogeny on the basis of CO1 (mt-DNA) sequences are discussed.
\end{abstract}

Key words: Taxonomy; new species; phylogeny; larval morphology; pupal morphology; hostplants; Anacardiaceae; Platanaceae; Loranthaceae; Plumbaginaceae; CO1; DNA-barcodes; Palaearctic; Hymenoptera; Eulophidae; Braconidae

\title{
Introduction
}

The Nepticulidae genus Acalyptris Meyrick, 1921 is particularly diverse in tropical regions, and well known from South Africa and the Neotropics, with respectively 23 and 21 named species (Scoble 1980; van Nieukerken 1986a; Puplesis et al. 2002b). It is also abundant in desert and steppe regions of the Old World with ca. 16 named species (Puplesis 1990; Puplesis \& Diškus 1995), fewer are known from North America (Wilkinson 1979), but several undescribed species are present in collections (D.R. Davis, personal communication). Only seven species are listed for Europe and the Canary Islands (van Nieukerken 2004), five of which were included in the key work by A. \& Z. Laštůvka (1997). The genus is classified in the tribus Trifurculini, in the subfamily Nepticulinae.

Acalyptris is a heterogeneous genus, and especially variable in the structure of the genitalia. Its present composition was established by van Nieukerken (1986a) by synonymizing the genera Microcalyptris Braun, 1925 and Niepeltia Strand, 1934 with Acalyptris. The only apomorphy listed by van Nieukerken (1986a) is: "Closed cell in forewing shifted towards base, vestigial" (Figs. 18, 19).

A division of Acalyptris into species groups was first undertaken by Puplesis (1994), who recognized the repeteki and shafirkanus group. Later Diškus and Puplesis (2003) erected also the Neotropical latipennata group, but listed the species that were previously placed in the shafirkanus group under "species not assigned to group". The platani and staticis groups were briefly discussed by van Nieukerken \& Biesenbaum (1997) and are further established here. Apart from these two, no other groups occur in Europe. In North Africa and the Near East also species belonging to the repeteki and shafirkanus groups occur (van Nieukerken, unpublished information). Because of the large morphological and biological variation in the genus, in addition to uncertainties about monophyly, I refrain from a generic description, but provide group diagnoses instead.

Three new species are described here. The immature stages of this genus are reviewed for the first time for all species in the platani group and for A. lesbia sp. n. in the staticis group.

\section{Materials and methods}

Material. For the collections the abbreviations (codons) from Evenhuis \& Samuelson (2004) are used, with the following additions: 
CVDB C. van den Berg collection (Hoogland, Netherlands)

GB G. Bassi collection (Torino, Italy)

MC M. F. V. Corley collection (Faringdon, United Kingdom)

MH M. Hull collection (Gwynned, United Kingdom)

MHNM Museum d'Histoire Naturelle de Marseille (France) (containing collection of the late R. Buvat)

PT P. Triberti collection (Verona, Italy)

RJ R. Johansson collection (Växjö, Sweden), to be deposited in ZMUC

RS R. Sutter collection (Bitterfeld, Germany)

VPU Vilnius Pedagogical University, Department of Zoology (Vilnius, Lithuania)

WB W. Biesenbaum collection (Velbert-Langenberg, Germany)

WML World Museum Liverpool (United Kingdom)

Microscope slides and the associated specimens in the collection of RMNH receive a RMNH registry number (RMNH-INS no.), which for slides prepared by the author can be calculated by adding 20,000 to his EvN or VU slide number. All material and literature data are databased in the authors' database of material; an Excel version of the data in this paper, including coordinates, is available on the author's website (http:// www.naturalis.nl/nieukerken). Data for specimens examined are arranged alphabetically by country and locality; when applicable, the province name is given as the first item in the locality. Depositories are only given after the last record in a row, when more than one record refers to the same collection. In the material lists, examined adults and larvae are mentioned first, and leafmines are listed separately after that. Unpublished records received from colleagues are listed under "Additional records".

Material used for molecular analysis is listed in Table 1, with deposition of vouchers and Genbank accession numbers. When adults were used, vouchers comprise the dry adult in the collection and the associated genitalia slide, details under material examined. When larvae were used, in most cases the voucher consists of the associated leafmine, in two cases the larval skin was kept and slide mounted. No vouchers exists for $A$. loranthella.

\section{Methods}

Morphology. Genitalia preparations were embedded in Euparal, following the methods described in van Nieukerken et al. (1990), for recent material, genitalia preparation and DNA extraction was combined, see below. Larvae and pupal exuviae were prepared in a similar fashion, after maceration in $\mathrm{KOH}$, and usually embedded in Euparal, but in the early 1980's partly also embedded in Polyvinyl Lactophenol. Such slides, however, are prone to deterioration (Brown 1997). Larval slides were either unstained, or stained with both Chlorazol Black and Haemaluin (acc. to Mayer) and in some recent slides several other stains or combinations thereof have been used; pupal exuviae were stained with Chlorazol Black and Acid Fuchsin or Phenosaffra$\operatorname{nin}$.

Measurements of genitalia and larvae were obtained from digital images, using AxioVision, 20× objective for male genitalia and usually $10 \times$ for females. Some older measurements were taken with an eyepiece micrometer, with a precision of about $5 \mu \mathrm{m}$. Capsule length was measured from middle of vinculum to middle of uncus, excluding any projections; valva length from tip of posterior process to ventral edge, not the sublateral process; aedeagus length was measured from the sclerotized tube, excluding any protruding vesica parts. Bursa length is measured from cloaca to anterior tip. Forewing length was measured from tip of cilia to attachment on thorax, usually at magnification of $20 \times$. Antennal segment counts include scape and pedicel. The larval headcapsule length was measured excluding the mouthparts. Measurements of moths are presented in table 2, those of male genitalia in table 3, measurements of female genitalia and larvae are given in the text.

Morphological terms follow van Nieukerken et al. (1990). 


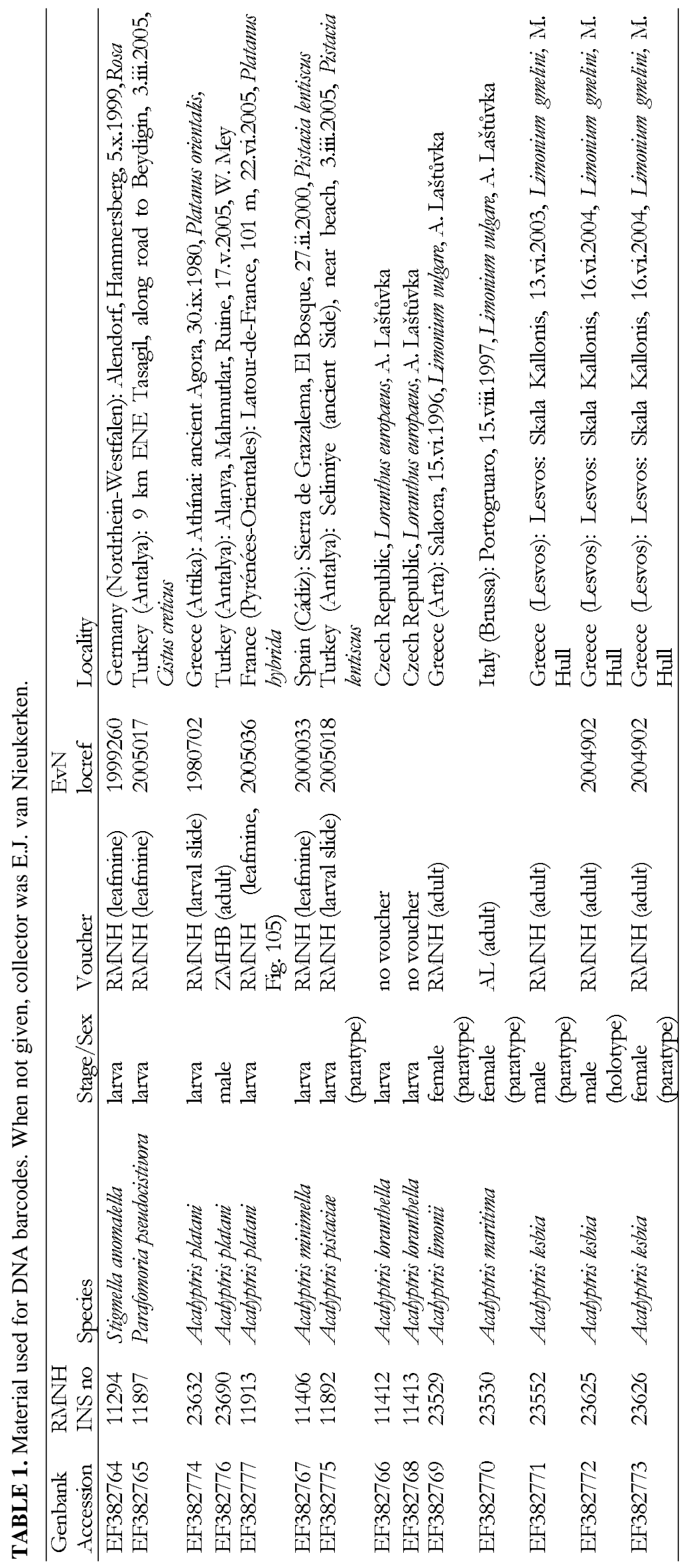


Molecular methods. DNA was extracted from caterpillars or from dry adult abdomens. DNA extraction from larvae was usually destructive, from abdomens and some larvae the non-destructive protocol by Knölke et al. (2005) was followed, allowing the preparation of the genitalia or larval skin as well. No suitable material of A. limoniastri, A. staticis, or A. pyrenaica was available for DNA extraction. DNA was extracted by use of the Qiagen Dneasy Tissue Kit, following manufacturer's protocol. Elution volume was $75 \mu 1$. A 665 bp fragment of the 5' end of the CO1 (Cytochrome C Oxidase subunit 1) mitochondrial gene, the DNA barcode, was amplified, by using forward primer LEP-F1 (5'-ATTCAACCAATCATAAAGATAT-3') and reverse primer LEP-R1 (5'-TAAACTTCTGGATGTCCAAAAA-3') (Hebert et al. 2004).

PCR conditions were $2.5 \mathrm{mM} \mathrm{MgCl}, 0.2 \mathrm{mM}$ DNTPs, $0.4 \mu \mathrm{M}$ of each primer and $5 \mathrm{U}$ of Taq DNA polymerase (Qiagen) per $25 \mu \mathrm{l}$ reaction. Thermocycle conditions were $1 \mathrm{~min}$. at $94^{\circ} \mathrm{C}$, followed by six cycles of 1 min. at $94^{\circ} \mathrm{C}, 1 \mathrm{~min}$. and $30 \mathrm{sec}$. at $45^{\circ} \mathrm{C}$ and $1 \mathrm{~min}$. and $15 \mathrm{sec}$. at $72^{\circ} \mathrm{C}$, followed by 36 cycles of $1 \mathrm{~min}$. at $94^{\circ} \mathrm{C}, 1 \mathrm{~min}$. and $30 \mathrm{sec}$. at $51^{\circ} \mathrm{C}$ and $1 \mathrm{~min}$. and $15 \mathrm{sec}$. at $72^{\circ} \mathrm{C}$, with a final extension step of $5 \mathrm{~min}$. at $72^{\circ} \mathrm{C}$. Except for the pcr-products of the outgroup taxa Stigmella anomalella and Parafomoria pseudocistivora, all sequencing reaction were done by Macrogen inc. (Korea). Sequencing reactions were conducted under Big Dye $^{\mathrm{TM}}$ termination conditions. Reacted products were purified using ethanol precipitation and run on an ABI 3730 automated sequencher (Applied Biosystems). The pcr-products of S. anomalella and P. pseudocistivora were obtained during a prior study and were directly purified using a Nucleospin® Extract II kit (MacheryNagel). Sequencing was done in both directions and took place under the same conditions. Reaction products were dye-terminator cycle sequenced (Big Dye kit by Applied Biosystems) in both directions using manufacturer's protocol and purified using AutoSeq ${ }^{\mathrm{TM}}$ G-50 columns (Amersham Biosciences); they were run on an ABI377 automated sequencer (Applied Biosystems). Forward and reverse sequences were assembled and checked using Sequencher 4.0 (Gene Codes corporation). Obtained sequences were aligned manually using BioEdit (Hall 2004).

Phylogenetic analyses. Phylogeny was only analysed on the basis of the partial mitochondrial CO1 gene. As outgroup I used Stigmella anomalella (Goeze, 1783), a member of the tribe Nepticulini, and Parafomoria pseudocistivora Van Nieukerken, 1983 as a member of the Trifurculini, the genus Parafomoria being the possible sistergroup of Acalyptris, based on preliminary analyses of a larger sample of the family and three genes. A parsimony analysis and neighbour joining analysis were carried out with Paup 4.0b10 for Windows (Swofford 2001). The molecular dataset consists of only 14 sequences, two of which are redundant. The sequences of Acalyptris loranthella (RMNH 11412 \& 11413) and two of A. lesbia (RMNH 23625 \& 23626) are identical. The redundant sequences were deleted from the dataset before parsimony analysis, allowing an exhaustive search for the most parsimonious trees of the 12 remaining taxa. I used the Kimura 2 parameter as distance criterion for the neighbour joining tree and pairwise distances calculations.

Illustrations. Photographs of moths, leafmines, and microscopic slides (genitalia, larvae, pupae) were taken with a Zeiss AxioCam (HR) digital camera attached to a Zeiss Stemi SV11 stereo-microscope or a Zeiss Axioskop H, using Carl Zeiss AxioVision software. Some photographs of leafmines were obtained with a Canon EOS350 digital camera. Manipulation of photographs, using Adobe Photoshop® was kept to a minimum: conspicuous shades, protruding parts of pins, dust and air bubbles in slides were removed or obscured. SEM micrographs were made in 1980-1984 at the Free University, Amsterdam, with an ISI-40 scanning electron microscope, with a beam current of 5 or $10 \mathrm{kV}$ on gold coated specimens. Larvae were dried with critical point drying using Amyl-acetate and $\mathrm{CO}_{2}$, adults were air dried. Some of the SEM micrographs presented here, were published before in the context of family treatments (van Nieukerken 1986a; van Nieukerken \& Dop 1987; van Nieukerken et al. 1990).

Drawings of genitalia were made with a drawing tube attached to various Zeiss microscopes; the drawing of the aedeagus of A. pyrenaica has previously been published by van Nieukerken \& Biesenbaum (1997). During mock up of the plates, I have attempted to have all illustrations on one plate in the same scale; this was, however, not possible for the plates with adults. 
Distribution maps are prepared with DMAP 7.0 (Morton 2000), based on the examined material supplemented with the data from the original descriptions and other reliable literature records, and records provided by B. Gustafsson, J. Buszko, and A. and Z. Laštůvka. UTM coordinates or longitude/latitude when not given on labels or in references, were mainly derived from the GEOnet Names Server (NIMA 2004). Fig. 125 also shows the approximate distribution of the hostplant Loranthus europaeus as a shaded area. Nomenclature of hostplants follows Tutin et al. (1964-1993).

TABLE 2. Measurements of adult Acalyptris. Mean, standard deviation and sample size are given in brackets, for sample size 3 or smaller only sample size is provided.

\begin{tabular}{llll}
\hline Species & male forewing length & female forewing length & male wingspan \\
\hline A. platani & $2.4-2.5 \mathrm{~mm}(2.4 \pm 0.1,5)$ & $2.3-2.4 \mathrm{~mm}(2.4 \pm 0.1,6)$ & $5.2-5.4 \mathrm{~mm}$ \\
A. minimella & $1.8-2.4 \mathrm{~mm}(2.2 \pm 0.2,13)$ & $2.1-2.6 \mathrm{~mm}(2.3 \pm 0.1,11)$ & $4.1-5.4 \mathrm{~mm}$ \\
A. pistaciae & $1.8-2.4 \mathrm{~mm}(2.0 \pm 0.2,12)$ & $1.6-2.2 \mathrm{~mm}(1.9 \pm 0.2,10)$ & $4.0-5.3 \mathrm{~mm}$ \\
A. loranthella & $1.8-2.4 \mathrm{~mm}(2.1 \pm 0.2,16)$ & $2.1-2.5 \mathrm{~mm}(2.3 \pm 0.1,9)$ & $3.7-5.5 \mathrm{~mm}$ \\
A. staticis & $1.5-2.3 \mathrm{~mm}(2.0 \pm 0.2,8)$ & $1.9-2.3 \mathrm{~mm}(2.0 \pm 0.1,10)$ & $4.3-4.7 \mathrm{~mm}$ \\
A. limoniastri & $2.5-3.0 \mathrm{~mm}(2.8 \pm 0.2,10)$ & 2.96 & $5.5-6.6 \mathrm{~mm}$ \\
A. maritima & $2.3-2.6 \mathrm{~mm}(2.4 \pm 0.1,4)$ & $2.1 \mathrm{~mm}(1)$ & $5.0-5.5 \mathrm{~mm}$ \\
A. limonii & $1.9 \mathrm{~mm}(2)$ & $2.2-2.3 \mathrm{~mm}(2)$ & $4.1-4.3 \mathrm{~mm}$ \\
A. lesbia & $1.7-2.1 \mathrm{~mm}(1.8 \pm 0.1,12)$ & $1.9-2.2 \mathrm{~mm}(2.0 \pm 0.1,10)$ & $4.0-4.6 \mathrm{~mm}$ \\
A. pyrenaica & $2.0-2.5 \mathrm{~mm}(2.3 \pm 0.2,4)$ & - & $4.4-5.6 \mathrm{~mm}$ \\
\hline
\end{tabular}

continued.

\begin{tabular}{llll}
\hline Species & female wingspan & male antennal segments & female antennal segments \\
\hline A. platani & $5.0-5.4 \mathrm{~mm}$ & $34(3)$ & $27-29(28.6 \pm 0.9,5)$ \\
A. minimella & $4.7-5.8 \mathrm{~mm}$ & $32-39(35.4 \pm 2.1,8)$ & $28-33(29.7 \pm 1.4,10)$ \\
A. pistaciae & $3.7-4.9 \mathrm{~mm}$ & $27-33(30.8 \pm 1.8,9)$ & $20-30(24.3 \pm 3.7,8)$ \\
A. loranthella & $4.5-5.5 \mathrm{~mm}$ & $30-39(33.4 \pm 2.4,12)$ & $33-36(34.5 \pm 1.1,8)$ \\
A. staticis & $4.0-5.0 \mathrm{~mm}$ & $38-40(39.3 \pm 1.0,6)$ & $34-36(35.2 \pm 0.8,6)$ \\
A. limoniastri & $6.24 \mathrm{~mm}$ & $43-46(45.2 \pm 1.3,5)$ & $38(1)$ \\
A. maritima & $4.6-4.6 \mathrm{~mm}$ & $32-36(33.8 \pm 1.7,4)$ & $32(1)$ \\
A. limonii & $4.8-4.8 \mathrm{~mm}$ & $32(2)$ & $30-32(2)$ \\
A. lesbia & $4.4-4.8 \mathrm{~mm}$ & $28-34(31.6 \pm 1.7,11)$ & $29-31(30.3 \pm 1.0,9)$ \\
A. pyrenaica & - & $36-39(3)$ & - \\
\hline
\end{tabular}

\section{Systematic treatment}

\section{Checklist}

Acalyptris Meyrick, 1921

Type-species: Acalyptris psammophricta Meyrick, 1921 (original designation)

Microcalyptris Braun, 1925

Type-species: Microcalyptris scirpi Braun, 1925 (original designation)

Weberia Müller-Rutz, 1934 [a junior homonym of Weberia Robineau-Desvoidy, 1830]

Type-species: Weberia platani Müller-Rutz, 1934 (original designation) 
Niepeltia Strand, 1934 [15 August]

replacement name for Weberia Müller-Rutz

Weberina Müller-Rutz, 1934 [15 September]

replacement name for Weberia Müller-Rutz

platani group

platani (Müller-Rutz, 1934)

minimella (Rebel, 1924)

lentiscella (Groschke, 1944)

pistaciae sp. $\mathrm{n}$.

minimella sensu Klimesch 1978 partim

loranthella (Klimesch, 1937)

staticis group

staticis (Walsingham, 1908)

limonii Z. \& A. Laštůvka, 1998

maritima A. \& Z. Laštůvka, 1997

lesbia sp. n.

limoniastri sp. $\mathrm{n}$.

pyrenaica A. \& Z. Laštůvka, 1993

TABLE 3. Measurements of male genitalia of Acalyptris. Mean, standard deviation and sample size are given in brackets, for sample size 3 or smaller only sample size is provided.

\begin{tabular}{llllll}
\hline Species & capsule length & capsule ratio l:w & valva length & aedeagus length & $\begin{array}{l}\text { ratio aedeagus:cap- } \\
\text { sule length }\end{array}$ \\
\hline A. platani & $292-317 \mu \mathrm{m}$ & $1.2-1.3$ & $176-193 \mu \mathrm{m}$ & $321-346 \mu \mathrm{m}$ & $1.0-1.2$ \\
& $(303.1 \pm 11.4,4)$ & $(1.24 \pm 0.04,4)$ & $(184.4 \pm 7.9,4)$ & $(334.8 \pm 13.0,4)$ & $(1.11 \pm 0.07,4)$ \\
A. minimella & $257-313 \mu \mathrm{m}$ & $1.1-1.3$ & $184-201 \mu \mathrm{m}$ & $300-353 \mu \mathrm{m}$ & $1.1-1.3$ \\
& $(272.2 \pm 18.8,7)$ & $(1.19 \pm 0.06,4)$ & $(194.1 \pm 8.4,6)$ & $(327.9 \pm 21.3,5)$ & $(1.18 \pm 0.07,5)$ \\
A. pistaciae & $248-317 \mu \mathrm{m}$ & $1.3-1.4(3)$ & $164-197 \mu \mathrm{m}$ & $282-343 \mu \mathrm{m}$ & $1.0-1.3$ \\
& $(284.2 \pm 30.6,6)$ & & $(184.4 \pm 13.6,6)$ & $(315.6 \pm 23.6,6)$ & $(1.12 \pm 0.07,6)$ \\
A. loranthella & $269-272 \mu \mathrm{m}(2)$ & $1.2(2)$ & $172-182 \mu \mathrm{m} \mathrm{(2)}$ & $320-332 \mu \mathrm{m}(2)$ & $1.2(2)$ \\
A. staticis & $206-238 \mu \mathrm{m}$ & $1.2-1.3$ & $146-163 \mu \mathrm{m}$ & $163-193 \mu \mathrm{m}$ & 0.8 \\
& $(223.4 \pm 13.2,5)$ & $(1.25 \pm 0.04,5)$ & $(154.0 \pm 7.7,5)$ & $(179.9 \pm 10.9,5)$ & $(0.81 \pm 0.03,5)$ \\
A. limoniastri & $399-429 \mu \mathrm{m}$ & $1.5-1.7$ & $249-261 \mu \mathrm{m}(3)$ & $273-296 \mu \mathrm{m}$ & $0.6-0.7(3)$ \\
& $(415.4 \pm 15.5,4)$ & $(1.58 \pm 0.06,4)$ & & $(283.6 \pm 11.6,4)$ & \\
A. maritima & $260-302 \mu \mathrm{m}(2)$ & $1.3(1)$ & $180-181 \mu \mathrm{m} \mathrm{(2)}$ & $184-217 \mu \mathrm{m} \mathrm{(2)}$ & $0.7(2)$ \\
A. limonii & $325 \mu \mathrm{m}(1)$ & $1.6(1)$ & - & $239 \mu \mathrm{m}(1)$ & $0.7(1)$ \\
A. lesbia & $318-329 \mu \mathrm{m}(2)$ & $1.4(1)$ & $180-181 \mu \mathrm{m} \mathrm{(2)}$ & $248-251 \mu \mathrm{m} \mathrm{(2)}$ & $0.8(1)$ \\
A. pyrenaica & $269 \mu \mathrm{m}(1)$ & $1.4(1)$ & $243 \mu \mathrm{m}(1)$ & $186 \mu \mathrm{m}(1)$ & $0.7(1)$ \\
\hline
\end{tabular}

\section{The Acalyptris platani group}

This group comprises A. platani (type-species of the generic synonym Niepeltia) and its relatives. They are externally very variable moths, usually with elaborate secondary sexual scaling. Male genitalia are characterized by absence of the transverse bar of transtilla, valva usually with elaborate inner processes, vinculum with 
posterior ventral plate, joining to aedeagal carinae. Female genitalia have elaborate vaginal sclerites, and conspicuous elongate reticulate signa.

Previous descriptions of the genus Acalyptris (or Niepeltia) often refer primarily to this group (i.e. Scoble 1980; Gustafsson \& van Nieukerken 1990; van Nieukerken \& Johansson 1990).

Adult. Head: collar small, comprising hair-scales, or occasionally lamellar scales (not in Europe). Antennal morphology: sensillum vesiculocladum with 3-4 short branches (studied in A. platani and A. loranthella; van Nieukerken \& Dop 1987) (Fig. 98). Forewing with various colour patterns; cilia-line normally present, often indistinct. Venation (Fig. 18) forewing: closed cell shifted towards base, hardly visible, main trunk $\mathrm{R}_{\mathrm{s}}+\mathrm{M}$ approximately straight, with three branches: $\mathrm{R}_{2+3}, \mathrm{R}_{4+5}$ and $\mathrm{M} ; \mathrm{Cu}$ not present; hindwing as in most Nepticulidae with forked $\mathrm{R}_{\mathrm{s}}+\mathrm{M}$ and separate $\mathrm{Cu}$ and $\mathrm{A}$. Forewing underside with distinct subdorsal retinaculum (Fig. 18). Hindwing with costal bristles in male and female, these occasionally lost in male. Most species with special, androconial scales (Figs. 94-97). Male with anal tufts on T8. Sternite 2 with 2A trapezoid, wider than long (Fig. 61).

Male genitalia. Vinculum invisibly fused with tegumen, forming complete ring; ventral plate usually bilobed, often reduced to flimsy membrane. Tegumen forming variably shaped pseuduncus. Uncus with single medial process, divided dorsoventrally. Gnathos with single medial element. Valva more or less triangular or elongate, usually with prominent inner processes. Transtilla: transverse bar absent. Aedeagus with two pairs of carinae, the ventral pair fused to a ventral process or juxta; vesica with small denticulate cornuti and often additional larger sclerotisations. Aedeagus relatively long, ratio aedeagus : capsule 1.0 to 1.3 (Table 3).

Female genitalia. Terminalia blunt and broad. T8 a single plate, often narrow, with single setae or groups of setae. T9 comprising distinct pair of setose anal papillae. Vestibulum with elaborate sclerotisations and additional pectinations. Ductus spermathecae coiled, vesicle prominent. Corpus bursae long, with pectinations and long reticulate signa.

Immature stages. Larva with four instars. Body yellow. Final instar larva: headcapsule (Figs. 84-87) usually, but not always, wider than long. Frontoclypeus rectangular. Tentorial arms short, anterior approximately as long as posterior. Labrum with medial and lateral setae (Fig. 100). Labial palpi with two short segments and long apical seta. Antenna with sensilla not placed cross-wise (Van Nieukerken 1986a) (details of head Figs. 100, 101).

Prothorax with small ventral sclerite and narrow paired dorsal sclerites (Figs. 85, 86). Pro-, meso- and metathorax respectively with 13,11 and 10 pairs of setae. A1-8 with 6, A9 with 2 or 3 and A10 with 3 pairs of setae. Anal rods curved, posteriorly ending in 3 pointed tips (Fig. 89). Spinosity of thorax and abdomen variable.

Earlier instars. Setae absent, except A8 (3 pairs). Integument slightly spinose.

Pupa (studied from exuviae of platani, pistaciae and loranthella). Head: clypeus transverse; frons without setae, with conical projection (only seen in platani), completely detached at emergence (Fig. 93); labial palpi longer than maxillae. A2-8 dorsally with many relatively short spines, arranged in about 4-5 indistinct transverse rows per segment (Fig. 91); cremaster comprising two widely separate spines.

Biology. The members of this group feed on a wide variety of hostplants. In Europe they comprise Platanaceae, Anacardiaceae and Loranthaceae, but in other areas include also Brassicaceae, Combretaceae, Euphorbiaceae, Fabaceae, Lythraceae, Melastomataceae (Oriental Region and Australia, unpublished data van Nieukerken), Rhamnaceae, Rubiaceae, Rutaceae and Theaceae. In the terms of the phylogenetic classification of Angiosperms (APGII: Bremer et al. 2003) most of these families belong to the Rosids in the wide sense, but Platanaceae and Loranthaceae belong to more basal clades in the Eudicots and Rubiaceae and Theaceae belong to the Asterid clade.

All make gallery leafmines, the larvae feed in the mine with their ventral side uppermost and they quit the mines before making a cocoon amongst leaf litter. All the species treated here have more than one generation per year. 
Parasitoids. Parasitic Hymenoptera are regularly reared, the following species have been recorded from European Acalyptris species:

Eulophidae (nomenclature according to Noyes 2003):

Apotetrastichus sp., reared from A. minimella (Lo Duca et al. 2002).

Chrysocharis gemma (Walker, 1839), reared from A. minimella (Lo Duca et al. 2002).

Chrysocharis loranthellae Erdös, 1954, reared from A. loranthella in Hungary and Romania and A. platani in Italy (In Hansson stated as Stigmella sp. on Platanus, which can only be A. platani) (Tudor \& Drăghia 1978; Hansson 1985). As yet Acalyptris species are the only known hosts, although Erdös (1956) also reports a male reared from Quercus cerris leaves, but the host insect is still unknown.

Cirrospilus variegatus (Masi, 1907), reared from A. minimella in Sicily (Rizzo \& Massa 2002).

Cirrospilus vittatus Walker, 1838, reared from A. minimella in Spain and Sicily (Askew 1984; Lo Duca et al. 2002).

Citrostichus phyllocnistoides (Narayanan, 1960), reared from A. minimella in Sicily (Massa et al. 2001; Lo Duca et al. 2002; Rizzo \& Massa 2002).

Closterocerus formosa Westwood, 1833, reared from A. minimella in Sicily (Lo Duca et al. 2002).

Neochrysocharis chlorogaster (Erdös, 1966), reared from A. loranthella in Hungary (Hansson 1990).

Braconidae:

Gnamptodon breviradialis Fischer, 1959, reared from A. loranthella in Greece and Hungary (Szőcs 1979; van Achterberg 1983).

Mirax rufilabris Haliday, 1833, reared from A. minimella in Spain, Mallorca (incorrectly cited as Italy) (Papp 1989). According to C. van Achterberg (pers. comm.), Mirax rufilabris forms an incompletely understood complex of species, and this is probably not the 'real' rufilabris, which seems specialised on Trifurcula (see also van Nieukerken et al. 2004b).

Composition. Apart from the four European species treated here, all South-African species of Acalyptris (Scoble 1980), several species from the Oriental region (Puplesis \& Diškus 2003), Eastern Palaearctic (unpublished data), Neotropical region (Puplesis \& Robinson 2000; Puplesis et al. 2002a) and Australia (R.J.B. Hoare \& E.J. van Nieukerken, unpublished data) belong to this group.

\section{Acalyptris platani (Müller-Rutz)}

(Figs. 1, 2, 7, 20, 21, 45, 62, 84, 89, 93, 94, 95, 98, 102, 105, 122)

Weberia platani Müller-Rutz, 1934a (15 June): 122-123, pl. 1. Syntypes: SWITZERLAND, Tessin, Mendisio, x.1932, ex larva Platanus acerifolia [not examined].

Niepeltia platani; Strand 1934 (15 August): 241 [new combination].

Weberina platani; Müller-Rutz 1934b (15 September): separate paper slip, inserted in issue [new combination].

Niepeltia platani; Lhomme 1934: 138 [France]; Klimesch 1951: 66 [Italy]; Hering 1957: 789 [mine keys]; Utech 1962: 234 [Georgia]; Klimesch 1968: 195 [Macedonia]; Scoble 1980: 205 [redescription]; van Nieukerken 1982: 19 [Greece].

Weberina platani; Skala 1938: 31 [Corsica]; Buhr 1940: 209 [Corsica]; Klimesch 1940: 191 [Italy].

Trifurcula (Weberina) platani; Klimesch 1978: 253, fig. 35 [Greece].

Acalyptris platani; van Nieukerken 1986b: 141 [new combination, listed]; van Nieukerken \& Johansson 1990: 258 [description]; Gustafsson \& van Nieukerken 1990: 345 [larva described]; A. \& Z. Laštůvka 1993: 158; van Nieukerken 1996: 24 [catalogued]; A. \& Z. Laštůvka 1997: 117 [description]; Olivella 2000: 30 [Spain]; Wieser et al. 2002: 5 [Iran]; van Nieukerken et al. 2004a: 227 [Iberian Peninsula]; A. \& Z. Laštůvka 2005: 10 [Sicily]; van Nieukerken et al. 2006: 56 [France, Corsica].

Stigmella sp.; Hering 1957: 789 [leafmines with narrow frass]; Hansson 1985: 92 [parasite reared, Italy]. 
Diagnosis. Males unmistakeable by colour pattern and cuspidate hindwings with special raised scales, not resembling any other Nepticulidae. Female may somewhat resemble Ectoedemia (Etainia) species because of double fascia, but these have more prominent pattern and are usually larger (see van Nieukerken \& Laštůvka 2002). Male genitalia very characteristic, separated from the other species in the group by the valva with two inner lobes. Female genitalia with vaginal sclerotisations which are characteristic for the group, separated from the next three species by the more rounded abdominal tip and extremely narrow signa with crenate margins.

Description. Male (Figs. 1, 7). Forewing length 2.4-2.5 mm, wingspan 5.2-5.4 mm. Head: frontal tuft yellowish, on vertex fuscous, two areas clearly separated; collar very inconspicuous, as vertex; scape and pedicel white. Antenna with ca. 34 segments. Thorax yellowish white to brown. Forewing basal 1/4 yellowish brown, followed by broad yellowish white fascia to 1/2; distal part of wing brown, yellowish white opposite tornal and costal spots, sometimes united in a second fascia; cilia silvery white, cilia line distinct. Underside forewing basal $2 / 3$ (in rest in contact with hindwing androconial patch) pale, with very few elongate brown scales only, distal third brown. Hindwing very broad at base, clearly cuspidate towards tip; costal bristles present; upper surface of basal $2 / 3$ covered with a thick indumentum of white raised special scales, interrupted along midline by a distinct, straight, longitudinal furrow, devoid of scales (Figs. 7, 94); costa with a row of short yellowish brown hair scales instead of cilia. Underside white. Abdomen with yellow inconspicuous anal tufts.

Female (Fig. 2). Forewing length 2.3-2.4 mm, wingspan 5.0-5.4 mm. Antenna with 27-29 segments. Forewing underside pale grey-brown, normally covered with scales. Hindwing normal, without special scales.

Male genitalia (Figs. 20, 21, 62). Measurements: see Table 3. Vinculum anteriorly and posteriorly concave. Tegumen rounded, forming obtuse pseuduncus; uncus band-shaped, with indistinct central process, with pair of setae; gnathos with long, pointed central element. Valva at base with long inner process, subterminally with inner process on dorsal surface, apex rounded. Transtilla without transverse bar. Aedeagus with ventral carinae ending in forked lobes, tightly fused to ventral process; pair of lateral, pointed carinae present; vesica with numerous small cornuti and one large, serrate cornutus.

Female genitalia (Fig. 45). T8 with few scales and setae only. Anal papillae with 19-21 setae. Vestibulum with elaborate sclerotisations, partly serrate and densely covered with pectinations. Total bursa length $c a$. $765 \mu \mathrm{m}$. Corpus bursae elongate, without pectinations, with very narrow and long reticulate signa (length $c a$. $420-450 \mu \mathrm{m})$, margins crenate. Ductus spermathecae with 2 convolutions and long and conspicuous vesicle.

Final instar larva. Head capsule (Fig. 84) ca. 355-390 $\mu$ m wide. Mandibular cusps blunt. A9 with 2 pairs of setae. Integument covered with extremely short microtrichia (ca $1 \mu \mathrm{m}$ long), absent on A9 and 10. Otherwise as group description.

Biology. Hostplants Platanus orientalis and planted P. hybrida.

Egg on leaf underside, usually against a vein. Leafmine (Figs. 102, 105) a long gallery with contorted frass, or partly linear frass, or with linear frass throughout, very variable; frass greenish when fresh, later turning brown; exit hole on upper surface. Confusion with other species is not possible, the only other European leafminer on Platanus is Phyllonorycter platani (Staudinger, 1870), which makes tentiform mines, starting with irregular galleries in the epidermis. Mines with thin frass throughout were sometimes considered to belong to a separate species (Hering 1957), but in fact belong to A. platani as well.

Life-history: probably bivoltine, with adults in May-June and again in July-August, larvae are found in June and September to November.

Distribution (Fig. 122). Widespread in southern Europe and western Asia, from Portugal to Iran. It had spread westwards from its original occurrence with Platanus orientalis in the Balkans before 1930, since it was found in western France and Switzerland around that time (Müller-Rutz 1934a; van Nieukerken et al. 2006). Surprisingly this species has not undergone a similar spread as have several Gracillariidae leafminers, such as the Platanus-feeding Phyllonorycter platani (see Sefrová 2001). The northernmost locality for A. pla- 
tani has for more than 70 years been the region of Paris, where it is still common. A supposed record for The Netherlands was based on a misidentification (Kuchlein 2002; Kuchlein et al. 2003). New records for Menorca, Bulgaria, Cyprus and Turkey.

Remarks. In the original description, Müller-Rutz described and illustrated the male wings as female and vice-versa.

Material examined. 19 ${ }^{x}, 18$, 9 larvae. CYPRUS: 1 larva on slide, Troodos, 1200 m, 28.ix.1990, B.

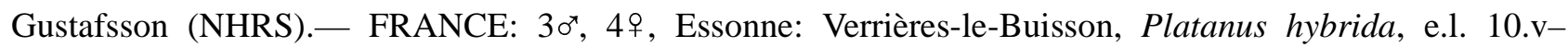
12.vi.1960, R. Buvat (MNHN); 2o`, Hérault: Montpellier, 7.vii.1991, P. hybrida, e.1. 8-31.vii.1991, A. \& Z. Laštůvka (AL); Var: 3 larvae on slide, Roquebrune-sur-Argens, 28.vii.1979, P. hybrida, J.W. Schoorl (RMNH). - GREECE: 1 larva on slide, Attika, Athínai: ancient Agora, 30.ix.1980, P. orientalis, S.B.J. Menken \& E.J. van Nieukerken (RMNH); 2 larvae on slide, Evvoia, Dhírfis Oros, S. slopes, 12.ix.1980, P. orientalis, Menken \& van Nieukerken (RMNH); 10`, Lakonia: 7 km SW Monemvasia, 18.vii.1980, G. Christensen (ZMUC); 2 larvae on slide, Samos, [Näktergalsdalen= valley of nightingales], 5.x.1994, B. Gustafsson

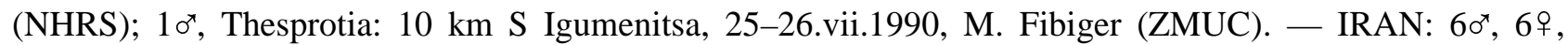

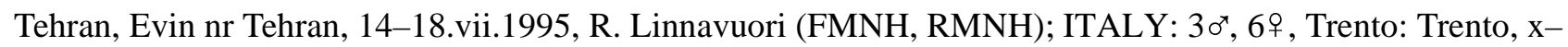

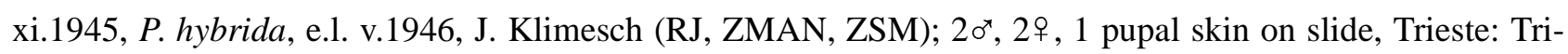
este, P. hybrida, e.l. 1.v.1934-31.v.1934, J. Klimesch (RMNH, ZSM). — TURKEY: 1o`, prov. Antalya, Alanya, Mahmutlar, Ruine, 17.v.2005, W. Mey (ZMHB).

Leafmines on Platanus hybrida: CROATIA: Rijeka, in town, 15.x.1983. ITALY: Latium: Roma, Villa Borghese, Zoological garden, 15.ix.2005; Latium: San Severa, 17.ix.2005. For material of many mines from France, Portugal and Spain see van Nieukerken et al. 2004a, 2006. Leafmines on Platanus orientalis: CYPRUS: Troodos, B. Gustafsson (NHRS). GREECE: Attika: Athínai: ancient Agora; Evritanía: Frangísta, 600m, 20.ix.1980; Mt Timfristós, S, slopes above Karpenision, 1300m, 19.ix.1980; Evvoia: 2 km W Prokopion, 13.ix.1980; Dhírfis Oros, S. slopes, 900m, 12.ix.1980; Steni Dhirfios, in village, 400m, 10.ix.1980; Fthiotis: Oíti Oros, N. slope 7 km SW Ipáti, 1400m, 15.ix.1980; Fókis: halfway Lílaia and Polídhroson, river valley, 400m, 28.ix.1980; Ioánnina: Voutonási, valley, 700m, 25.ix.1980 (collected by E. J. van Nieukerken and co-workers, unless noted differently, all in RMNH).

Additional records. BULGARIA: Kresna, 21.x.2004, vacated mines Platanus orientalis, observed, J. Buszko. - GREECE: 1 vacated mine, Lesvos: Parakila, 30.vi.2003, M. Hull (MH); 4o , 3 우, Pieria, Leptokaria, 23.vi.1997, A. \& Z. Laštůvka (AL). — SPAIN: Baleares, Menorca, 28.x.2006, vacated mines, R. Edmunds.

\section{Acalyptris minimella (Rebel)}

(Figs. 3, 18, 23, 24, 46, 61, 63, 72, 73, 85, 100, 101, 103, 106, 107, 123)

Trifurcula minimella Rebel, 1926: (110). Lectotype ơ (here designated): CROATIA: Sucurac D.[almatia], 5.vi.[19]24, P. Novak, Genitalia slide MV 13119 (NMW) [examined]

Weberina lentiscella Groschke, 1944: 117. Syntypes, 20, 19: ITALY. Sicily, Taormina, iv. 1942, iii-iv.1943, larvae on Pistacia lentiscus, e.l. 19.v.1942 \& 16-22.v.1943, Groschke [types presumed lost]. Neotype male (designated by Klimesch 1978: 257), SPAIN: Baleares, Mallorca, Palma Nova ex. 1. 26.vi.1968, mine in Pistacia lentiscus, 10.iii.1968, Zucht no 972, J. Klimesch (ZSMG) [not examined] (synonymized by Klimesch 1978)

Nepticula minimella; Klimesch 1953: 162 [new combination]

Trifurcula (Weberina) minimella; Klimesch 1978: 256, figs. 37 [partim, status of species; type studied]

Trifurcula (Niepeltia) minimella; Gustafsson 1981: 463 [larval description, partim, Balearic record].

Niepeltia minimella; Scoble 1980: 207 [redescription; new combination]

Acalyptris minimella; van Nieukerken 1986b: 141 [new combination, listed]; A. \& Z. Laštůvka 1993: 158 [listed]; Passos de Carvalho \& Corley 1995: 194 [Portugal]; van Nieukerken 1996: 24 [catalogued]; A. \& Z. Laštůvka 1997: 118 [description]; Corley et al. 2000: 250 [Portugal]; Lo Duca et al. 2002: 76, 97-106 [Sicily, parasites reared]; van Nieukerken et al. 2004a: 227 [Iberian Peninsula]; A. \& Z. Laštůvka 2005: 10 [Italy, Sicily]; van Nieukerken et al. 2006: 57 [France, Corsica]. 
Niepeltia lentiscella; Hering 1957: 781 [mine keys].

Weberina lentiscella; Klimesch 1978: 257, figs. 37 [synonymisation with minimella; neotype selection]

[Nepticula promissa?; Klimesch 1942: 396 [Croatia] partim, mine on Pistacia lentiscus, possible misidentification]

Diagnosis. Males of A. minimella and A. pistaciae resemble several pale Trifurcula s.l. species; males of these have a velvet patch on underside hindwing, and the Acalyptris species have hair-pencils on fore and hindwing. A. minimella is very similar to A. pistaciae, male externally recognised by presence of costal bristles and yellow spot on forewing underside. Male genitalia differ by basal valval lobe (medial in pistaciae) and V-shaped ventral process. In female genitalia by simpler vaginal sclerite and 3 convolutions in the ductus spermathecae as opposed to 2.5 in pistaciae.

Description. Male (Fig. 3). Forewing length 1.8-2.4 mm, wingspan 4.1-5.4 mm. Head: frontal tuft ochreous white to yellowish orange, occasionally mixed with fuscous; collar paler; scape and pedicel yellowish white. Antenna with ca. 32-39 segments. Thorax and forewing yellowish ochreous, irrorate with few pale brown scales; cilia white, cilia line not distinct. Underside at base with small central pale yellow spot, formed by lamellar androconial scales, remaining part darker; along dorsal margin at base a hair-pencil mixing with hindwing hair-pencil. Hindwing frenulum and costal bristles present; with field of yellowish hair and lamellar scales in basal 1/2, forming an indistinct hair-pencil; underside greyish white. Abdomen yellowish grey, with grey inconspicuous anal tufts.

Female. Forewing length 2.1-2.6 mm, wingspan 4.7-5.8 mm. Antenna with 28-33 segments. Thorax and forewing somewhat darker than in male; special scales absent; costal bristles present. Abdomen with wide ovipositor, no anal tufts.

Male genitalia (Figs. 23, 24, 63, 72, 73). Measurements: see Table 3. Vinculum anteriorly concave, ventral plate not large. Tegumen small, more or less triangular; uncus band-shaped, with indistinct central process, in lateral view split distally; gnathos with long narrow central element. Valva narrow, with large, more or less triangular, basal inner process; apex slightly pointed. Transtilla without transverse bar, sublateral process distinct. Aedeagus with bifurcate ventral carinae, tightly fused to V-shaped ventral process; a pair of curved dorsal carinae present; vesica with numerous small cornuti and one large cornutus.

Female genitalia (Fig. 46). T8 narrow, with produced lateral corners, two small lateral groups of scales and setae. Anal papillae conspicuous, with 17-21 setae. Apophyses straight, slender. Total bursa length $c a$. 720-880 $\mu \mathrm{m}$. Vestibulum with elaborate sclerotisations, more or less ring-shaped, inner margin partly serrate, with two processes. Ductus bursae covered with pectinations. Corpus bursae elongate, without pectinations; with narrow (2-3 cells wide) and long reticulate signa, margins crenate. Length shortest signum 270-320 $\mu \mathrm{m}$, longest 340-385 $\mu \mathrm{m}$. Ductus spermathecae with 3 convolutions and large vesicle.

Final instar larva (Figs. 85, 100, 101). Head capsule ca. 325-350 ìm wide. Mandibular cusps blunt. A9 with 3 pairs of setae. Integument covered with extremely short microtrichia (ca $1 \mu \mathrm{m}$ long) on all segments. Otherwise as group description.

Biology. Hostplant. Pistacia lentiscus (Anacardiaceae), only once recorded from P. terebinthus (Gibraltar: see material).

Egg on leaf upperside, usually close to a vein. Leafmine (Figs. 103, 106, 107) starting as an extremely narrow gallery, filled with frass, running a rather straight course, often following the margin of the leaflet and/ or the midrib; the thin part is more than half the total length of the mine; later mine widening gradually and becoming more contorted, often in zigzag course, with blackish or brownish frass coiled or in loose pellets, filling about two thirds of mine width; exit hole on leaf upperside. Leaflets with completed mines usually discolouring and easily falling off.

The mine may be confused with that of the sympatric Simplimorpha promissa (Staudinger, 1870), which tends to be more common on Pistacia terebinthus. Mines of S. promissa can be recognised by the absence of a very long, thin, initial mine; tenanted mines are easily separated by the green coloration of S. promissa.

Distribution (Fig. 123). Widespread and common in Western Mediterranean, usually not far from the sea: 
Portugal, Spain, France, Italy, Croatia, Morocco, Algeria, Tunisia, including the islands Ibiza, Mallorca, Corsica, Sardinia and Sicily. New records for Morocco, Algeria, and Tunisia.

Remarks. Rebel described Trifurcula minimella on the basis of an unspecified number of specimens from four dates, of which three males are still present in the Vienna Museum, all labelled as type. Two of these are the Acalyptris species, one is a Stigmella dorsiguttella (Johansson, 1971). I here select one of the Acalyptris specimens as lectotype (data above) in order to restrict the name to its current usage.

According to Klimesch (1978) the types of Weberia lentiscella Groschke are lost. However, there is still a possibility that syntypes are present amongst Groschke's material in Stuttgart. The fact that the material is only labelled with numbers would not necessarily prevent the location of the types. By deduction it has also been possible to select a lectotype of Nepticula caradjai Groschke, 1944 from this material (van Nieukerken 1985). The neotype selection by Klimesch may thus have been superfluous.

Material examined. 29 ${ }^{\star}, 24 ㅇ, 19$ larvae. ALGERIA: $3 ㅇ, 4$ larvae on slide: $11 \mathrm{~km}$ ESE Morris, 26.iv.1980, leafmines, Pistacia lentiscus, e.l. 29.v.1980, van Nieukerken \& Bryan (RMNH). - CROATIA: $10^{\star}$ (paralectotype): Sucurac Dalmatia, vii.1923, Novak, slide MV13120 (NMW). - FRANCE, CORSICA:

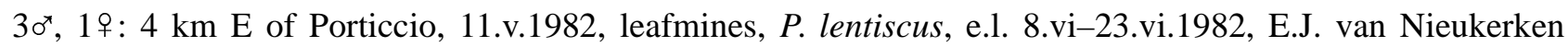
(RMNH, VPU). - GIBRALTAR: 1웅 Gibraltar, P. terebinthus, e.l. 30.v.1903, Walsingham (BMNH). -

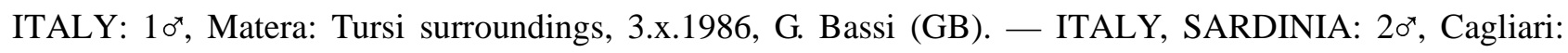
Monte Arcosu, Perdu Melis, 320 m, 25.vi.2004, P. Triberti \& G. Baldizzone; 20, 2우, Cagliari: Monte Arcosu, Sa Canna, 150 m, 22.vi, 3.vii.2004, P. Triberti \& G. Baldizzone, 10, Cagliari: Monte Arcosu, Sa Tragu, 29.vi.2004, P. Triberti \& G. Baldizzone; 30, Nuoro: $10 \mathrm{~km} \mathrm{~W}$ Dorgali, 26.vii.1999, P. Triberti (PT, RMNH); 30 , 2ㅇ: F. Cedrino, 5 km SW Galtelli, 16.vii.1999, P. Triberti (PT, RMNH). — ITALY, SICILY: 1우, Cinisi,

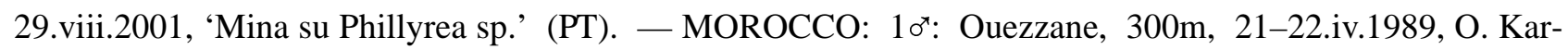
sholt (ZMUC). - PORTUGAL: 1ㅇ, Algarve: Cabo de Sao Vicente, 8.i.1987, leafmines, P. lentiscus, e.1. E.J. van Nieukerken (RMNH); 2 \% : Serra de Monte Figo, 24.v.2001, M.F.V. Corley (MC, RMNH). — SPAIN: 30', 4우, 7 larvae on slide: Baleares: Ibiza, San Antonio, 27.iv-7.v.1975, Pistacia, e.1. 29.v-9.vi.1975, B. Gustafsson (NHRS); 10, Mallorca: Es Comú, S. Albufeira, 20.x.2004, M.J. Honey (BMNH); 1 larva on slide, Mallorca, Magaluf, 2.v.1975, B. Gustafsson (NHRS); 1o`: Mallorca, Paguera, 1-10.v.1970, J. Klimesch (ZSM); $50^{\star}, 5$ ㅇ: Mallorca, Palma Nova, 10.iii.1968, P. lentiscus, e.l. 16.iv-23.ix.1968, J. Klimesch (ZSM); 1우 Málaga: Benahavis, 300m, 6.ii.1984, leafmines, P. lentiscus, e.1. 19.v.-21.v.1984, E.J. van Nieukerken $(\mathrm{RMNH}) ; 10^{\rtimes}$ : Camino de Ojen, 150m, 20.vi.1983, E. Traugott-Olsen (ZMUC); 1 ㅇ, 1 larva on slide: Marbella, 2 km N, 28.iii.1979, leafmines, P. lentiscus, e.1. 6.vi.1979, E.J. van Nieukerken (RMNH); 10`: Marbella, Casa y Campo, 100m, 5.x.1982, E. Traugott-Olsen (ZMUC). - TUNISIA: 10`: Ain Draham area, 5-18.v.1988, O. Karsholt (ZMUC); 2 larvae on slide, 11 km S Aïn Draham, Oued es Zaouïa, P. lentiscus, 6.v.1980, van Nieukerken \& Bryan (RMNH); 4 larvae on slide, 4 km W Tabarka, 0-140m, P. lentiscus, 25.iv.1980, van Nieukerken \& Bryan (RMNH).

Leafmines on Pistacia lentiscus: ALGERIA: 11 km ESE Morris, 26.iv.1980, van Nieukerken \& Bryan. ITALY, SICILIA: Catania: $3 \mathrm{~km}$ W of San Pietro, 27.iii.1981. - TUNISIA: $10 \mathrm{~km}$ W Jebel Abiod, 80m, 7.v.1980; 11 km S Aïn Draham, Oued es Zaouïa, 6.v.1980; 4 km W Tabarka, 0-140m, 25.iv.1980; Cap Bon, $12 \mathrm{~km}$ NE Soliman, 22.iv.1980. Mines collected by E. J. van Nieukerken and coworkers, in RMNH. The many records of mines from Corsica, Portugal and Spain cited in van Nieukerken et al. (2004a; 2006) are not repeated here.

Additional records. CROATIA: 60x, 4우, Dalmacia, Orasac, 15.vii.2000, 20.viii.2001, Pistacia lentiscus, A. \& Z. Laštůvka (AL). 
Acalyptris pistaciae sp. $\mathbf{n}$.

(Figs. 4, 25-27, 47, 64, 74, 75, 86, 96, 97, 104, 108-110, 124)

Niepeltia near minimella; van Nieukerken 1982: 19 [Greece]

Acalyptris sp. n.; van Nieukerken 1986b: 141 [listed]

Misidentifications:

Trifurcula (Weberina) minimella; Klimesch 1978: 256, figs. 36, 38-39 [partim, Greek records]

Trifurcula (Niepeltia) minimella; Gustafsson 1981: 463 [partim, Cyprus records, larval description]; Arenberger \& Wimmer 1996: 211 [Cyprus].

Nepticula promissa; Amsel \& Hering 1931: 137 [mines, Israel, possibly this species]

Type material. Holotype ơ: GREECE: Fókis, 3 km E Dhelfoi (UTM: 34S FH3460), 700m, 27.ix.1980, leafmines, Pistacia terebinthus, e.l. 14.iv-11.v.1981, Menken \& van Nieukerken, Genitalia slide EvN1269 (RMNH-INS no 21269) (RMNH). - Paratypes: 33 ${ }^{x}, 15$ 15, 5 larvae. CYPRUS: $10^{x}$ : Agios Theodores (27),

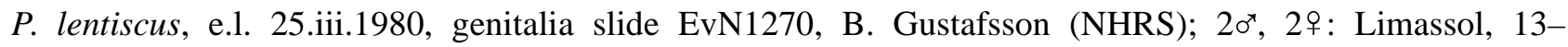

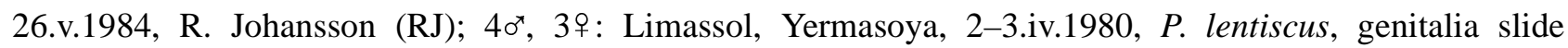
EvN1268, B. Gustafsson (NHRS, RMNH); 1요 Platies, 27.iii.1980, P. lentiscus, B. Gustafsson (NHRS). GREECE: 10 $0^{\star}$, Argolis, Porto Kheli, Cap d'Or (Porto Heli, Cape d'Or), 4-9.vi.1980, L. Kohonen, genitalia slide EvN2684 (RMNH); 10, Arkadia: $10 \mathrm{~km} \mathrm{~S}$ of Leonídhion, 800m, 10.vii.1991, R.T.A. Schouten

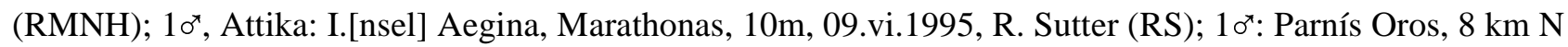
Dhekélia, 700m, 29.ix.1980, leafmines, P. terebinthus, e.1. 22.iv.1981, genitalia slide EvN0487, Menken \& van Nieukerken; 1 larva on slide, Evvoia: 2 km SE Gouvés, 200m, 14.ix.1980, Menken \& van Nieukerken; 40 $0^{x}$ 3 : Fokis: same data as holotype, genitalia and wing slides EvN0959 \& 1266; 3 larvae on slides, same data as holotype, slide numbers RMNH 12395-12397 (RMNH); 10, Lakonia: $5 \mathrm{~km} \mathrm{~S}$ Monemvasia, 1.viii.1978, G. Christensen; 1ㅇ: 5 km S Monemvasia, 30m, 1-8.vii.1982, B. Skule \& S. Langemark (ZMUC). — GREECE, CRETE: 20`, Iráklion: 2 km S Khersonisos, road to Kastelion, 200 m, 14.vi.1998, H. W. van der Wolf (RMNH); 6o 1우, Lasíthi: Makrygialos, 12, 13, 14, 21.vi.1988, R. Johansson, genitalia slide RJ1733;

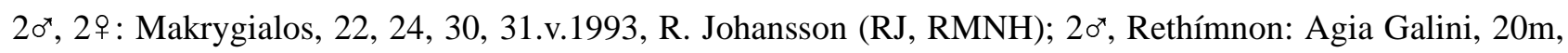
19-20.v.1994, R. Sutter (RS). — GREECE, KOS: 4ox, Kos, Asfendiou, 6, 12.x.1988, R. Johansson, genitalia slide RJ1732 (RMNH, RJ). — GREECE, RÓDHOS: 1ㅇ, Ródhos: Jalyssos, 5 km SW Rhódos, 10.viii.1982 (ZMUC); 10`, 1우: Rodini, 22.ix.1972, P. lentiscus, e.1. 27.x-7.xi.1972, J. Klimesch, Genitalia slides KL1311, 1312 (ZSM). - TURKEY: 1 larva on slide, Antalya: Selimiye (ancient Side), near beach, 4.iii.2005, P. lentiscus, E.J. van Nieukerken, slide number RMNH11892 (RMNH).

Other material. Leafmines on Pistacia lentiscus : CYPRUS: Limassol, 10-25.v.1984, R. Johansson; Limassol, Yermasoyia, 2-3.iv.1980, B. Gustafsson; Plates, 27.iii.1980, B. Gustafsson. GREECE: Evvoia, Néa Artáki, 100m, 11.ix.1980. TURKEY: Antalya: Phaselis ruins, 3km NE Tekirova, 4.iii.2005; Antalya: Selimiye (ancient Side), near beach, 4.iii.2005. - Leafmines on P. terebinthus: GREECE: Argolis: $1.5 \mathrm{~km}$ NE Toló: ancient Asine, rocky headland, 50m, 7.ii.1990; Attika: Athínai: ancient Agora, 30.ix.1980; Parnís Oros, 8 km N Dhekélia, 700m, 10+29.ix.1980; Evvoia: 2 km SE Gouvés, 200m, 14.ix.1980; Fókis: 3 km E Dhelfoi, 700m, 27.ix.1980; Kerkyra: Pandokraton, 24.x.1987, J.H. Donner; Khios: Anavatos, 3.viii.1999, C. van den Berg; Vrondadhes, Daskalopetra, 7.viii.1999, C. van den Berg; Lakonia: Mistrás, 400m, 21.vi.984. (collected by E. J. van Nieukerken and coworkers, unless noted differently, all (at least partly) in RMNH).

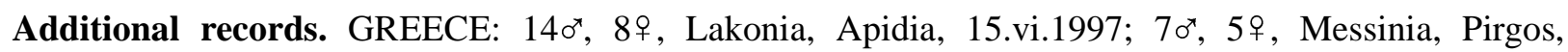
13.vi.1998; 80^, 7우, Préveza, Thesprotiko, 11.vi.1997, A. \& Z. Laštůvka (AL).

Diagnosis. A. pistaciae is very similar to A. minimella, male differs by the absence of a central yellow spot on the forewing underside and absence of costal bristles; also the hairpencil is more distinct in pistaciae. Male genitalia characterized by medial toothed lobe of valva in contrast to the basal lobe in minimella, and by the U-shaped ventral process; the female genitalia are distinguished by the elaborate vaginal sclerotisation with three processes and the $2 \frac{1}{2}$ convolutions in the ductus spermathecae ( 3 in minimella). 
Description. Male (Fig. 4). Forewing length 1.8-2.4 mm, wingspan 4.0-5.3 mm. Frontal tuft ochreous white to yellowish white, occasionally with few fuscous scales. Scape white, antenna with 27-33 segments. Thorax and forewing ochreous, irrorate with few light brown scales, cilia white, cilia line indistinct; underside without yellow spot; basalmost dorsal cilia forming long hairpencil. Hindwing grey, costal bristles absent; distinct, yellowish brown hairpencil arising near frenulum (Figs. 96, 97); mixing with forewing hairpencil; underside yellow basally. Abdomen yellow with darker middorsal line; anal tufts grey.

Female. Forewing length 1.6-2.2 mm, wingspan 3.7-4.9 mm. Antenna with 20-30 segments. Hairpencils absent. Abdomen dorsally brown, abdominal tip broadly rounded.

Male genitalia (Figs. 25-27, 64, 74, 75). Measurements: see Table 3. Vinculum anteriorly concave, ventral plate not large. Tegumen small, triangular; uncus band-shaped, with indistinct central process, in lateral view split distally; gnathos with long narrow central element. Valva narrow, with medial inner lobe, with variably toothed margin and a smaller lobe dorsally, hidden by ventral lobe. Transtilla without transverse bar, sublateral process distinct. Aedeagus with bifurcate ventral carina or with 4-5 lobes, ventrally tightly fused to Ushaped ventral process; a pair of curved dorsal carinae present; vesica with large curved cornutus and basal sclerotisation associated with cathrema; many smaller cornuti.

Female genitalia (Fig. 47). T8 narrow, with produced lateral corners, two small groups of scales and some setae. Anal papillae conspicuous, with 15-20 setae; apophyses straight, of approximately same length. Total length of bursa $c a$. 530-760 $\mu \mathrm{m}$. Vestibulum with elaborate sclerotisations, more or less forming ring and three apophyses, partly serrate. Ductus bursae with many pectinations; corpus bursae without pectinations; with reticulate signa, shortest $c a$. 285-325 $\mu \mathrm{m}$, longest 310-420 $\mu \mathrm{m}$ long, margin crenate and narrow, 2-3 cells wide. Ductus spermathecae with $2 \frac{1}{2}$ convolutions.

Final instar larva (Fig. 86). Head capsule ca. 315-380 $\mu$ m wide. Mandibular cusps blunt. A9 with 3 pairs of setae. Integument covered with extremely short microtrichia (ca $1 \mu \mathrm{m}$ long) on all segments. No obvious differences with A. minimella.

Biology. Host plants: Pistacia terebinthus and P. lentiscus (Anacardiaceae), frequently found on both hosts.

Egg on leaf upperside, usually close to a vein. Leafmine (Figs. 104, 108-110) on P. lentiscus indistinguishable from that of minimella, starting as an extremely narrow gallery filled with frass; the thin part is more than half the total length of the mine; later, mine widening gradually and becoming more contorted, less often in a zigzag course than minimella, with blackish or brownish frass coiled or in loose pellets filling about two thirds of mine width; exit hole on leaf upperside. Mines in the thinner leaves of P. terebinthus a narrow gallery throughout, gradually widening, frass leaving clear margins, occasionally frass line becoming wider. The mine on both hosts may be confused with that of Simplimorpha promissa; see under A. minimella.

Distribution (Fig. 124). Eastern Mediterranean region: Widespread in Greece, including its islands, and in Cyprus and Turkey. To be expected in Syria and Lebanon. Mines collected in Israel by Amsel \& Hering (1931) and identified as Simplimorpha promissa (Staudinger) are tentatively regarded to be this species, although no adults were seen. The distribution of $A$. pistaciae is apparently completely vicariant with that of A. minimella, the contact zone (if any) may be found between Montenegro and northern Greece.

Etymology. A noun in genitive case, named after the host genus Pistacia.

Remarks. When treating the Pistacia-feeding A. minimella (Rebel), Klimesch (1978) recognized two forms, but considered this as intraspecific variation. It has now become clear that they are two different allopatric species, as was assumed before (Scoble 1980; van Nieukerken 1986b).

In Iran nepticulid mines have been commonly found on Pistacia khinjuk and the cultivated Pistachio nut $P$. vera (Reza Mehrnejad, pers. comm.), and a cocoon with a dried pupa has been examined by me. The material was insufficient for further identification, but it almost certainly represents an Acalyptris species; also photographs of leafmines studied by me resemble those of A. pistaciae. It is thus possible that A. pistaciae occurs in Iran as well, or another species. The previous records for Simplimorpha promissa from Iran (Davatchi 1958, Mehrnejad 2001) also are likely to belong to this Acalyptris. 


\section{Acalyptris loranthella (Klimesch)}

(Figs. 5, 6, 8, 22, 28, 48, 65, 87, 91, 111, 125)

Nepticula loranthella Klimesch, 1937: 34. Syntypes: HUNGARY: Pecs, Mitte Juni [vi.]1936, mines on Loranthus europaeus, e.1. 3-6.vii.1936, J. Klimesch (ZSM) [not examined]

Stigmella loranthella; Klimesch 1948: 78-80, fig. 61, 62; Hering 1957: 631 [mine keys]; Drăghia 1968: 860 [Rumania]; Kasy 1969: 2 [Austria]; Popescu-Gorj et al. 1972: 182 [Rumania]; Hansson 1985: 92 [parasite reared, Greece, Hungary].

"Stigmella" loranthella; van Nieukerken 1982: 19 [Greece].

Weberina loranthella; Szőcs 1978: 268 [new combination, Hungary]

Niepeltia loranthella; van Achterberg 1983: 30 [parasite records]

Acalyptris loranthella; van Nieukerken 1986b: 141 [new combination, listed]; A. \& Z. Laštůvka 1990: 187 [Czechoslovakia]; A. \& Z. Laštůvka 1993: 158; van Nieukerken 1996: 24 [catalogued]; A. \& Z. Laštůvka 1997: 116 [description; Italy]; Tokár et al. 2002: 1 [Slovakia]; A. \& Z. Laštůvka 2005: 10 [Sicily].

Diagnosis. Male with distinctive black forewing and white basal patch, scape with black distal part and black androconia on hindwing. Female resembles A. minimella and A. pistaciae, but has a dark frontal tuft and darker forewing. In genitalia it differs from the previous three species by valval lobe, which is simply triangular and not as elaborate as in the other species. In the female genitalia A. loranthella is recognised by wider signa than in previous three species, about 7 cells wide.

Description. Male (Figs. 5, 8). Forewing length 1.8-2.4 mm, wingspan 3.7-5.5 mm. Head: frontal tuft fuscous; collar greyish, paler; scape white, distal 1/3 to more than $1 / 2$ black. Antenna grey, with 30-39 segments. Thorax black, with few scattered greyish white scales; forewing covered with black scales, white elongate patch in costal half near base, not reaching costa and variable amount of pale grey scales around this patch, and in dorsal half forming elongate, almost linear, patch; terminal 1/5 white with grey, cilia silvery white. Underside with black scaling at base and in narrow strips along costa and dorsum, rest of underside devoid of scales, white patch shining though, black scaling appearing grey. Hindwing silvery white, frenulum and costal bristles present; patch of black scales covering basal 1/6-1/5; underside similar, with similar black patch. Abdomen silvery grey, with white inconspicuous anal tufts.

Female (Fig. 6). Forewing length 2.1-2.5 mm, wingspan 4.5-5.5 mm. Antenna with 33-36 segments. Frontal tuft fuscous to brown, collar ditto. Scape yellowish white, no black scaling. Thorax and forewing yellowish brown to ochreous, irrorate with dark brown scales; cilia silvery white; special scales absent; costal bristles present, hindwing silvery white. Abdomen grey ochreous.

Male genitalia (Figs. 22, 28, 65). Measurements: see Table 3. Vinculum anteriorly concave, ventral plate rather long. Tegumen small, more or less triangular; uncus band-shaped, with indistinct central process, in lateral view split distally; gnathos with long narrow central element. Valva triangular because of triangular basal inner process, not clearly separated from valva; apex slightly pointed. Transtilla without transverse bar, sublateral process short. Aedeagus with bifurcate pointed carinae, ventrally tightly fused to ventral process; pair of curved dorsal carinae present; vesica with numerous small cornuti and several larger cornuti.

Female genitalia (Fig. 48). T8 narrow, two small lateral groups of scales and setae. Anal papillae conspicuous, with 23-27 setae. Apophyses straight, slender. Total bursa length $c a$. 730-800 $\mu \mathrm{m}$. Vestibulum with elaborate sclerotisations. Ductus bursae covered with pectinations. Corpus bursae elongate, without pectinations; with wide (up to 7 cells wide) and long (ca. 305-345 $\mu \mathrm{m}$ ) reticulate signa, margins crenate. Ductus spermathecae with ca. 2 inconspicuous convolutions and small vesicle.

Final instar larva (Fig. 87). Head capsule ca. 290-315 $\mu$ m wide. Mandibular cusps pointed. A9 with 3 pairs of setae. Integument covered with rather long microtrichia (ca. 7-16 $\mu \mathrm{m}$ long) on all segments except A10. Otherwise as group description.

Biology. Hostplant. Loranthus europaeus (Loranthaceae), an obligate parasite growing on branches of Quercus species and Castanea sativa. 
Egg on either side of leaf, mine developing on the side where the egg is deposited. Leafmine (Fig. 111) a gallery throughout, often starting as a spiral mine, the coils amalgamating to form a small blotch, gallery often crossing earlier course, thereby forming a false blotch. Frass brownish, starting as broken linear tract, later slightly coiled or dispersed, filling one third to one half of mine width; exit hole on the same side as the egg and the mine.

Distribution (Fig. 125). Occurs with host in South eastern Europe: Czech Republic, Slovakia, eastern Austria, Hungary, Italy, Sicily, Rumania and Greece. To be expected throughout Balkan peninsula and in Turkey.

Material examined. $25 \circ^{x}, 23$ 우, 5 larvae. CZECH REPUBLIC: $10^{x}, 1$ 우, Moravia, $3 \mathrm{~km}$ NE Mikulov, Milovicsky Les, 3.x.1992, leafmines, Loranthus europaeus, e.l. 31.iii.1993, E.J. van Nieukerken (RMNH); 1우: Lednice, castle gardens, 3.x.1992, leafmines, L. europaeus, e.1. 31.iii.1993, E.J. van Nieukerken

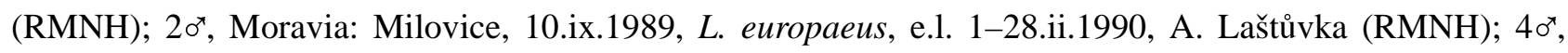
11우, Milovice, 9.ix.1994, L. europaeus, e.1. iii.1995, R. Johansson (RJ); $50^{\star}, 1$ 우, Moravia S: Milovice, Kralici Vrch, W. slope, 6 km NE Mikulov, 200m, 9.ix.1994, open Quercus pubescens stands; leafmines, L. europaeus, e.l. 26.iv -8.v.1995, E.J. van Nieukerken (RMNH). - GREECE: 3ox 29,1 larva on slide, 1 pupal skin on slide, Evvoia: Dhírfis Oros 2 km NE Steni, 500m, 11.ix.1980, leafmines, L. europaeus, e.1. 18.iii-10.iv.1981, S.B.J. Menken \& E.J. van Nieukerken (RMNH); 60, 5 ㅇ, 1 pupal skin on slide, Dhírfis Oros, S. slopes, 800m, 12.ix.1980, leafmines, L. europaeus, e.1. 13.iii-13.iv.1981, S.B.J. Menken \& E.J. van Nieukerken (RMNH); 4 larvae on slide, Fthiotis: 1-2 km W Timfristós village, 1000m, 20.ix.1980, S.B.J. Menken \& E.J. van Nieukerken (RMNH). — SLOVAKIA: $10^{x}$ : Vinianska Stran, 25.vi.1994, Z. Tokár (RJ); 30, 2우, Slov. Kras.: ca 2.5 km N Plesivec: Hôrka, 300m, 8.x.1992, leaf-mines, L. europaeus, e.l. 23.v.1993, E.J. van Nieukerken (RMNH).

Leafmines. On Loranthus europaeus: AUSTRIA: Niederösterreich: Gumpoldskirchen: Glaslauterriegel, 300-340m, 1.x.1983. - CZECH REPUBLIC: Moravia, 3 km NE Mikulov, Milovicsky Les, 3.x.1992; Milovice, Kralici Vrch, W. slope, 6 km NE Mikulov, 200m, 9.ix.1994. - GREECE: Evritanía: 3 km NE Frangísta, 1000m, 20.ix.1980; Evvoia: Dhírfis Oros 2 km NE Steni, 500m, 11.ix.1980; Dhírfis Oros, S. slopes, 800m, 12.ix.1980; Fthiotis: 1-2 km W Timfristós village, 1000m, 20.ix.1980. — SLOVAKIA: Slov. Kras.: ca 2.5 km N Plesivec: Hôrka, 300m, 8.x.1992 (mines all collected by E. J. van Nieukerken and coworkers, in RMNH).

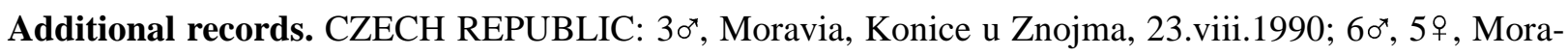
via, Mikulov, e.l. iii.1991; 160 , 13우, Moravia, Sedlec u Mikulova, e.l.iv.2002; $30^{\star}$, 2우, Moravia, Vranovice, 5.vii.1999. - GREECE: 1우 Akhaia, Kalavrita, 20.vi.1997. — ITALY: Potenza, Albáno di Lucania 22.vi.1995; all A. \& Z. Laštůvka (AL).

\section{The Acalyptris staticis group}

This species group is established here for the Plumbaginaceae-feeding species and A. pyrenaica. There are no representatives known outside Europe and the Mediterranean region.

These species differ considerably from most other Acalyptris, the most important shared character being the generic apomorphy: the venation with the closed cell in forewing shifted towards base. In contrast to most other Acalyptris, the transverse bar of the transtilla is present as a sclerotized bar (the plesiomorphic condition). The male genitalia are otherwise rather uniform, the vesica being reduced, without any structure, and the aedeagus relatively short. The female genitalia are characterised by a reduced corpus bursae and the absence of sclerotisations, a condition very similar to that in the genus Parafomoria (see van Nieukerken 1983). The reduced bursa and absence of cornuti in the aedeagus are probably related characters. Externally the males lack androconial scaling. 
Adult. Head: collar small, comprising hair-scales. Forewing pale, often with fascia, or uniform; cilia-line normally present, often indistinct. Forewing underside without subdorsal retinaculum. Hindwing with costal bristles in male and female. Venation as in platani group (Fig. 19). No androconial scales present. Male with anal tufts on T8, female sometimes with tufts on T7. Sternite 2 with $2 \mathrm{~A}$ broadly triangular, almost as wide as long (Fig. 60).

Male genitalia. Vinculum invisibly fused with tegumen, forming complete ring; ventral plate rounded, with posterior process joined to ventral carina. Tegumen forming variably shaped prominent pseuduncus. Uncus with single medial process. Gnathos with single medial element. Valva elongate, with a dense group of thick setae terminally on dorsal face. Transtilla with transverse bar present. Aedeagus with bifurcate ventral carina and a pair of curved lateral carinae, curving dorsad; vesica without any structure, no cornuti, but also no cathrema (the striate thickening around base of ductus ejaculatorius). Aedeagus relatively short, ratio aedeagus: capsule 0.6 to 0.8 (Table 3 ).

Female genitalia. Terminalia blunt. T8 single narrow plate, with single setae or groups of setae. T9 comprising distinct pair of anal papillae, often with many setae. S7 in some species also with dense group of long setae. Corpus bursae hardly developed, without any sclerotisation or signa; as such no measurements of bursa presented. Ductus spermathecae inconspicuous, with ca 2-3 coils.

Immature stages. Number of instars unknown. Final instar larva. Body yellow. Headcapsule (Fig. 88) wider than long. Frontoclypeus narrowing posteriorly, slightly stirrup-shaped. Anterior tentorial arms long, approximately twice as long as posterior arms. Labrum with medial and lateral setae. Labial palpi with two short segments and long apical seta. Antenna with sensilla not placed cross-wise (Van Nieukerken 1986a).

Prothorax with elongate, narrow, ventral sclerite and narrow paired dorsal sclerites. Pro-, meso- and metathorax respectively with 11, 7 and 7 pairs of setae. A1-8 with 6, A9 with 2 and A10 with 3 pairs of setae. Most setae relatively short, ventral setae longest. Reduction on meso- and metathorax affects the dorsal and lateral setae groups, SV1 and the three more ventral setae are all present. Anal rods almost straight, posteriorly ending in 3 pointed tips (Fig. 90). Spinosity of thorax and abdomen reduced, microspines scattered, hardly visible at a magnification of $400 \times$. Earlier instars not studied.

Pupa (studied from exuviae). Head: clypeus transverse; frons not completely detached at emergence, smooth; labial palpi slightly longer than maxillae. A2-8 dorsally with many relatively short spines, arranged in about 3-4 indistinct transverse rows per segment; cremaster comprising two widely separate spines (Fig. 92).

Biology. Hostplants. Five of the six known species feed on Plumbaginaceae, and four of these on Limonium species (Sea lavenders) along the sea coast. Only A. pyrenaica apparently is feeding on a different host family, because it was found in localities where this plant family is absent. Limonium is a very diverse plant genus in the Mediterranean region. Identification of Limonium is problematic and usually only possible when flowers are present. Previous records of Limonium vulgare as a host in the Adriatic and Aegean area (A. \& Z. Laštůvka 1997; Z. \& A. Laštůvka 1998) are possibly correct, but should be confirmed on flowering individuals because there are several very similar species.

Phylogenetically the Plumbaginaceae belong to the Caryophyllales, a basal clade in the Core Eudicots in the sense of the APG II classification (Bremer et al. 2003), and are considered sister to the Polygonaceae.

This group contains the only Nepticulidae found so far that have adapted to halophytic plants and live in a coastal habitat. The larvae make gallery mines, and the cocoons are spun on the leaves, probably as an adaptation to avoid lengthy submersion by sea water.

The mines of all Limonium-feeding species are very similar, usually starting with a more or less spirally arranged gallery, with a thin line of frass. Larva feeds in mine with ventral side uppermost. 
Stigmella staticis Walsingham, 1908: 1010. Lectotype $\sigma^{\pi}$ (here designated). SPAIN, CANARY ISLANDS: Tenerife: Pto. Orotava, Tenerife, 4.v.1907, Limonium pectinatum, e.1. 30.v.1907, Walsingham, no. 99202, Walsingham Collection 1910-425, Genitalia slide BM24095 (BMNH) [examined].

Stigmella staticis; Hering 1957: 618 [mine keys]; Klimesch 1977: 201-202, figs. 34-36 [redescription, genitalia]. Acalyptris staticis; van Nieukerken 1986b: 141 [new combination, listed] ; A. \& Z. Laštůvka 1993: 158 [listed].

Diagnosis. A. staticis is recognised by the uniform grey brown colour, and absence of any special scales. It slightly resembles Trifurcula species, but the lack of special scales separates the males easily. The male genitalia differ from other species in this group by the medially broadened valva, blunt uncus and bilobed posterior process of the vinculum. The female genitalia are similar to other species in this group but differ by the non hairy abdominal tip with just a few setae only.

Description. Male (Fig. 9). Forewing length 1.5-2.3 mm; wingspan 4.3-4.7 mm. Head: frontal tuft and collar yellowish orange; scape yellowish white mixed with brown scales, flagellum grey brown. Antenna with 38-40 segments. Thorax and forewing uniformly grey brown; cilia line indistinct, cilia grey. Hindwing and underside of both wings with same colour as forewing. Hindwing with costal bristles. Abdomen grey.

Female. Forewing length 1.9-2.3 mm, wingspan 4.0-5.0 mm. Antenna with 34-36 segments.

Male genitalia (Figs. 29-32, 67, 79-81). Measurements: see Table 3. Vinculum rounded ventral plate narrow, with bilobed ventral, posterior process; lateral arms expanded ventrally. Tegumen rounded, with few setae dorsally, Uncus ribbon-like, without central element, with prominent seta on either half. Gnathos with long spatulate central element. Valva (Fig. 67) widest in middle, inner margin forming approximately triangular lobe; tip rounded with many spine like setae dorsally; transtilla with curved transverse bar and long sublateral processes. Aedeagus (Figs. 30, 32, 80, 81) widest at tip, with two pairs of carinae: ventral carina bifurcate, pointed; lateral carina slightly curved dorsad. Vesica without any cornuti.

Female genitalia (Fig. 49). T8 narrow, indistinct, with few small setae and scales. T9 with two groups of 10-14 setae. Vestibulum and bursa flimsy, without sclerotisations, bursa hardly reaching beyond segment 7. Ductus spermathecae with 2-21/2 narrow convolutions.

Larva not examined.

Biology. Hostplant: Limonium pectinatum (=Statice pectinata), growing on rocky shores.

Egg on leaf underside. Leafmine (Figs. 118, 119) a gallery throughout, initially leaving parenchyma layers on both sides of leaves intact, appearing green, filled with frass; later a full-depth mine with narrow broken frass; mine contorted, usually following a curved zigzag track, with each turn touching previous turn.

Distribution (Fig. 126). Macaronesia: Canary Islands, only known from Tenerife. This species may be under threat, since many of the localities for its hostplant suffer from building activities for tourism. However, it is also possible that A. staticis may still be found along other coasts, such as other Canary Islands or that of Morocco.

Material examined. 8 $0^{x}, 10$ 우. SPAIN, CANARY ISLANDS: $20^{x}, 6$ 우 (Lecto- and paralectotypes): Tenerife: Pto. Orotava, Tenerife, 4.v.1907, Limonium pectinatum, e.1. 15-31.v.1907, Walsingham (BMNH); $10^{\star}$ : Puerto de la Cruz, 4.iv.1965, L. pectinatum, e.1. 28.iv.1965, J. Klimesch; 4o*, idem, 19.i.1969, e.1. mid ii.1969; 1ㅇ, idem, 3.iii.1969, e.1. 1.iv.1969; 10`, 3우: idem, 23.i.1973, e.l ii.1973 (ZSM, RMNH, RJ).

Leafmines on Limonium pectinatum. SPAIN, CANARY ISLANDS: Tenerife: Puerto de la Cruz, 26.iii.1965 + 18.i.1970, J. Klimesch (RMNH). 
Acalyptris limonii Z. \& A. Laštůvka, 1998: 314. Holotype ơ, GREECE: Arta, Salaora, ex larva 1996, Raupe 15.vi.1996, Limonium vulgare (AL) [not examined]

Diagnosis. A distinctly fasciate species, resembling A. lesbia. Female easily recognised by conspicuous anal tuft; A. limonii is much darker than A. maritima. Superficially resembling several Ectoedemia species. Male genitalia separated from the similar A. maritima and lesbia by narrow, pointed gnathos, uncus much longer than in maritima, about equal to lesbia. Female genitalia easily recognised from the other Limonium feeders by the tufts on T7 (sockets visible in preparations); the very hairy anal papillae and S7 are similar to A. lesbia and A. limoniastri. The latter also has similar male genitalia, but lacks the pointed uncus and has much larger male genitalia.

Description. Male (Fig. 11). Forewing length $1.9 \mathrm{~mm}$, wingspan 4.1-4.3 mm. Head: frontal tuft ochreous yellow, scape white with few ochreous scales. Antenna with 32 segments. Thorax and forewing white, covered with dark-tipped scales; dark tips half scale length; a narrow fascia at $2 / 3$, cilia white, cilia line present. Hindwing grey-white, dark costal bristles. Underside of forewing brownish, hindwing white; white hairpencil from underside near frenulum. Abdomen grey brown, with conspicuous yellowish white anal tufts.

Female (Fig. 12). Forewing length $2.3 \mathrm{~mm}$ (1), wingspan $4.8 \mathrm{~mm}$. Antenna with 32 segments. Abdominal tip with conspicuous yellow anal tufts, more or less paired.

Male genitalia (Figs. 39, 40, 43, 68). Measurements: see Table 3. Vinculum rounded with ventral plate narrow; lateral arms slightly expanded ventrally. Tegumen forming slightly produced pseuduncus with many setae dorsally. Uncus with long, pointed central element. Gnathos with a long narrow spatulate central element. Valva (Fig. 68) length not measured (not flat in slide), basally broadest; tip slightly produced inwards, with many thick setae dorsally; transtilla with almost straight transverse bar and short sublateral processes. Aedeagus (Figs. 40, 43) with ventral carina bifurcate, pointed; lateral carinae strongly curved dorsad. Vesica without any cornuti.

Female genitalia (Figs. 53, 54). Paired tufts of many scales on T7, no setae. T8 trapezoid, slightly indented mesially, with a small patch of few setae and scales. T9 with two groups of $c a 45-50$ setae. S7 also with many long setae near abdominal tip. Vestibulum and bursa flimsy, without sclerotisations, bursa hardly reaching beyond segment 7. Ductus spermathecae with 2 narrow convolutions.

Biology. Hostplant: Limonium cf. vulgare (see under group description), growing in salt marshes.

Egg on either side of leaf. Leafmine (Figs. 113, 114) a gallery throughout, starting much contorted, often spirally, later a full-depth mine with narrow broken frass, running more straight through leaf. Larval exit hole on leaf upperside. Mines indistinguishable from those of A. maritima or A. lesbia.

Cocoon white, usually spun on leaf underside.

Distribution (Fig. 127). Coasts of Adriatic, Ionian and Aegean Seas: recorded from Croatia (new record) and Greece.

Remarks. The relatively large bursa as illustrated in the original description has not been observed by me.

Material examined. 2 $\sigma^{\star}, 2 \circ$. GREECE: $2 \sigma^{\star}, 2 \circ$, paratypes, Arta: Salaora, 15.vi.1996, Limonium vulgare, e.1. vi.1996, A. Laštůvka (AL, RMNH,CVDB).

Leafmines on Limonium cf. vulgare.: CROATIA: Istra, Baska-Trget, 25.viii.1998, A. Laštůvka (RMNH). - GREECE: Arta: Salaora, 15.vi.1996, A. Laštůvka (RMNH) [mixed with inseparable mines of maritima]

Additional records. CROATIA: 10, Istra, Baska-Trget, 25.viii.1998, Limonium vulgare, e.p. ix. 1998. GREECE: $30^{x}$, Arkadia, Paralion Astros, e.1. vii.1998, A. Laštůvka (AL). 
Acalyptris maritima A. \& Z. Laštůvka, 1997: 119. Holotype ơ, GREECE: Arta, Salaora, 15.vi.1996, ex 1. VI.1996, A. Laštůvka, Limonium vulgare (AL) [not examined].

Diagnosis. A very pale species with fascia hardly visible, much paler than A. limonii or lesbia. Female slightly darker, fascia inconspicuous, separated from limonii by absence of anal tuft; A. lesbia female is darker and has a conspicuous fascia. Male genitalia as A. limonii and lesbia, separated by very wide triangular gnathos, uncus much shorter than in other two and aedeagus relatively short compared to lesbia. Female genitalia differ from A. lesbia and limonii by the sparse setae of the anal papillae and the absence of many setae on S7.

Description. Male (Fig. 13). Forewing length 2.3-2.6 mm, wingspan 5.0-5.5 mm. Head: frontal tuft and collar pale ochreous to ivory white; scape ivory white with few brown-tipped scales. Antenna with 32-36 segments. Thorax and forewing cream white, with brown-tipped scales (tips ca 1/4 of scale length), leaving narrow fascia at 2/3; cilia silvery white, cilia line present. Hindwing silvery white, with costal bristles. Underside of forewing darker yellowish brown, underside of hindwing yellowish brown, small white hairpencil arising near frenulum. Abdomen yellowish grey, anal tufts conspicuous, ochreous.

Female. Forewing length $2.1 \mathrm{~mm}$, wingspan $4.5 \mathrm{~mm}$. Antenna with 32 segments. Abdominal tip rounded, without anal tufts.

Male genitalia (Figs. 36, 37, 42, 69). Measurements: see Table 3. Vinculum anteriorly rounded, with ventral plate narrow, posteriorly with triangular ventral process; lateral arms slightly expanded ventrally. Tegumen forming slightly produced, rounded pseuduncus with many setae dorsally. Uncus with short, pointed central element, slightly curved posteriorly. Gnathos with broad triangular central element with cuspidate tip, lateral arms 'swollen'. Valva (Fig. 69) basally broadest; tip slightly produced inwards, with many thick setae dorsally; transtilla with very straight, long transverse bar and short sublateral processes. Aedeagus (Fig. 42) with ventral carina bifurcate, pointed; lateral carinae strongly curved dorsad. Vesica without cornuti.

Female genitalia (Figs. 55, 56,). No tufts on T7, no sockets visible. T8 trapezoid, slightly indented mesially, with few setae and scales. T9 in total with ca 26 setae. S7 with short setae only near abdominal tip. Vestibulum and bursa flimsy, without sclerotisations, bursa hardly reaching beyond segment 7 . Ductus spermathecae with 2 narrow convolutions.

Biology. Hostplant: Limonium cf. vulgare (see under group description), growing in salt marshes.

Egg on either side of leaf. Mine a full-depth mine, gallery throughout, starting much contorted, often spirally, later a full-depth mine with narrow broken, brown, frass, running more straight through leaf. Larval exit hole on leaf upperside. Mines not separable from those of A. limonii or A. lesbia.

Cocoon white, usually spun on leaf underside.

Distribution (Fig. 127). Coasts of Adriatic, Ionian and Aegean Seas: recorded from Croatia (new record), Greece and Italy.

Remarks. The relatively large bursa as depicted in the original description was not observed by me.

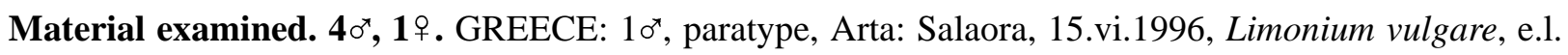
vi.1996, A. Laštůvka (RMNH); 10^, Fthiotis: Skala, 11.vi.1997, Limonium vulgare, e.1. vi.1997, Laštůvka (ZMUC). - ITALY: 20`, 1ㅇ, paratype, Brussa: Portogruaro, 15.viii.1997, Limonium vulgare, e.1. [viii].1997, A. Laštůvka (AL, CVDB, ZMUC).

Leafmines on Limonium cf. vulgare.: GREECE: Arta: Salaora, 15.vi.1996, A. Laštůvka (RMNH) [mixed with inseparable mines of maritima]. - ITALY: Brussa: Portogruaro, 16.viii.1997, A. Laštůvka (RMNH).

Additional records. CROATIA: $10^{\star}$, Dalmacia, Blace, Limonium vulgare, e.p. viii. 2002, A. Laštůvka (AL). 
Type material. Holotype ơ: GREECE (Lesvos): Lesvos: Skala Kallonis, 39.12'22”N-26.13'3”E, 16.vi.2004, M. Hull, leafmines on Limonium gmelini (Willd.), e.l. 20.vi-1.vii.2004, reared in Leiden, RMNH/EvN no. 2004902, Don. M. Hull 2004-ENT-56, Genitalia slide EvN 3625 (RMNH-INS no 23625). - Paratypes 16 ${ }^{x}$, 17우, 9 larvae, same locality, 13.vi.2003, 12, 13, $16+19 . v i .2004$, e.l. resp. 19-24.vi.2003, 20.vi6.vii.2004, M. Hull, reared in Caernarfon or Leiden; Genitalia slides ơ 3552, 우 3626, 우 3642, Wing slide 3645, Larval slides RMNH INS no 12392-12394 (RMNH, BMNH, WML, MH).

Non type material. Leafmines: same data as types.

Diagnosis. Externally A. lesbia resembles A. limonii, but the female lacks the conspicuous anal tuft. Males identified with certainty by examination of genitalia: gnathos triangular, wider than in limonii, but distinctly narrower than in maritima, uncus narrow and pointed as in limonii. Aedeagus distinctly longer than in maritima. Female genitalia resemble limonii in the hairy anal papillae and S7, but lack the conspicuous tufts on T7.

Description. Male (Figs. 14, 16, 17). Forewing length 1.7-2.1 mm, wingspan 4.0-4.6 mm. Head: frontal tuft and collar white or ochreous, mixed with dark grey scales; scape hidden in tuft, some brown-tipped scales, flagellum grey. Antenna with 28-34 segments. Thorax and forewing white, irrorate with brown-tipped scales; white fascia at $2 / 3$, some additional white spots basally may be present; cilia white, cilia line present. Hindwing narrow, grey, with costal bristles. Underside of forewing and hindwing dark brown or grey. Abdomen dark grey with conspicuous yellow anal tufts.

Female. Forewing length 1.9-2.2 mm, wingspan 4.4-4.8 mm. Antenna with 29-31 segments. Abdominal tip broadly rounded, no tufts present.

Male genitalia (Figs. 38, 44, 70, 82, 83). Measurements: see Table 3. Vinculum rounded, ventral plate narrow, with ventral, triangular posterior process; lateral arms slightly expanded ventrally. Tegumen forming distinctly produced, rounded pseuduncus with many setae dorsally. Uncus with long, pointed central element, strongly curved posteriorly. Gnathos with triangular central element, longer than broad, lateral arms not 'swollen'. Valva (Fig. 70) basally broadest; tip slightly produced inwards, with many thick setae dorsally; transtilla with slightly curved transverse bar and short sublateral processes. Aedeagus (Figs. 44, 82, 83) with ventral carina bifurcate, long, pointed; lateral carinae strongly curved dorsad. Vesica without cornuti.

Female genitalia (Figs. 57-59). Two groups of 6-8 setae along posterior margin of T7. T8 trapezoid, slightly indented mesially, without setae and scales. T9 with two groups of more than 50 setae. S7 also with many setae near abdominal tip. Vestibulum and bursa flimsy, without sclerotisations, bursa hardly reaching beyond segment 7, total bursa length from cloaca ca $375 \mu \mathrm{m}$. Ductus spermathecae with 2 narrow convolutions.

Final instar larva. Head capsule ca $350 \mu \mathrm{m}$ wide, see further group description.

Biology. Hostplant: Limonium gmelinii (det. J. Edmondson). The hostplant grows here on the edges of the dunes, either close to the sea in sand or close to the salt marsh amongst grass. The host does not grow on the salt marsh proper.

Egg on either side of leaf. Leafmine (Figs. 115, 116) a full-depth mine, gallery throughout, starting much contorted, often spirally, later a full-depth mine with narrow broken, brown, frass, following straighter course through leaf. Larval exit hole on leaf upperside. Mines not separable from those of A. maritima or A. limonii.

Cocoon white, usually spun on leaf underside (Fig. 117).

Distribution (Fig. 127). Greece, Lesvos. Only found at Skala Kallonis, and not found on other sites with the host on Lesvos, nor on Khios (pers. comm. M. Hull).

Etymology. Named after the island of Lesvos (=Lesbos): lesbios (Greek), lesbius (Latin), an adjective, denoting 'from Lesvos' or inhabitant of Lesvos. Lesvos is the transcription of modern Greek as used in the Times Atlas (Times 2000). 
Acalyptris limoniastri sp. $\mathbf{n}$.

(Figs. 10, 33-35, 51, 52, 60, 66, 76-78, 99, 126)

Trifurcula sp.; Walsingham 1904: 216.

Acalyptris sp. n.; van Nieukerken 1986b: 141 [listed]

Type material. Holotype ơ: ALGERIA: Biskra, 24.ii.1903, Wlsm. 96906; Walsingham Collection, B.M. 1910-427; Genitalia slide 22597 (BMNH). - Paratypes 26 $0^{x}$, 2우. ALGERIA: 17 $0^{x}, 2$ 우 same data as holotype, genitalia slides: BM22613-22615 (BMNH, 4 in RMNH); 10: Biskra, 19.iii.1894, Eaton (BMNH); $20^{\star}$ : Biskra, 1.v.1894, Eaton (BMNH); $50^{\star}$ : Hammam-es-Salahin, 1.iii. (30 ), 26.iii., 13.iv.1904, Walsingham, genitalia slide BM22616 (BMNH). TUNISIA: 10: Nefta, 14-16.iii.1986, Zool. Mus. Copenhagen Exp., genitalia slide EvN 3127 (ZMUC).

Diagnosis. A. limoniastri is recognised by a uniform white collar, irrorate with light brown, and absence of special scales, which separates it from Trifurcula species; other Acalyptris species are darker and/or have a distinct colour pattern. A. limoniastri is distinctly larger than the other species of its group. The male genitalia resemble those of the Limonium-feeders, but the species may be recognised by the more parallel-sided valva, the pointed pseuduncus (not to be confused with the uncus!) and band-shaped uncus (pointed in the other species); the female terminalia resemble those of A. limonii and A. lesbia, but A. limoniastri has more setae on T9 (more than 150), and the setae on $\mathrm{T} 7$ are absent.

Description. Male (Fig. 10). Forewing length 2.5-3.0 mm, wingspan 5.5-6.6 mm. Head: frontal tuft and collar white, occasionally with few ochreous scales; scape white with few ochreous scales, flagellum ochreous. Antenna with 43-46 segments. Thorax and forewing white, irrorate with light brown-tipped scales; cilia white, no distinct cilia line. Hindwing white, with costal bristles. Underside of forewing ochreous, hindwing white. Abdomen yellowish white, with long ochreous anal tufts.

Female. Forewing length $2.96 \mathrm{~mm}$, wingspan $6.4 \mathrm{~mm}$. Antenna with 38 segments.

Male genitalia (Figs. 33-35, 66, 76-78). Measurements: see Table 3. Vinculum anteriorly narrowed, truncate; lateral arms expanded ventrally. Tegumen pointed, curved ventrad. Uncus ribbon-like, without central element, with prominent seta (or two) on both sides laterally. Gnathos with long narrow central element. Valva approximately parallel sided, inner margin serrate; tip not demarcated, with many spine-like setae; transtilla with distinct straight transverse bar and long sublateral processes. Aedeagus widening posteriorly, with ventral bilobed carina, tightly fused to ventral process of vinculum; and pair of lateral carinae, tips pointed, curved dorsad. Vesica without cornuti.

Female genitalia (Figs. 51, 52). T8 narrow, band-like, with 15-17 long setae and scales on each half. T9 cushion-shaped, with large group of more than 150 setae in total. S7 medially with dense group of spine-like setae near tip. Anterior apophyses reaching beyond posterior apophyses. Vestibulum not very distinct, without sclerotisations. Bursa flimsy, hardly visible, without sclerotisations, hardly reaching beyond segment 7 . Ductus spermathecae with $2-2 \frac{1}{2}$ narrow convolutions.

Biology. Immature stages unknown. The species was collected by Walsingham (1904) from bushes of Limoniastrum guyonianum on which he later found some mines, very likely to belong to this species. A few mined leaves on a pin were placed in the same drawer as the type series. The mine can be described as follows: a narrow gallery following leaf margin upwards and down again along other side; frass in narrow midline. Limoniastrum guyonianum is a common bush in open desert shrub near Biskra. The author searched in vain for mines or adults of this species on the hostplant near Biskra in May 1980.

Distribution (Fig. 126). Northern fringes of Sahara in Algeria and Tunisia.

Etymology. A noun in genitive case, named after the supposed host genus Limoniastrum. 
Acalyptris pyrenaica A. \& Z. Laštůvka, 1993: 158. Holotype ơ SPAIN: Cataluna, Lerida, Coll del Canto, (E Sort), 1650 m, 21.vi.1992, A. Laštůvka (AL) [examined]

Acalyptris pyrenaica; van Nieukerken 1996: 24 [catalogued]; van Nieukerken \& Biesenbaum 1997: 2 [redescription]; A. \& Z. Laštůvka 1997: 120 [description]; van Nieukerken et al. 2004a: 227 [Iberian Peninsula].

Material examined. 4 $0^{x}, 1$ 우. GERMANY: $10^{x}$, Nordrhein-Westfalen: Nordeifel, Bad Münstereifel, NSG

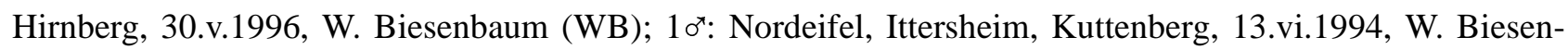
baum (WB); 1 o $^{x}$ Nordeifel, Marmagen, Gillesbachtal, 29.vi.1995, W. Biesenbaum (WB); $10^{x}$ : Nordeifel, Nettersheim, Urftal, 17.vi.1994, W. Biesenbaum (RMNH). SPAIN: 1ㅇ, Cataluna, Lerida, Coll del Canto, (E Sort), 1650 m, 21.vi.1992, A. Laštůvka (AL).

Diagnosis. A. pyrenaica is characterised by the complete black colour, which separates it from most other European Nepticulidae; it only slightly resembles some Parafomoria species, but these usually with at least some white scaling. The male genitalia resemble other species in this group, but A. pyrenaica has a double uncus and simpler, narrow valva.

Description Male (Fig. 15). Forewing length 2.0-2.5 mm, wingspan 4.0-5.6 mm. Head: frontal tuft and collar dark grey to anthracite black; scape black with some white scales along frontal edge, forming very small eyecap; flagellum grey, antenna with 36-39 segments. Thorax and forewing dark grey to anthracite black, at forewing tip paler bases of scales are more exposed, yielding speckled appearance. Underside dark grey. Hindwing completely grey on both surfaces, costal bristles present. Abdomen completely black, conspicuous long anal tufts mixed pale grey with black.

Female (from original description). Wingspan $5.1 \mathrm{~mm}$, antenna with 33 segments. Further as male.

Male genitalia (Figs. 41, 71). Measurements: see Table 3. Vinculum with narrow ventral plate. Tegumen forming blunt pseuduncus. Uncus with several setae laterally and complex sclerotized tip: a posterio-dorsal bilobed element and more ventrad blunt element. Gnathos with narrow, relatively long central element. Valva slender, with almost parallel margins, with slight inner bulge at basis; near tip with many setae. Transtilla with distinct transverse bar and relatively short sublateral processes. Aedeagus distally widened, with pair of dorsolateral carinae, each split in 2-4 points; ventral carina with short distal points, wide apart. Vesica without any cornuti.

Female genitalia (Fig. 50). Probably incomplete in slide. Short anal papillae. Apophyses anteriores and in particular posteriores long. Ductus spermathecae with 2 convolutions. Corpus bursae not seen.

Biology. Unknown, all adults were taken by sweeping grassland vegetation in May to July. The habitats were limestone grassland in the Eifel mountains and a mountain pasture in the Pyrenees. The hostplant cannot belong to the Plumbaginaceae; although a species of Armeria is present in the type-locality, it does not occur in the German localities.

Distribution (Fig. 126). Only known from Spain, Pyrenees and Germany, Eifel.

\section{Discussion}

\section{Barcodes}

In Fig. 120 the neighbour joining tree shows the relative distances (K2P distances) between the analysed CO1 sequences. The two sequences of A. loranthella, belonging to the same population, are identical, and two of the three sequences of $A$. lesbia, also from one population, are identical, a third differs in just one synonymous substitution (sequence divergence $=0.15 \%$ - 1 nucleotide). The three sequences of the geographical distant specimens of A. platani (France, Greece and Turkey), differ 1.8-2.3\% (11 to 15 nucleotides). Differences 
between the species were always higher, the smallest difference found is that between the species $A$. limonii and A. lesbia: 3.2-3.4\%. In the pairs A. minimella and A. pistaciae and A. maritima and A. limonii the differences are in both cases $7.1 \%$. Although it represents a small sampling of the taxon, $\mathrm{CO} 1$ is able to identify the species reliably. This can be especially useful for the separation of the morphologically inseparable mines and larvae on Limonium in the East Mediterranean and for the otherwise inseparable mines of A. minimella and A. pistaciae on Pistacia. This may be important in the area between the currently known distributions, where the species might be sympatric.

\section{Phylogeny}

The maximum parsimony analysis (exhaustive search) of the CO1 sequences found three shortest trees, with a length of 319 steps, consistency index $=0.683$ and retention index $=0.685$. Of 665 characters, 104 are parsimony informative. The strict consensus tree (Fig. 121) is fully resolved to the species level, the only difference between the three trees are the relative positions of the individual sequences of $A$. platani. The monophyly of Acalyptris and the platani and staticis species groups is recovered and the pairing of A. limonii and lesbia is also supported.

For a full phylogeny of Acalyptris, many more species from different species groups and continents should be included, nuclear markers should be added and also a morphological analysis should be carried out. The genus Acalyptris is supported by one distinct morphological apomorphy: venation with the closed cell in forewing shifted towards base, almost vestigial. In addition there is another possible generic apomorphy in the larva: anal rod with three processes. This character ought to be checked in larvae of other groups; in the only other larva described (the North-American A. lotella (Wagner, 1987)), this character was not examined (Wagner 1987).

Both species groups have some remarkable morphological features within Nepticulidae. The platani group are the only checked Trifurculini with a subdorsal retinaculum, similar to the Nepticulini and also noted in the Australian species (Hoare 1998).

The staticis group particularly has a number of unusual characters: the female genitalia have only a reduced and very small bursa, whereas the male genitalia have a poorly-developed vesica, without cathrema or any sclerotisations: these characters are almost certainly correlated and related to the way mating is performed.

The larva of A. lesbia (the only studied species in the group) is also remarkable by the very low number of setae on meso- and metathorax in the final instar and the overall small size of most setae relative to other Nepticulidae; furthermore it differs in the length of the tentorial arms and the shape of the frontoclypeus: characters otherwise considered sufficient to separate genera.

The pupae of the two groups also differ markedly: the frons in the platani group has a conical projection similar to that in Trifurcula (see van Nieukerken et al. 2004b) and the frons is detaching completely during ecdysis, a character unknown in other Nepticulidae, but also noted in an Australian Acalyptris feeding on Loranthaceae (Hoare 1998); the pupa in the staticis group does not show these characters.

It is clear that the species groups discussed here are far apart taxonomically. The present data, however, do not allow to reject the monophyly of Acalyptris: the other sizeable groups, such as the repeteki group (with the type species) and the New World groups (formerly placed in Microcalyptris), should be included in a future phylogenetic analysis, preferably including the hardly known immatures.

\section{Hostplant relationships}

The species of the platani group feed on a wide array of angiosperm families, both on woody plants and on 
plant parasites (Loranthaceae). There is no compelling phylogenetic pattern to be distinguished, many hosts are Rosids, but also basal Eudicots and Asterids are represented. The few species and host associations in Europe probably represent a relict from a larger tropical fauna during the Tertiary. Two of the three European host families, Loranthaceae and Anacardiaceae, are also represented as hosts in other parts of the world, as distant as Australia (Loranthaceae) and South America (Anacardiaceae), but Anacardiaceae are also used by a species in China.

The staticis group, on the other hand, is almost completely specialised on Plumbaginaceae, belonging to the basal Eudicot order Caryophyllales (Bremer et al. 2003), and four out of six species are specialised on Limonium species growing in coastal communities. Only one species, A. limoniastri, is known from inland desert habitats, but it is not unlikely that more Acalyptris species of this group will found in similar inland habitats. The local distribution of the hosts on isolated localities has probably played a role in the speciation of these species. It is interesting to note that two other old world groups in Acalyptris confined to desert regions — the repeteki and shafirkanus groups - have unknown biologies. Furthermore the plant order Caryophyllales comprises several other families that are specialised and often dominant desert plants; examples are the Tamaricaceae, Frankeniaceae and Amaranthaceae s.l. In appropriate sites, searching these plants for the unknown Acalyptris larvae may be the first option to try.

\section{Acknowledgements}

Many people assisted and supported me in the course of the more than 20 years that I gathered data for this study. I am grateful for the loan or gift of material and for information to: Giorgio Baldizzone (Asti, Italy), Graziano Bassi (Torini, Italy), Kees van den Berg (Leiden, the Netherlands), Willy Biesenbaum (Velbert-Langenberg, Germany), Jaroslaw Buszko (Torún, Poland), the late Roger Buvat (Marseille, France), Martin F.V. Corley (Faringdon, United Kingdom), J. Hans Donner (Amsterdam, the Netherlands), Bert Gustafsson (Stockholm, Sweden), Robert Hoare (Auckland, New Zealand), Michael Hull (Gwynned, United Kingdom), Roland Johansson (Växjö, Sweden), Lauri Kaila (Helsinki, Finland), the late Joseph Klimesch (Linz, Austria), the late Friedrich Kasy (Vienna, Austria), Aleš Laštůvka (Prostejov, Czech Republic), Zdeněk Laštůvka (Brno, Czech Republic), Ole Karsholt (Copenhagen, Denmark), Martin Lödl (Vienna, Austria), Wolfram Mey (Berlin, Germany), Reinhard Sutter (Bitterfeld, Germany), Zdenko Tokár (Michalovce, Slovakia), the late Ernst Traugott-Olsen (Marbella, Spain), Paolo Triberti (Verona, Italy), and Kevin Tuck (London, United Kingdom).

A special word of thanks is due to Michael Hull for allowing me to include the joint description of the species collected and reared by him on Limonium in Lesvos. Kees van Achterberg (Leiden, the Netherlands) provided information on Braconidae and John Edmondson (Liverpool Museum) provided information on the taxonomy of Limonium to Michael Hull. I thank my colleagues Kees van den Berg, who skilfully made several preparations of larvae and genitalia and assisted with rearing of material and Dick Groenenberg for all the labwork to obtain the barcode sequences, for advise on molecular methods and comments on the manuscript.

This study started during my doctoral candidacy at the Department of animal systematics and biogeography (Vrije Universiteit, Amsterdam), and I am happy to be able to include some results of former students Ruud Ph. Jansen (Amsterdam, the Netherlands), Remko W. G. Andeweg (Rotterdam, the Netherlands) and Henk Dop (Amsterdam, the Netherlands).

Robert Hoare (Auckland, New Zealand), Jan van Tol (Leiden, the Netherlands), Don Davis (Washington DC, USA) and David Wagner (Storrs, CO, USA) are acknowledged for their critical and helpful reviews of this manuscript. 


\section{References}

Achterberg, C. van (1983) Revisionary notes on the subfamily Gnaptodontinae with description of eleven new species (Hymenoptera, Braconidae). Tijdschrift voor Entomologie, 126 (1-2), 25-57.

Amsel, H.G. \& Hering, M. (1931) Beitrag zur Kenntnis der Minenfauna Palästinas. Deutsche Entomologische Zeitschrift, 1931, 113-152, pls 1-2.

Arenberger, E. \& Wimmer, J. (1996) Erster Nachtrag zur Mikrolepidopterenfauna Zyperns. Nachrichten des Entomologischen Vereins Apollo, N. F., 17 (2), 209-224.

Askew, R.R. (1984) Variation in Cirrospilus vittatus Walker (Hym; Eulophidae) and the description of a new species from Britain. Entomologist's Monthly Magazine, 120, 63-68.

Bremer, B., Bremer, K., Chase, M.W., Reveal, J.L., Soltis, D.E., Soltis, P.S., Stevens, P.F., Anderberg, A.A., Fay, M.F., Goldblatt, P., Judd, W.S., Kallersjo, M., Karehed, J., Kron, K.A., Lundberg, J., Nickrent, D.L., Olmstead, R.G., Oxelman, B., Pires, J.C., Rodman, J.E., Rudall, P.J., Savolainen, V., Sytsma, K.J., van der Bank, M., Wurdack, K., Xiang, J.Q.Y. \& Zmarzty, S. (2003) An update of the Angiosperm Phylogeny Group classification for the orders and families of flowering plants: APG II. Botanical Journal of the Linnean Society, 141 (4), 399-436.

Brown, P.A. (1997) A review of techniques used in the preparation, curation and conservation of microscope slides at the Natural History Museum, London. Biology Curator, 10 (special supplement), 1-33.

Buhr, H. (1940) Über Verbreitung und ausländische Nahrungspflanzen von Neptikeln. Zeitschrift des Wiener Entomologen-Vereins, 25, 193-196, 209-211, 226-235.

Corley, M.F.V., Gardiner, A.J., Cleere, N. \& Wallis, P.D. (2000) Further additions to the Lepidoptera of Algarve, Portugal (Insecta: Lepidoptera). SHILAP Revista de Lepidopterologia, 28 (111), 245-319.

Davatchi, G.A. (1958) Étude biologique de la Faune entomologique des Pistacia sauvages et cultivés. Revue de Pathologie Végétale et d'Entomologie Agricole de France, 37 (1), 3-166.

Diškus, A. \& Puplesis, R. (2003) Catalogue of the world Nepticuloidea \& Tischerioidea. In: Puplesis, R. \& Diškus, A. (Eds) Nepticuloidea ir Tischerioidea (Lepidoptera) pasaulio ir lietuvos faunoje. The Nepticuloidea \& Tischerioidea (Lepidoptera) - a global review, with strategic regional revisions, Lutute publishers, Kaunas, pp. 318-436.

Drăghia, I. (1968) Données concernant la biologie de quelques microlépidoptères mineurs. Travaux du Muséum d'Histoire Naturelle "Grigore Antipa", 8, 857-863, 6 pls.

Erdös, J. (1956) Additamenta ad cognitionem faunae Chalcidoidarum in Hungaria et regionibus finitmis. VI. 19. Eulophidae. Folia Entomologica Hungarica, s.n., 9 (1), 1-64.

Evenhuis, N.L. \& Samuelson, G.A. (2004) The insect and spider collections of the world website. Bishop Museum. Available from http://hbs.bishopmuseum.org/codens/codens-r-us.html. (Accessed 21 June 2006.)

Groschke, F. (1944) Neues über Minierer aus dem Mittelmeergebiet. Mitteilungen der Münchner Entomologischen Gesellschaft, 34, 115-124.

Gustafsson, B. (1981) New leaf-mining moths of the family Nepticulidae from Cyprus, Greece (Lepidoptera). Entomologica Scandinavica, 12 (4), 453-469.

Gustafsson, B. \& Nieukerken, E.J. van (1990) Larvae of Nepticulidae. In: Johansson, R., Nielsen, E.S., Nieukerken, E.J. van \& Gustafsson, B. (Eds) The Nepticulidae and Opostegidae (Lepidoptera) of NW Europe, Fauna Entomologica Scandinavica, 23, E. J. Brill, Leiden, pp. 323-356.

Hall, T.A. (2004) BioEdit sequence alignment editor, Version 7.0.1. Isis Pharmaceuticals.

Hansson, C. (1985) Taxonomy and biology of the Palearctic species of Chrysocharis Förster, 1856 (Hymenoptera: Eulophidae). Entomologica Scandinavica. Supplementum, 26, 1-130.

Hansson, C. (1990) A taxonomic study on the Palaearctic species of Chrysonotomyia Ashmead and Neochrysocharis Kurdjumov (Hymenoptera: Eulophidae). Entomologica Scandinavica, 21 (1), 29-52.

Hebert, P.D.N., Penton, E.H., Burns, J.M., Janzen, D.H. \& Hallwachs, W. (2004) Ten species in one: DNA barcoding reveals cryptic species in the neotropical skipper butterfly Astraptes fulgerator. Proceedings of the National Academy of Sciences of the United States of America, 101 (41), 14812-14817.

Hering, M. (1957) Bestimmungstabellen der Blattminnen von Europa, 3 vols, Junk, s-Gravenhage, 1185 pp.

Hoare, R.J.B. (1998) Systematics of Australian Nepticulid moths (Lepidoptera: Nepticulidae), Thesis from Australian National University, Canberra, 248 pp.

Kasy, F. (1969) Lepidopterologisch-faunistisch bemerkenswerte Neufunde aus Niederösterreich, V. Zeitschrift der Arbeitsgemeinschaft Österreichischer Entomologen, 21 (1), 2-4.

Klimesch, J. (1937) Nepticula loranthella spec. nov. (Lep. Nepticulidae). Zeitschrift des Österreichischen EntomologenVereins, 22, 33-35.

Klimesch, J. (1940) Über eine Nepticuliden-Ausbeute von Triest sowie Beschreibung der Mine von Leucospilapteryx cupediella H.S. (Lep. Nepticulidae et Gracilariidae). Zeitschrift des Wiener Entomologen-Vereines, 25, 176-179, 188-193, pl. 21.

Klimesch, J. (1942) Über Microlepidopteren-Ausbeuten aus der gegend von Zaton bei Gravosa (Süddalmatien). Mittei- 
lungen der Münchner Entomologischen Gesellschaft, 32, 347-399, pls 13-15.

Klimesch, J. (1948) Zur Frage der verwantschaftlichen Beziehungen einiger Stigmella-Arten auf Grund des Baues des männl. Kopulationsapparates. (Lep., Stigmellidae). Zeitschrift der Wiener Entomologischen Gesellschaft, 33, 49-82.

Klimesch, J. (1951) Contributo alla fauna Lepidotterologica del Trentino. Studi Trentini di Scienze Naturali, 27 (1-3), $11-68$.

Klimesch, J. (1953) Die europäischen Trifurcula- und Ectoedemia-Arten (Lep., Nepticulidae). Zeitschrift der Wiener Entomologischen Gesellschaft, 38, 160-170, 191-196.

Klimesch, J. (1968) Die Lepidopterenfauna Mazedoniens, 4. Microlepidoptera. Posebno Izdanie. Prirodonaucen Muzej Skopje, 5, 1-201.

Klimesch, J. (1977) Beiträge zur Kenntnis der Microlepidopteren-Fauna des Kanarischen Archipels. 1. Beitrag: Nepticulidae, Tischeriidae. Vieraea, 6, 191-218.

Klimesch, J. (1978) Beitrag zur Kenntnis der Nepticulidenfauna von Anatolien und der Insel Rhodos (Lepidoptera, Nepticulidae). Tijdschrift voor Entomologie, 121 (5), 239-278.

Knölke, S., Erlacher, S., Hausmann, A., Miller, M.A. \& Segerer, A.H. (2005) A procedure for combined genitalia dissection and DNA extraction in Lepidoptera. Insect Systematics and Evolution, 35 (4), 401-409.

Kuchlein, J.H. (2002) Caryocolum proxima Haworth (Lepidoptera: Gracillariidae) [sic!] en Acalyptris platani MüllerRutz (Lepidoptera: Nepticulidae) nieuw voor de Nederlandse fauna. Tinea Nederland, 1 (1), 7.

Kuchlein, J.H., Holt, J. ten \& Kuchlein-Nijsten, C.M. (2003) Préludes. Tinea Nederland, 1 (2), 16.

Laštůvka, A. \& Laštůvka, Z. (1990) Zur Faunistik der Nepticulidae-Arten in der Tschechoslowakei (Lepidoptera). Casopis Moravskeho Muzea Vedy Prirodni, 75 (1-2), 185-192.

Laštůvka, A. \& Laštůvka, Z. (1993) Acalyptris pyrenaica sp. n. aus Spanien (Lepidoptera: Nepticulidae). SHILAP Revista de Lepidopterologia, 21 (83), 157-160.

Laštůvka, A. \& Laštůvka, Z. (1997) Nepticulidae Mitteleuropas. Ein illustrierter Begleiter (Lepidoptera), Konvoj, Brno, $229 \mathrm{pp}$.

Laštůvka, A. \& Laštůvka, Z. (2005) Four new Trifurcula species and additional faunal data on Nepticulidae from Italy (Lepidoptera: Nepticulidae). Acta Universitatis Agriculturae et Silviculturae Mendelianae Brunensis, 53 (1), 7-14.

Laštůvka, Z. \& Laštůvka, A. (1998) Beitrag zur Kenntnis der Nepticulidenfauna Griechenlands (Lepidoptera, Nepticulidae). Stapfia, 55, 313-326.

Lhomme, L. (1934) Excursion au pays des mines et description d'une sous-espèce nouvelle de Lithocolletis. Amateur de Papillons, 7, 108-112, 113-121, 129-138, 161-169.

Lo Duca, R., Massa, B. \& Concetta Rizzo, M. (2002) Importanza deo fragmenti di habitat naturale per le comunità di fillominatori (Insecta Diptera, Lepidoptera et Hymenoptera) e loro parassitoidi (Hymenoptera Eulophidae). Atti dell'Accademia Roveretana degli Agiati B, Classe di Scienze matematiche, fisiche e naturali, (8) 2, 51-122.

Massa, B., Concetta Rizzo, M. \& Caleca, V. (2001) Natural alternative hosts of Eulophidae (Hymenoptera: Chalcidoidea) parasitoids of the Citrus leafminer Phyllocnictis citrella Stainton (Lepidoptera: Gracillariidae) in the Mediterranean Basin. Journal of Hymenoptera Research, 10 (1), 91-100.

Mehrnejad, M.R. (2001) The current status of pistachio pests in Iran. Cahiers Options Mediterraneennes, 56, 315-322.

Morton, A. (2000) DMAP for Windows, Version 7.0e. Alan Morton, Winkfield, Windsor, Berkshire. Available from http:/ /www.dmap.co.uk/welcome.htm

Müller-Rutz, J. (1934a) Über Microlepidopteren. Mitteilungen der Schweizerischen Entomologischen Gesellschaft, 16 (2), 118-128, 1 pl.

Müller-Rutz, J. (1934b) Berichtigung. Mitteilungen der Schweizerischen Entomologischen Gesellschaft, 16 (3), slip.

Nieukerken, E.J. van (1982) Report of a collecting trip to Greece by S.B.J. Menken and E.J. van Nieukerken, 1980, primarily to collect Nepticulidae (Lepidoptera). Amsterdam, Vakgroep Biosystematiek Biologisch Laboratorium Vrije Universiteit, 1-23.

Nieukerken, E.J. van (1983) The Cistaceae-feeding Nepticulidae (Lepidoptera) of the western Palaearctic region. Systematic Entomology, 8 (4), 453-478.

Nieukerken, E.J. van (1985) A taxonomic revision of the western Palaearctic species of the subgenera Zimmermannia Hering and Ectoedemia Busck s. str. (Lepidoptera, Nepticulidae), with notes on their phylogeny. Tijdschrift voor Entomologie, 128 (1), 1-164.

Nieukerken, E.J. van (1986a) Systematics and phylogeny of Holarctic genera of Nepticulidae (Lepidoptera, Heteroneura: Monotrysia). Zoologische Verhandelingen, 236, 1-93.

Nieukerken, E.J. van (1986b) A provisional phylogenetic check-list of the western Palaearctic Nepticulidae, with data on hostplants (Lepidoptera). Entomologica Scandinavica, 17 (1), 1-27.

Nieukerken, E.J. van \& Dop, H. (1987) Antennal sensory structures in Nepticulidae (Lepidoptera) and their phylogenetic implications. Zeitschrift für Zoologische Systematik und Evolutionsforschung, 25 (2), 104-187.

Nieukerken, E.J. van \& Johansson, R. (1990) Tribus Trifurculini. In: Johansson, R., Nielsen, E.S., Nieukerken, E.J. van \& Gustafsson, B. (Eds) The Nepticulidae and Opostegidae (Lepidoptera) of NW Europe, Fauna Entomologica Scandinavica, 23, pp. 239-321. 
Nieukerken, E.J. van, Nielsen, E.S., Johansson, R. \& Gustafsson, B. (1990) Introduction to the Nepticulidae. In: Johansson, R., Nielsen, E.S., Nieukerken, E.J. van \& Gustafsson, B. (Eds) The Nepticulidae and Opostegidae (Lepidoptera) of NW Europe, Fauna Entomologica Scandinavica, 23, pp. 11-109.

Nieukerken, E.J. van (1996) Nepticulidae, Opostegidae. In: Karsholt, O. \& Razowski, J. (Eds) The Lepidoptera of Europe. A distributional checklist, Apollo Books, Stenstrup, pp. 21-27, 300.

Nieukerken, E.J. van \& Biesenbaum, W. (1997) Nachweis von Acalyptris pyrenaica Laštůvka \& Laštůvka, 1993 in der Nordeifel - ein Neufund für Deutschland (Lep., Nepticulidae). Melanargia, 9 (1), 1-9.

Nieukerken, E.J. van \& Laštůvka, A. (2002) Ectoedemia (Etainia) obtusa Puplesis \& Diškus, new for Europe: taxonomy, distribution and biology (Lepidoptera: Nepticulidae). Nota Lepidopterologica, 25 (2/3), 87-95.

Nieukerken, E.J. van (2004) Fauna Europaea: Crinopterygidae, Heliozelidae, Nepticulidae, Opostegidae, Schreckensteiniidae, Urodidae. - In: Karsholt, O. \& E. J. van Nieukerken (eds.) 2004. Lepidoptera, Moths. Fauna Europaea version 1.1. Fauna Europaea, Copenhagen, Amsterdam, Paris. Available from http://www.faunaeur.org/. (accessed 21 June 2006)

Nieukerken, E.J. van, Laštůvka, A. \& Laštůvka, Z. (2004a) Annotated catalogue of the Nepticulidae and Opostegidae (Lepidoptera: Nepticuloidea) of the Iberian Peninsula. SHILAP Revista de Lepidopterologia, 32 (127), $211-260$.

Nieukerken, E.J. van, Mazurkiewicz, A. \& Pałka, K. (2004b) Trifurcula pallidella (Duponchel, 1843) (Nepticulidae): distribution, biology and immature stages, particularly in Poland. Nota Lepidopterologica, 27 (2/3), 159-178.

Nieukerken, E.J. van, Laštůvka, A. \& Laštůvka, Z. (2006) Annotated catalogue of the Nepticulidae and Opostegidae (Lepidoptera: Nepticuloidea) of France and Corsica. Zootaxa, 1216, 1-114.

NIMA (2004) GEOnet Names Server. National Imagery and Mapping Agency. Available from http://gnswww.nima.mil/ geonames/GNS/index.jsp. (Accessed 21 June 2006.)

Noyes, J.S. (2003) Universal Chalcidoidea Database. Available from www.nhm.ac.uk/entomology/chalcidoids/ index.html. (Accessed 23 March 2006)

Olivella, E. (2000) Aportació al coneixement dels lepidòpters minadors de Catalunya. Butlleti de la Societat Catalana de Lepidopterologia, 85, 27-36.

Papp, J. (1989) Mirax heinrichi sp. n. from the U.S.A. and taxonomic bionomic data of two European Mirax species (Hymenoptera: Braconidae, Adeliinae). Folia Entomologica Hungarica, 50, 105-110.

Passos de Carvalho, J. \& Corley, M.F.V. (1995) Additions to the Lepidoptera of Algarve, Portugal (Insecta: Lepidoptera). SHILAP Revista de Lepidopterologia, 23 (91), 191-230.

Popescu-Gorj, A., Olaru, V. \& Drăghia, I. (1972) Ord. Lepidoptera. L'entomofaune du "Grind" de Caraorman, delta du Danube. Travaux du Muséum d'Histoire Naturelle "Grigore Antipa", 12, 181-206.

Puplesis, R. (1990) The genus Acalyptris Meyrick (Lepidoptera, Nepticulidae) in the USSR: distribution and taxonomy. Nota Lepidopterologica, 13 (1), 62-88.

Puplesis, R. (1994) The Nepticulidae of eastern Europe and Asia. Western, central and eastern parts, Backhuys Publishers, Leiden, $290 \mathrm{pp}$.

Puplesis, R. \& Diškus, A. (1995) Acalyptris argyraspis sp. n., a remarkable species from Tadzhikistan (Lepidoptera: Nepticulidae). Phegea, 23 (1), 51-54.

Puplesis, R. \& Robinson, G.S. (2000) A review of the Central and South American Nepticulidae (Lepidoptera) with special reference to Belize. Bulletin of the Natural History Museum London. Entomology, 69 (1), 3-114.

Puplesis, R., Diškus, A. \& Robinson, G.S. (2002a) New Neotropical Nepticulidae (Lepidoptera) from the western Amazonian rainforest and the Andes of Ecuador. Bulletin of the Natural History Museum London. Entomology, 71 (1), 19-58.

Puplesis, R., Diškus, A., Robinson, G.S. \& Onore, G. (2002b) A review and check list of the Neotropical Nepticulidae (Lepidoptera). Bulletin of the Natural History Museum London. Entomology, 71 (1), 59-76.

Puplesis, R. \& Diškus, A. (2003) Strateginiu regionu taksonomines revizijos ir nauju rusiu aprasai. Strategic regional revisions with description of new species. In: Puplesis, R. \& Diškus, A. (Eds) Nepticuloidea ir Tischerioidea (Lepidoptera) pasaulio ir lietuvos faunoje. The Nepticuloidea \& Tischerioidea (Lepidoptera) - a global review, with strategic regional revisions, Lutute publishers, Kaunas, pp. 176-289.

Rebel, H. (1926) Beitrag zur Microlepidopterenfauna Dalmatiens. Verhandlungen der Zoologisch-Botanischen Gesellschaft in Wien, 74, (106)-(110).

Rizzo, M.C. \& Massa, B. (2002) Ecology of the eulophid parasitoid community living on hosts of spontaneous flora linked to citrus grove (Hymenoptera: Chalcidoidea: Eulophidae). In: Melika, G. \& Thuróczy, C. (Eds) Parasitic wasps: evolution, systematics, biodiversity and biological control. International symposium: "Parasitic Hymenoptera: taxonomy and biological control"(14-17 May 2001, Köszeg, Hungary), Agroinform Kiadó \& Nyomda, Budapest, pp. 351-361.

Scoble, M.J. (1980) The genus Niepeltia Strand: taxonomy, and comments on structure and relationships (Lepidoptera: Nepticulidae). Annals of the Transvaal Museum, 32 (8), 197-229.

Sefrová, H. (2001) Phyllonorycter platani (Staudinger) - a review of its dispersal history in Europe (Lepidoptera, Gracillariidae). Acta Universitatis Agriculturae et Silviculturae Mendelianae Brunensis, 49 (5), 71-76. 
Skala, H. (1938) Einiges über Falter-Minen aus dem Mediterrangebiet. Zeitschrift des Österreichischen EntomologenVereins, 23, 8-10, pls 6-7, 30-31, 43-46.

Strand, E. (1934) Niepeltia novum genus Lepidopterorum. Internationale Entomologische Zeitschrift, 28, 241.

Swofford, D.L. (2001) Paup, version 4.0b10 for 32-bit Microsoft Windows. Sinauer Ass., Sunderland, Massachusetts.

Szőcs, J. (1978) Adatok a Pili-hegység aknázómoly faunájához. (Data to the mining moths fauna from the Pilis Mts.). Folia Entomologica Hungarica, s.n., 31 (2), 265-271.

Szöcs, J. (1979) Angaben zu den Parasiten der minirenden Motten (Hymenoptera Braconidae). Folia Entomologica Hungarica, s.n., 32 (2), 199-206.

Times (2000) The Times comprehensive atlas of the world, 10th edition 911, Times Books, London, 544 pp.

Tokár, Z., Richter, I., Pastorális, G. \& Slamka, F. (2002) New and interesting records of Lepidoptera of Slovakia from the years 1998-2001. Entomofauna Carpathica, 14, 1-11.

Tudor, C. \& Drãghia, I. (1978) Chalcidoidea (Hymenoptera) parasites des larves de Microlépidoptères mineurs. Travaux du Muséum d'Histoire Naturelle "Grigore Antipa", 19, 315-319.

Tutin, T.G. et al. (Eds), (1964-1993) Flora Europaea, 5 vols, University Press, Cambridge.

Utech, L. (1962) Blattminen und Pflanzengallen aus Albanien und dem Kaukasus. Deutsche Entomologische Zeitschrift, N.F., $9(3 / 4), 229-235$.

Wagner, D.L. (1987) A new Microcalyptris species from California (Lepidoptera: Nepticulidae). Pan-Pacific Entomologist, 63 (3), 278-283.

Walsingham, L. (1904) Algerian Microlepidoptera. Entomologist's Monthly Magazine, 40 (9), 214-223.

Walsingham, L. (1908) Microlepidoptera of Tenerife. Proceedings of the Zoological Society of London, 1907, 911-1034, pls. 51-53.

Wieser, C., Huemer, P. \& Stangelmaier, G. (2002) Schmetterlinge (Lepidoptera). [In B. Gutleb \& C. Wieser, Ergebnisse einer zoologischen Excursion in den Nordiran, 2001]. Carinthia II, 192/112, 52-81.

Wilkinson, C. (1979) A taxonomic study of the micro-lepidopteran genera Microcalyptris Braun and Fomoria Beirne occurring in the United States of America (Lepidoptera, Nepticulidae). Tijdschrift voor Entomologie, 122 (4), 5990. 

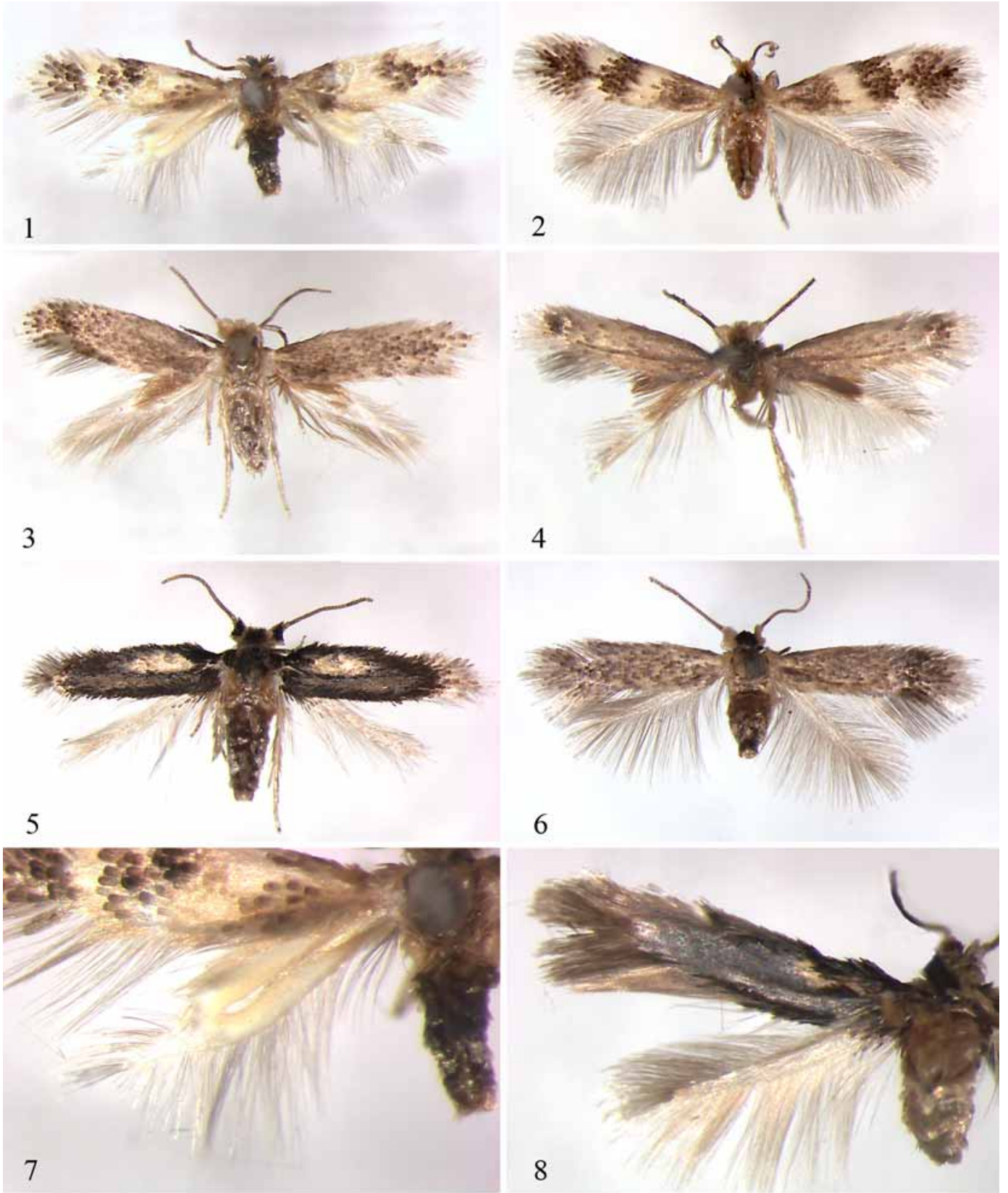

FIGURES 1-8. Acalyptris platani group, adults. 1, A. platani, ๙ ${ }^{\Uparrow}$, Turkey, Alanya; 2, A. platani, 우, Italy, Trieste; 3, A. minimella, $\sigma^{\star}$, Corsica, Porticcio; 4, A. pistaciae, $\sigma^{x}$, Greece, Dhelfoi, paratype; 5, A. loranthella, $\sigma^{\star}$, Czech Republic, Milovice; 6, A. loranthella, , , Greece, Evvoia, Dhírfis Oros; 7, A. platani, $\sigma^{\star}$, as 1, details of hindwing with special scales; 8, A. loranthella, ox, underside of wings, Czech Republic, Milovice; [material in RMNH, except Fig. 1, in ZMHB]. 

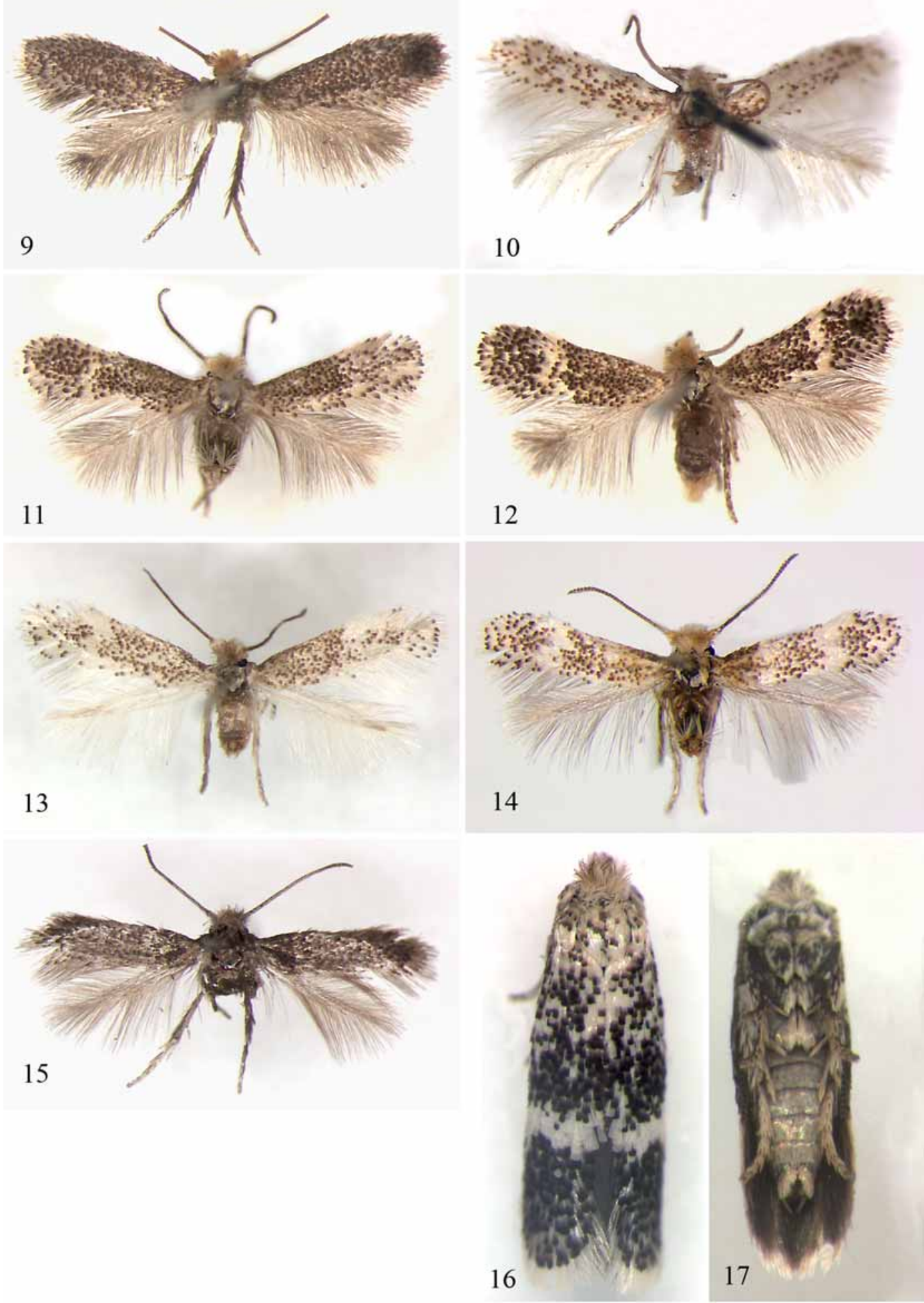

FIGURES 9-17. Acalyptris staticis group, adults. 9, A. staticis, Canary islands, Tenerife, Puerto de la Cruz; 10, A. limoniastri, Algeria, Biskra, Paratype; 11, A. limonii, ơ paratype, Greece, Salaora; 12, A. limonii, 우 paratype, Greece, Salaora; 13, A. maritima, ơ, Greece, Skala; 14, A. lesbia, ơ holotype, Greece, Lesvos; 15, A. pyrenaica, Germany, Eifel, Nettersheim; 16, 17, A. lesbia, $\sigma^{x}$ live specimen, paratype; [material in RMNH, except Fig. 13, in ZMUC]. 


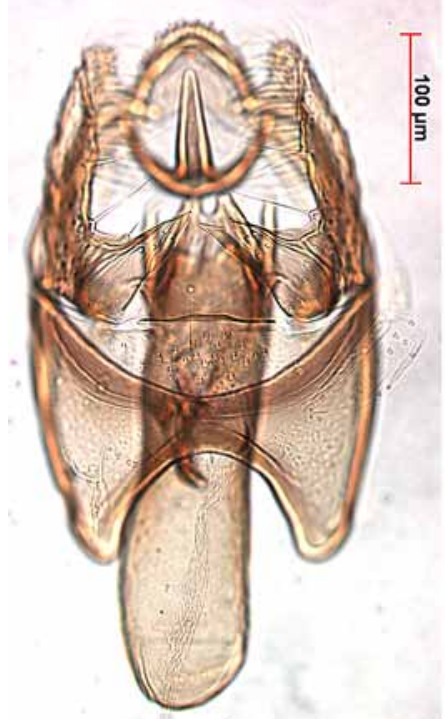

20

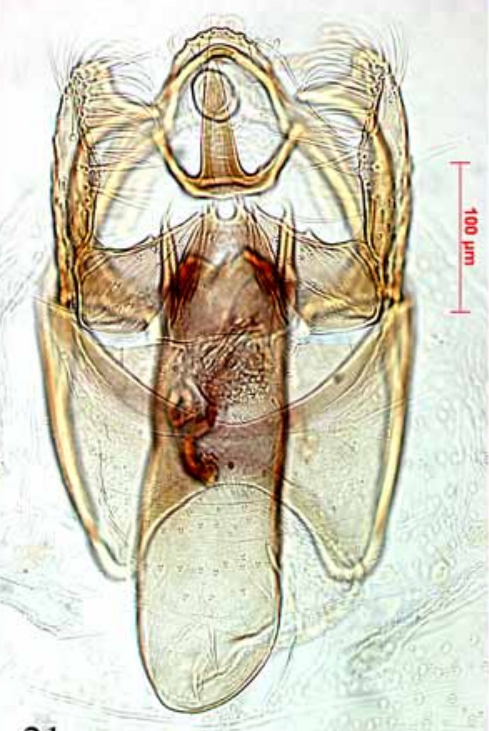

21

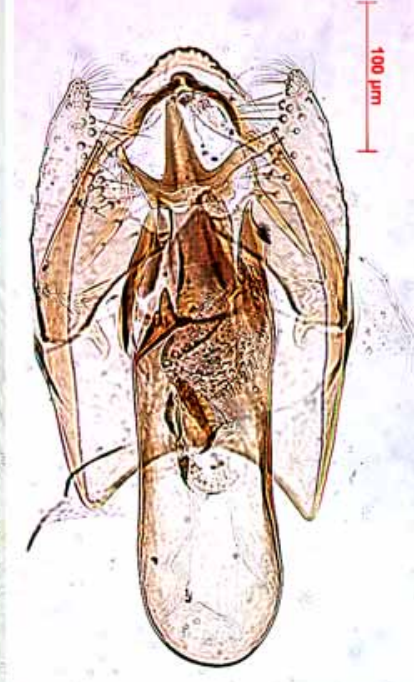

22

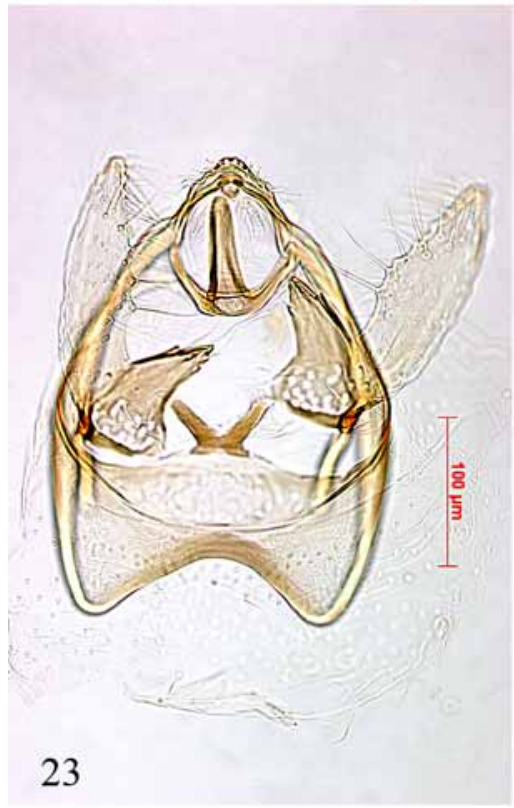

24

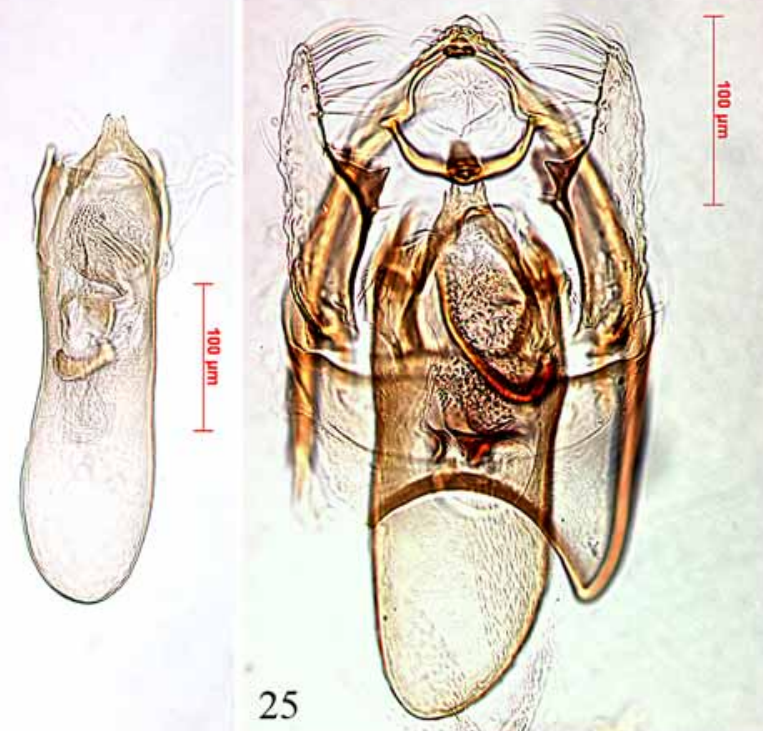

FIGURES 20-25. Acalyptris platani group: male genitalia in ventral view. 20-21, A. platani, slides 0828 and 1257, Italy, Trento; 22, A. loranthella, slide 1261, Greece, Evvoia; 23-24, A. minimella, slide 1433, France, Corsica, Porticcio, 25, A. pistaciae, slide 1269, Holotype. 

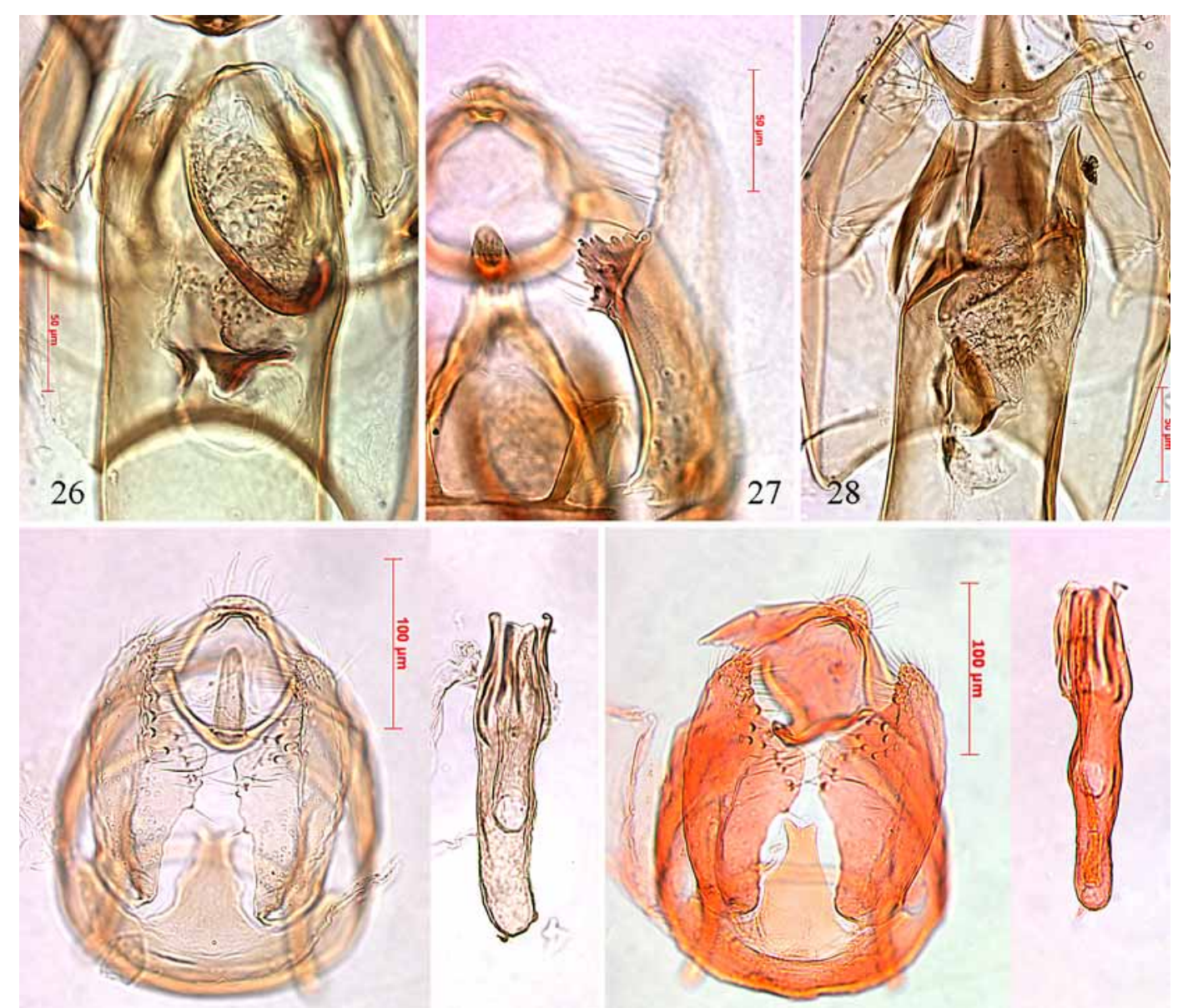

29

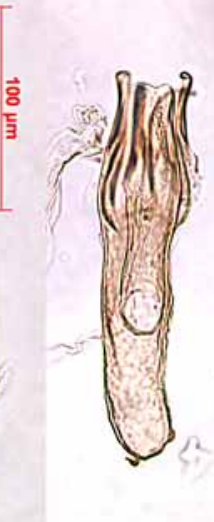

30
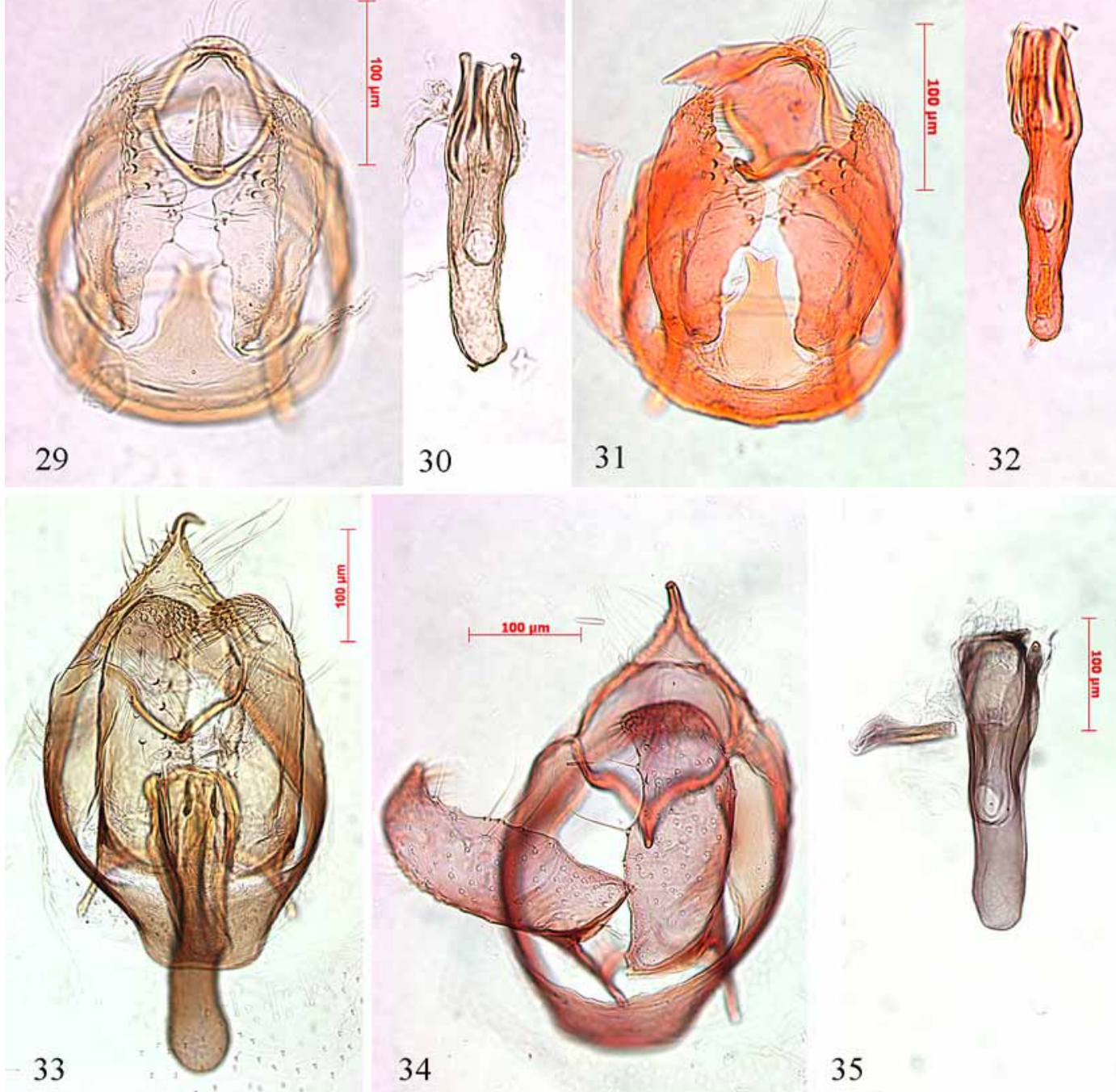

35

FIGURES 26-35. 26-28. Acalyptris platani group: male genitalia in ventral view, details. 26, 27, A. pistaciae, slide 1269, Holotype; 28, A. loranthella, slide 1261, Greece, Evvoia. 29-35. Acalyptris staticis group: male genitalia in ventral view. 29-32, A. staticis, slides 1430, Tenerife, Puerto de la Cruz, BM24095, Holotype; 33-35, A. limoniastri, slides 3127, Tunisia, Nefta, BM22597, Holotype and BM22616 (35), paratype, Algeria, Hammam-es-Salahin. 

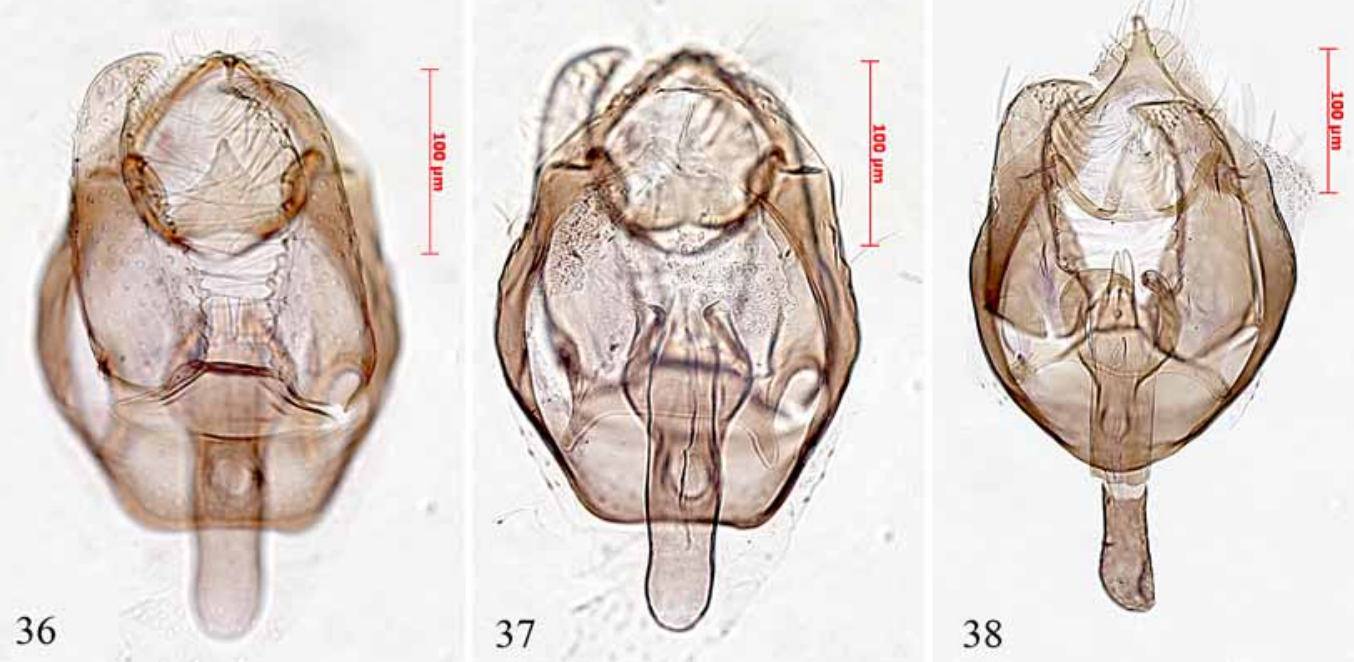

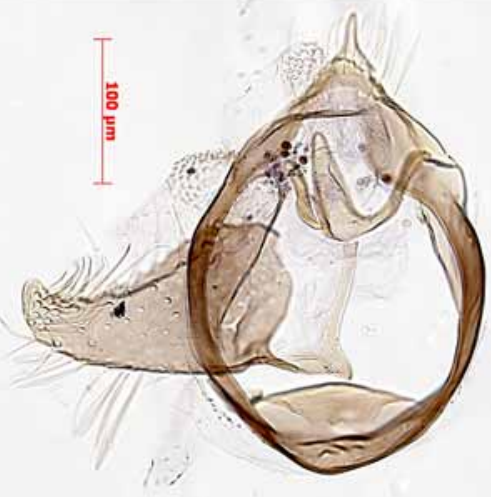

39

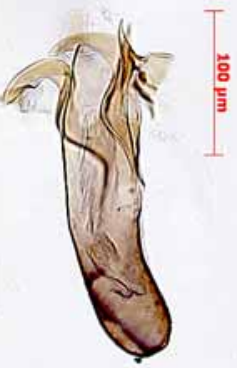

40
41

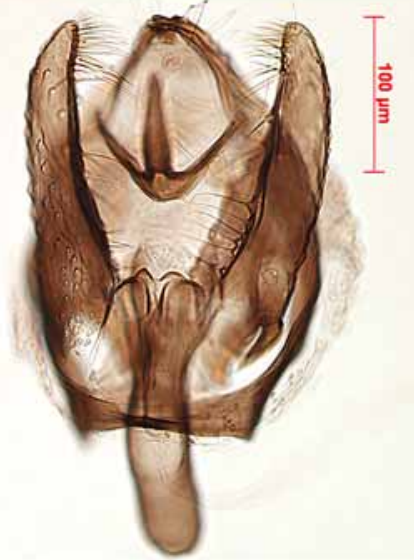

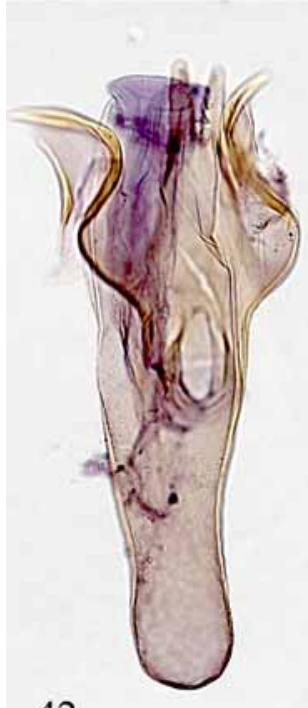

42

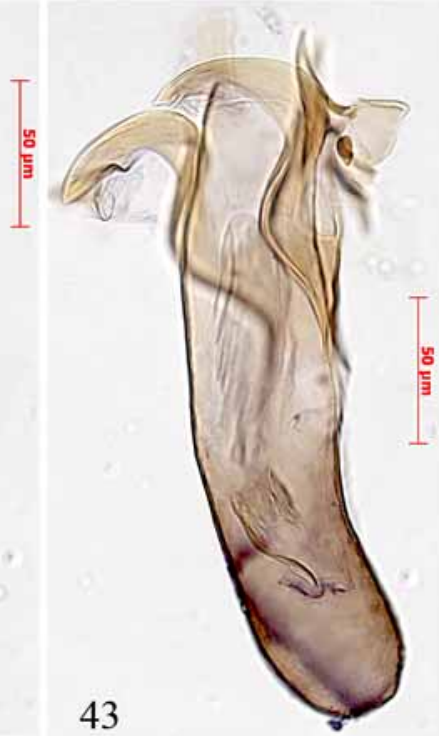

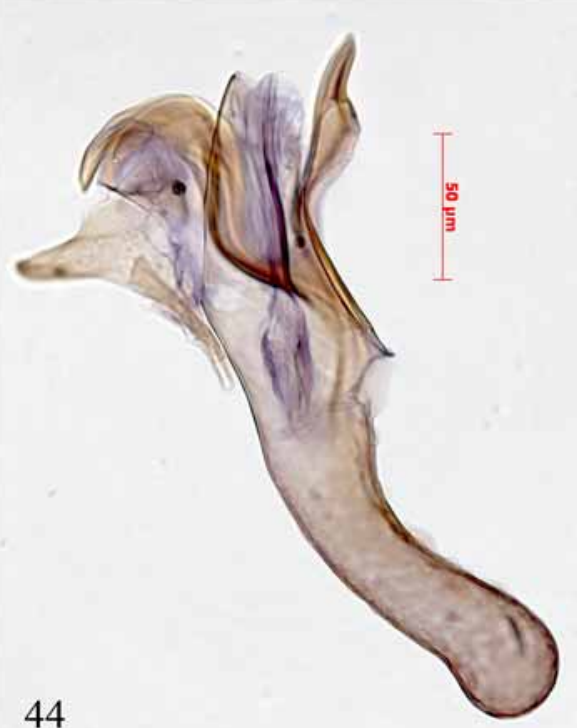

44

FIGURES 36-44. Acalyptris staticis group: male genitalia in ventral or lateral view (aedeagus, 40, 43, 44). 36, 37, 42, A. maritima, slides 3709, Italy, Portogruaro and 3515, Greece, Salaora, both paratypes; 38, 44, A. lesbia, slides 3625, Holotype and 3552, paratype 2003; 39, 40, 43, A. limonii, slide 3488, Greece, Salaora, paratype; 41, A. pyrenaica, slide 2827, Germany, Eifel, Nettersheim. 


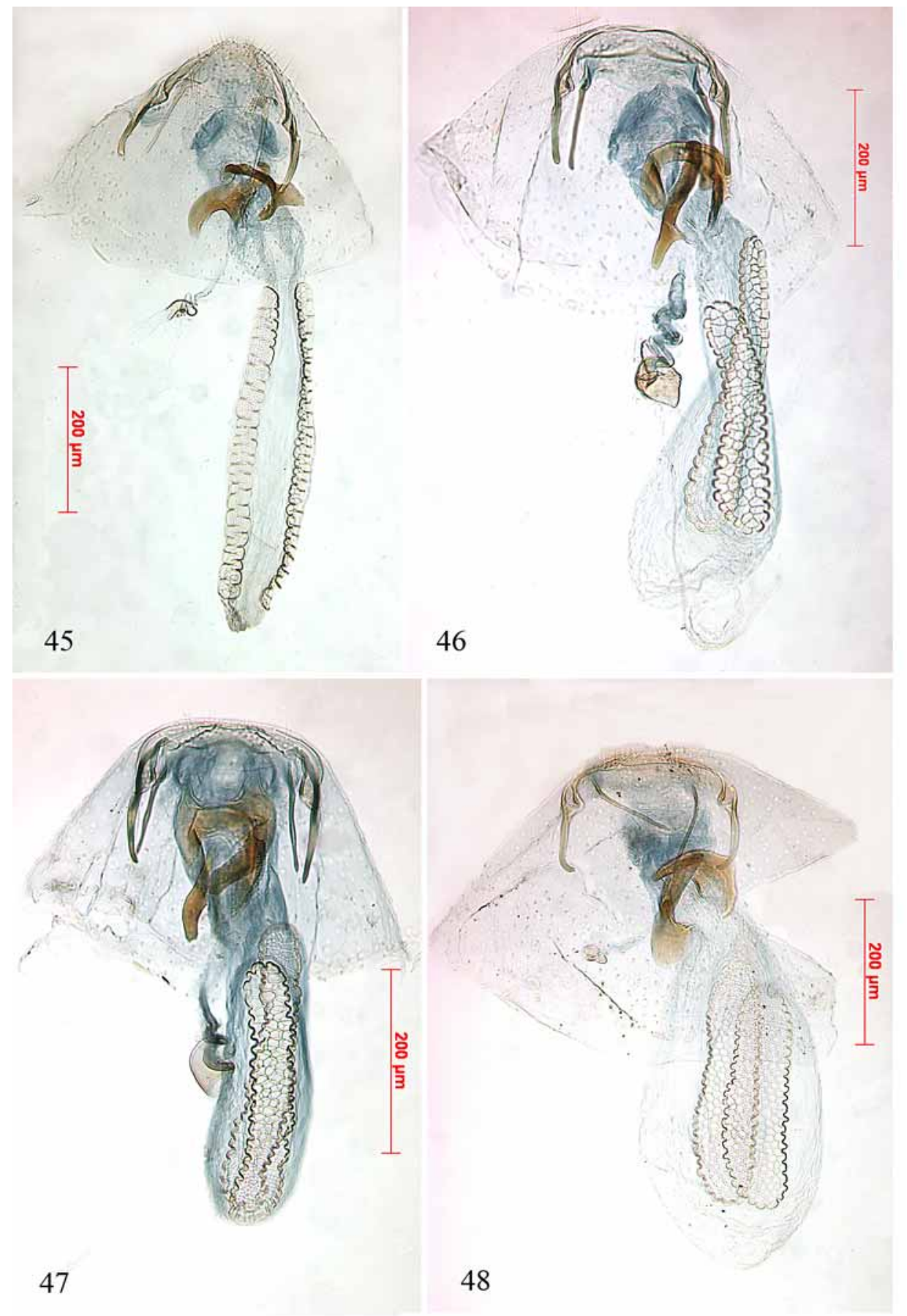

FIGURES 45-48. Acalyptris platani group: female genitalia in dorsal view. 45, A. platani, slide 1255, Italy, Trento; 46, A. minimella, slide 1265, Algeria, Morris; 47, A. pistaciae, slide 1266, Greece, Dhelfoi, paratype; 48, A. loranthella, slide 1260, Greece, Evvoia. 


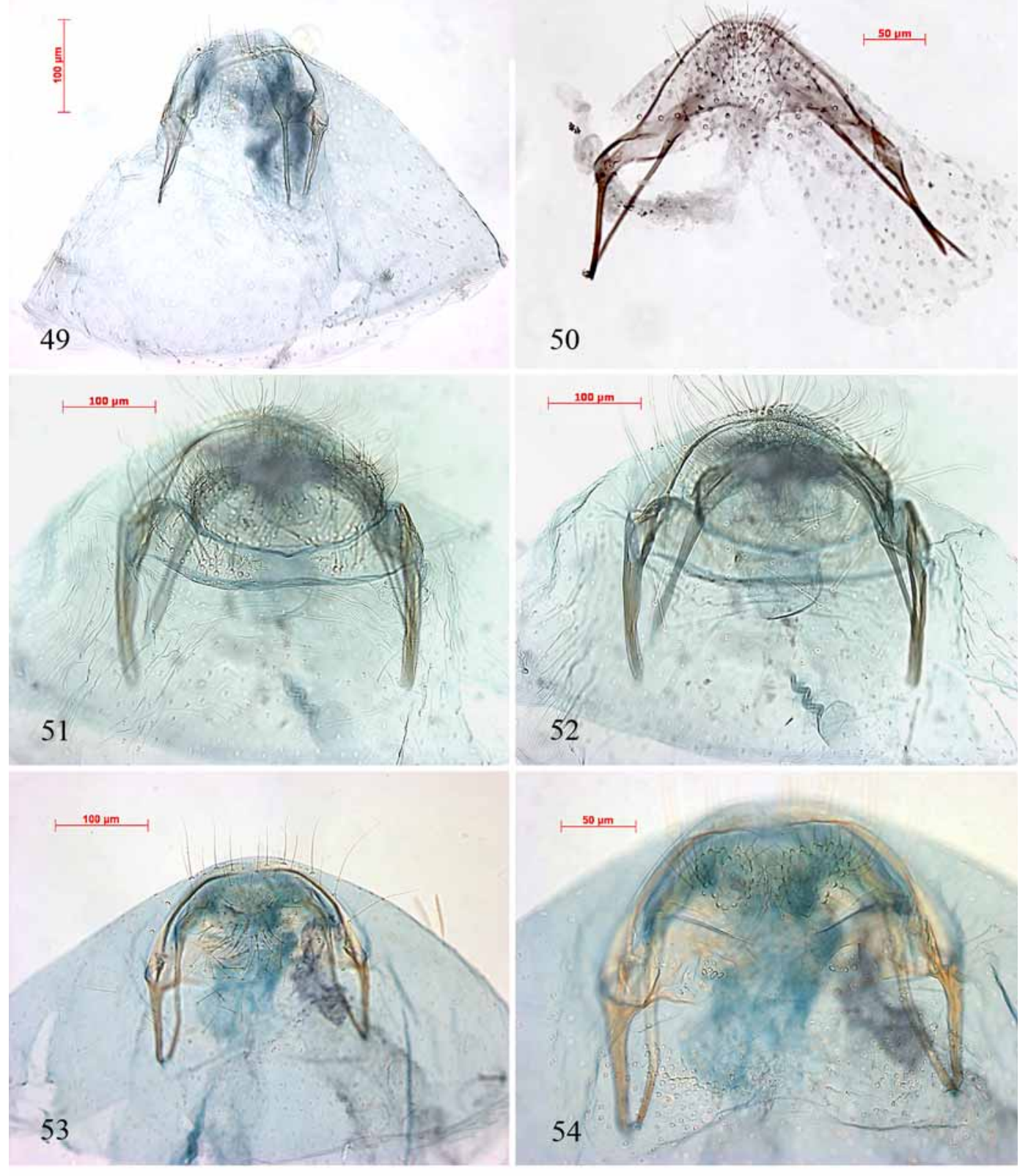

FIGURES 49-54. Acalyptris staticis group: terminal abdominal segments and female genitalia (as far as visible), in dorsal view and dorsally focussed $(49,51,54)$, more ventrally focussed, showing sternite $(50,52,53)$. 49, A. staticis, slide 1431, Canary islands, Tenerife, Puerto de la Cruz; 50, A. pyrenaica, slide 3569 (only partly preserved), Spain, Coll del Canto, paratype; 51, 52, A. limoniastri, slide BM22615, Algeria, Biskra, Paratype; 53, 54, A. limonii, slide 3529, Greece, Salaora, paratype. 


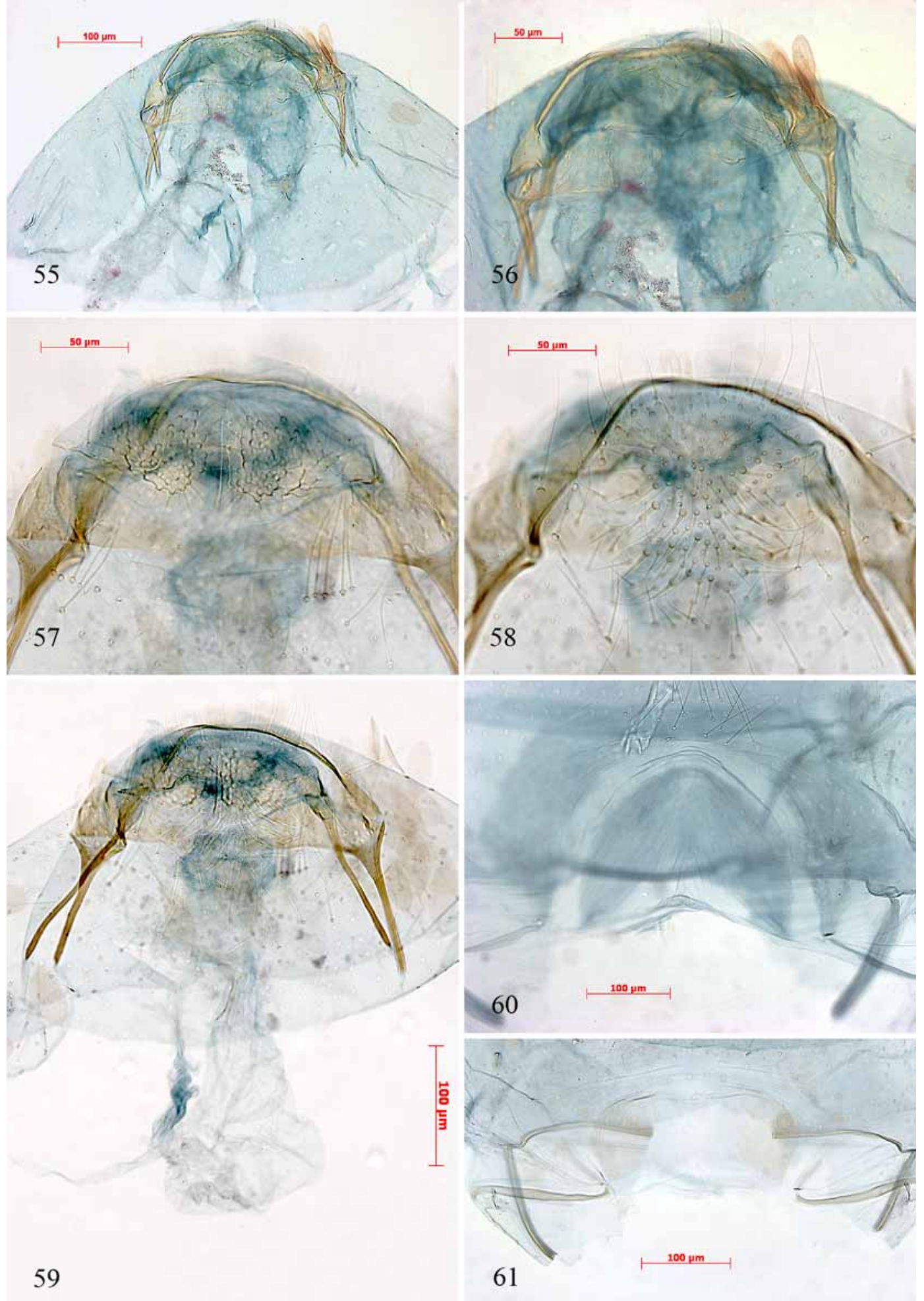

FIGURES 55-61. 55-59. Acalyptris staticis group: terminal abdominal segments and female genitalia (as far as visible), in dorsal view and dorsally focussed, in 58 more ventrally focussed, showing sternite. 55, 56, A. maritima, slide 3530 , Italy, Portogruaro; 57-59, A. lesbia, slide 3642, paratype, Greece, Lesvos. 60-61. Acalyptris species, female, second abdominal segment from ventral, showing shape of s2a. 60, A. limoniastri, slide BM22615, Algeria, Biskra, Paratype; 61, A. minimella, slide 3415, Portugal (Algarve), Serra de Monte Figo. 


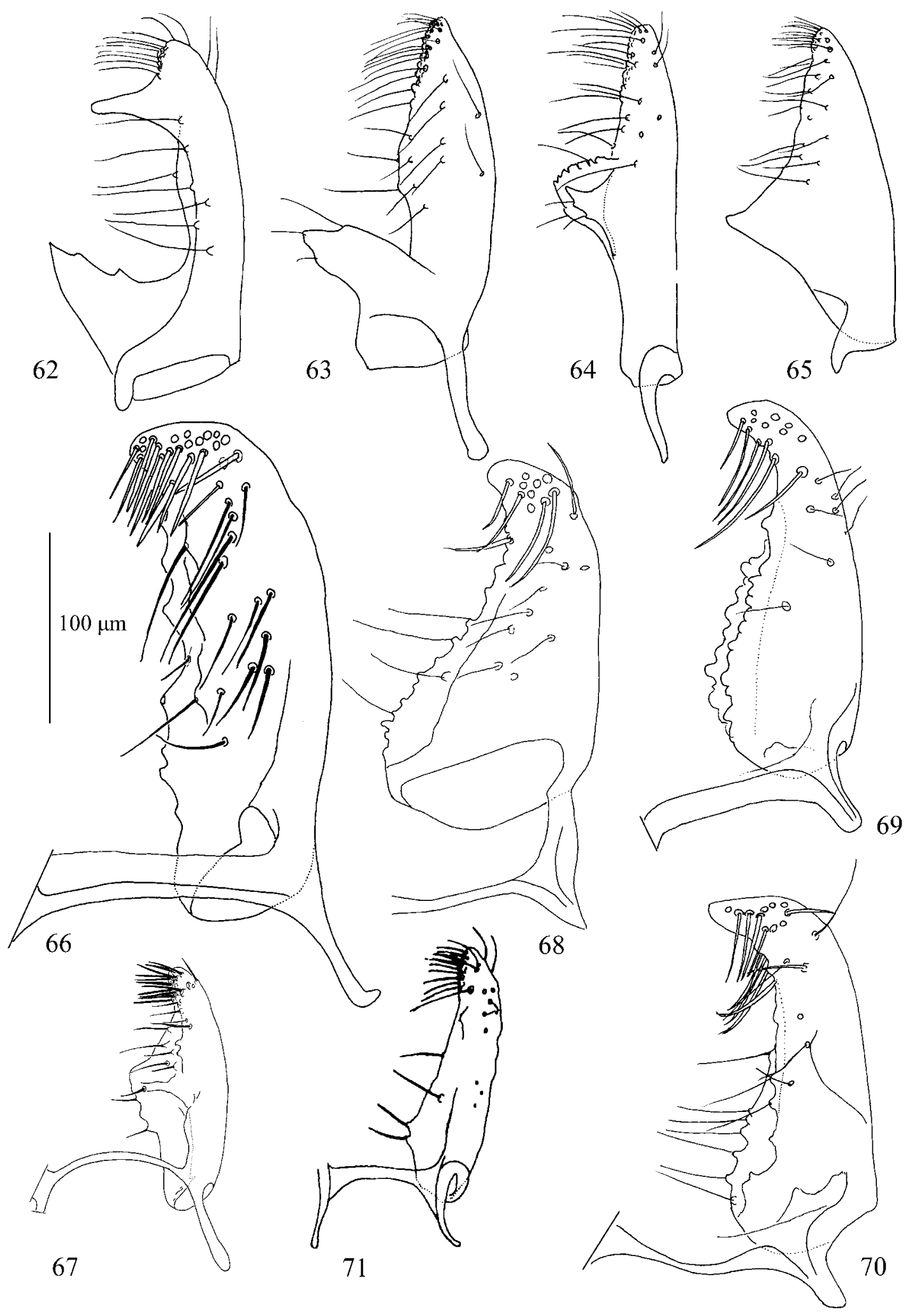

FIGURES 62-70. Acalyptris species: male genitalia, valva dorsal side, drawn from right valva, through ventral side (some from left valva, mirrored); not all setae on valval tip and margins are drawn. 62, A. platani, slide 1257, Italy, Trento; 63, A. minimella, slide 1433, Corsica, Porticcio; 64, A. pistaciae, 1268, Cyprus, Limassol, paratype; 65, A. loranthella, slide 1261, Greece, Evvoia; 66 A. limoniastri, slide BM22614, Algeria, Biskra, Paratype; 67, A. staticis, slide 1430, Canary islands, Tenerife, Puerto de la Cruz; 68, A. limonii, slide 3488, Greece, Salaora, paratype; 69, A. maritima, slide 3709, Italy, Portogruaro, paratype; 70, A. lesbia, slide 3625, male holotype, Greece, Lesvos; 71, A. pyrenaica, slide 2886, Germany, Eifel, Marmagen [not on same scale, from Nieukerken \& Biesenbaum (1997)]. 


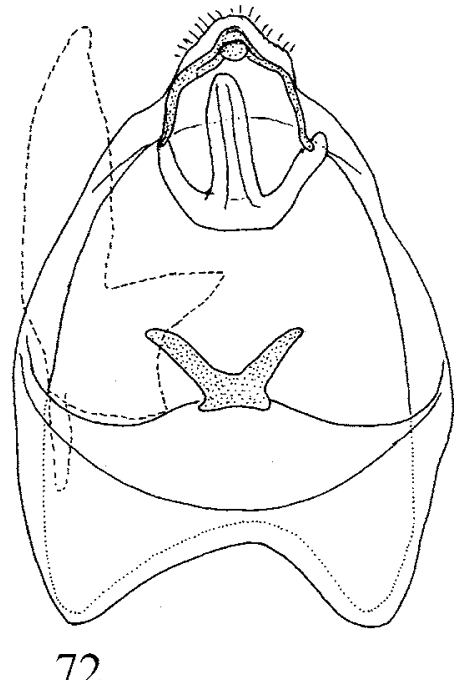

72
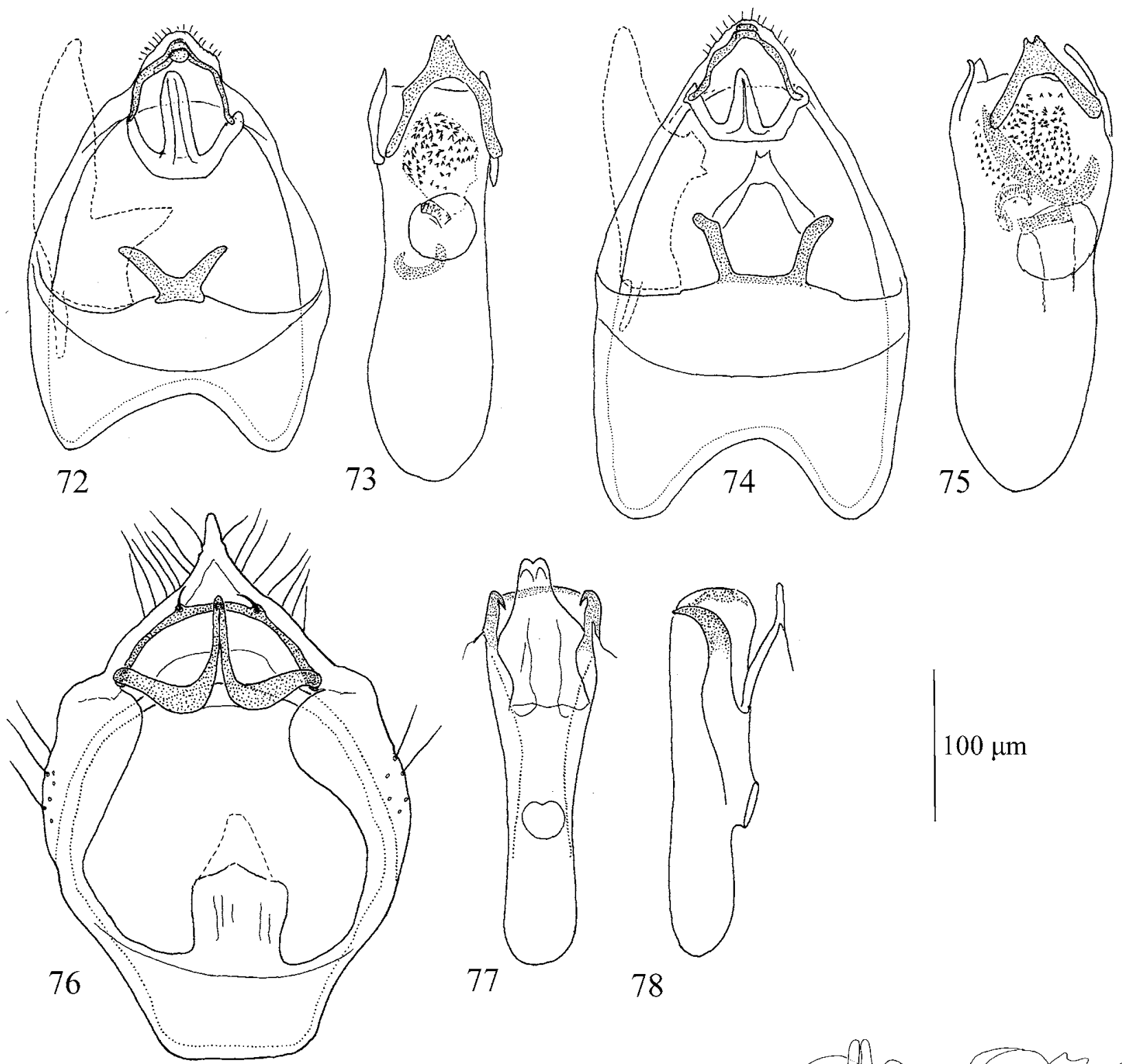

78
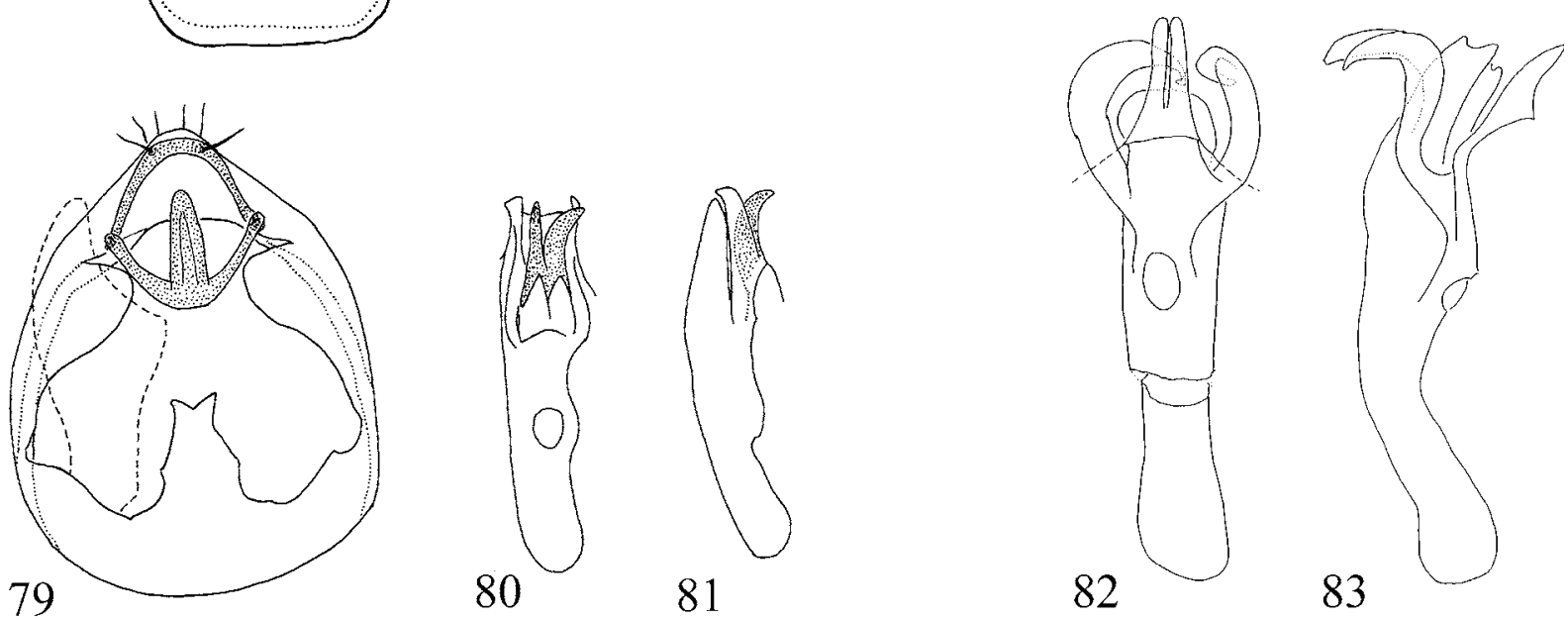

FIGURES 72-83. Acalyptris species, male genitalia, capsules $(72,74,76,79)$ and aedeagus in ventral $(73,75,7780$, 82) and lateral view (78, 81, 83). 72, 73, A. minimella, slide 1433, Corsica, Porticcio; 74, 75, A. pistaciae, slide 1268, Cyprus, Limassol, paratype; 76-78, A. limoniastri, slide BM22616, Algeria, Hammam-es-Salahin and BM 22613 (77), Biskra, paratypes; 79-81, A. staticis, slide 1430, Canary islands, Tenerife, Puerto de la Cruz; 82, 83, A .lesbia, Greece, Lesvos, slide 3625 , holotype and slide3552, paratype. 


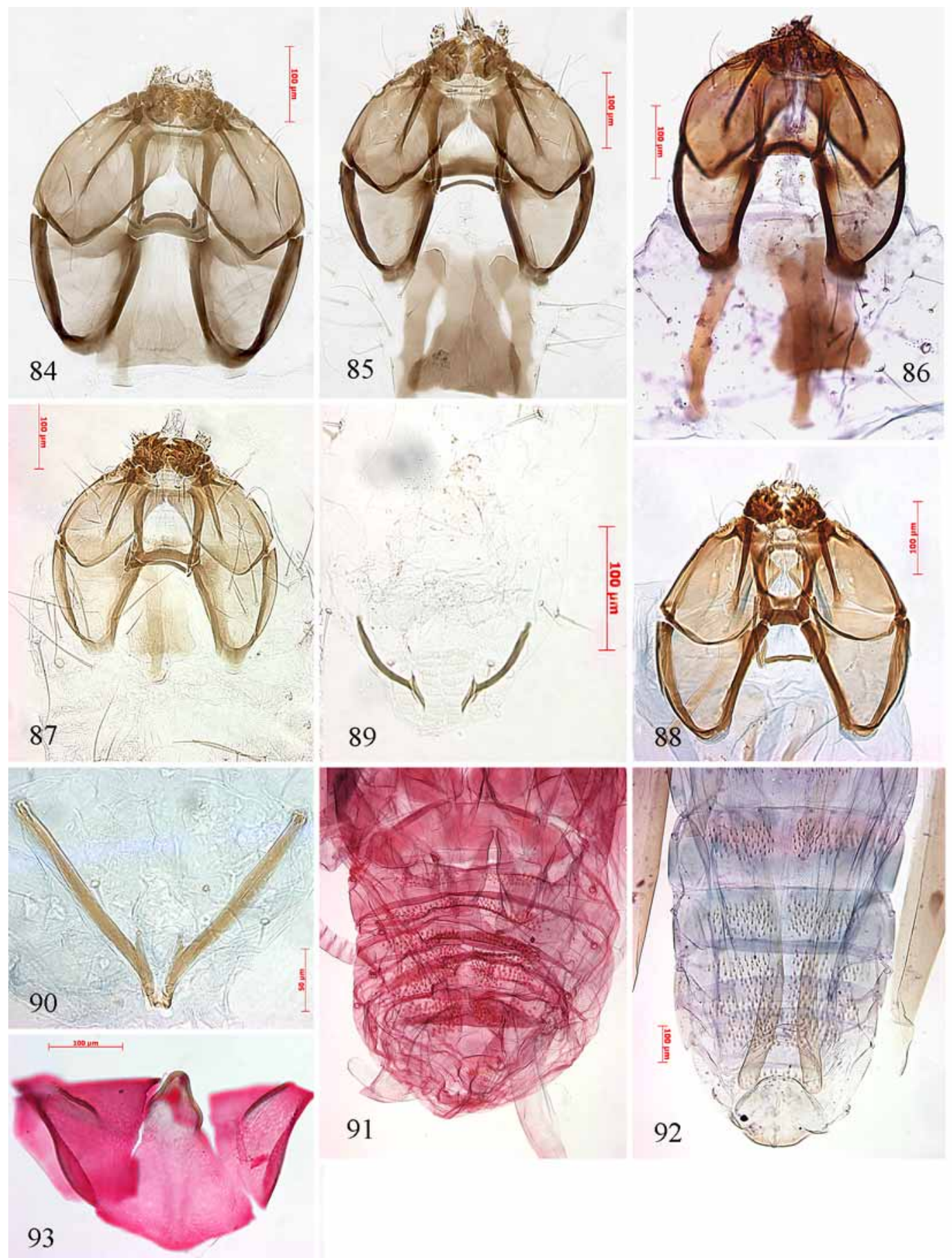

FIGURES 84-93. Acalyptris species, details of immatures. 84-88, Headcapsule of final instar larva and part of prothorax with sclerites. 89, 90, Last abdominal segment(s) with anal rods. 91-93, Pupal exuviae, details of abdomen with abdominal spines $(91,92)$ and detached frons with conical projection (93). 84, 89, A. platani, Greece, Evvoia, Dhírfis Oros; 85, A. minimella, Tunisia, Tabarka; 86, A.pistaciae, slide RMNH 11892, Turkey, Antalya, Selimiye; 87, A. loranthella, Greece, Evvoia, Dhírfis Oros; 88, 90, A. lesbia, RMNH 12393, Greece, Lesvos, paratype; 91, A. loranthella, slide 3647, Greece, Evvoia, Dhírfis Oros; 92, A. lesbia, slide 3643, Greece, Lesvos; 93, A. platani, slide 3646, Italy, Trieste. 

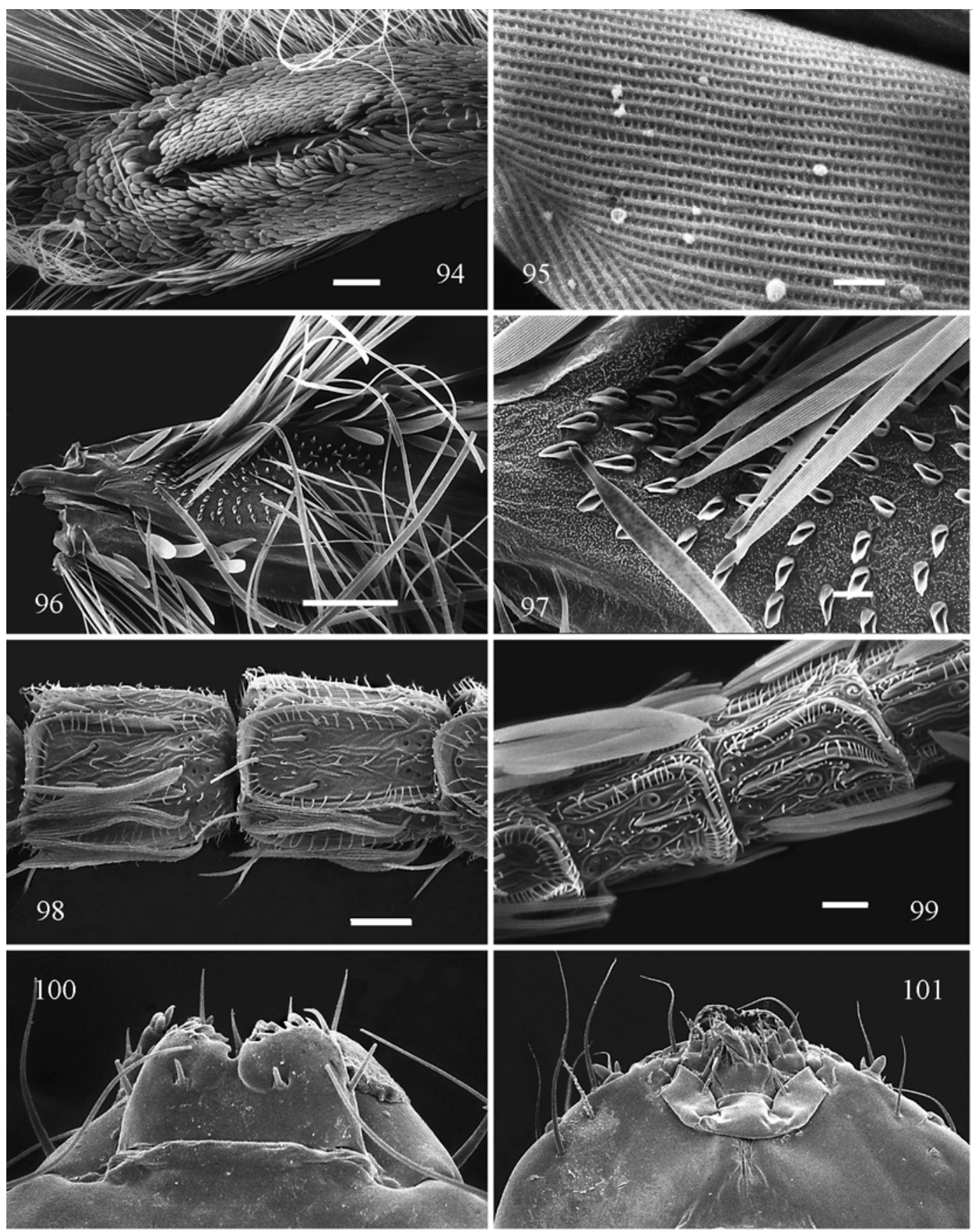

FIGURES 94-101. Acalyptris species, SEM micrographs, adult and larval structure. 94, 95, A. platani, male hindwing upperside with special scales and detail, Italy, Trento; 96, 97, A. pistaciae, male hindwing upperside with dispersed hairpencil; Greece, Dhelfoi; 98, 99, male flagellar segments with sensillum vesiculocladum: 98, A. platani, Italy, Trento; 99, A. limoniastri, Hammam-es-Salahin, paratype; 100, 101, A. minimella, larval headcapsule dorsal, detail of labrum and ventral, pictures taken at magnifications 950 and 760×, slightly reduced. Scales: $100 \mu \mathrm{m}(94,96), 10 \mu \mathrm{m}(97-99)$ and $1 \mu \mathrm{m}(95)$. 


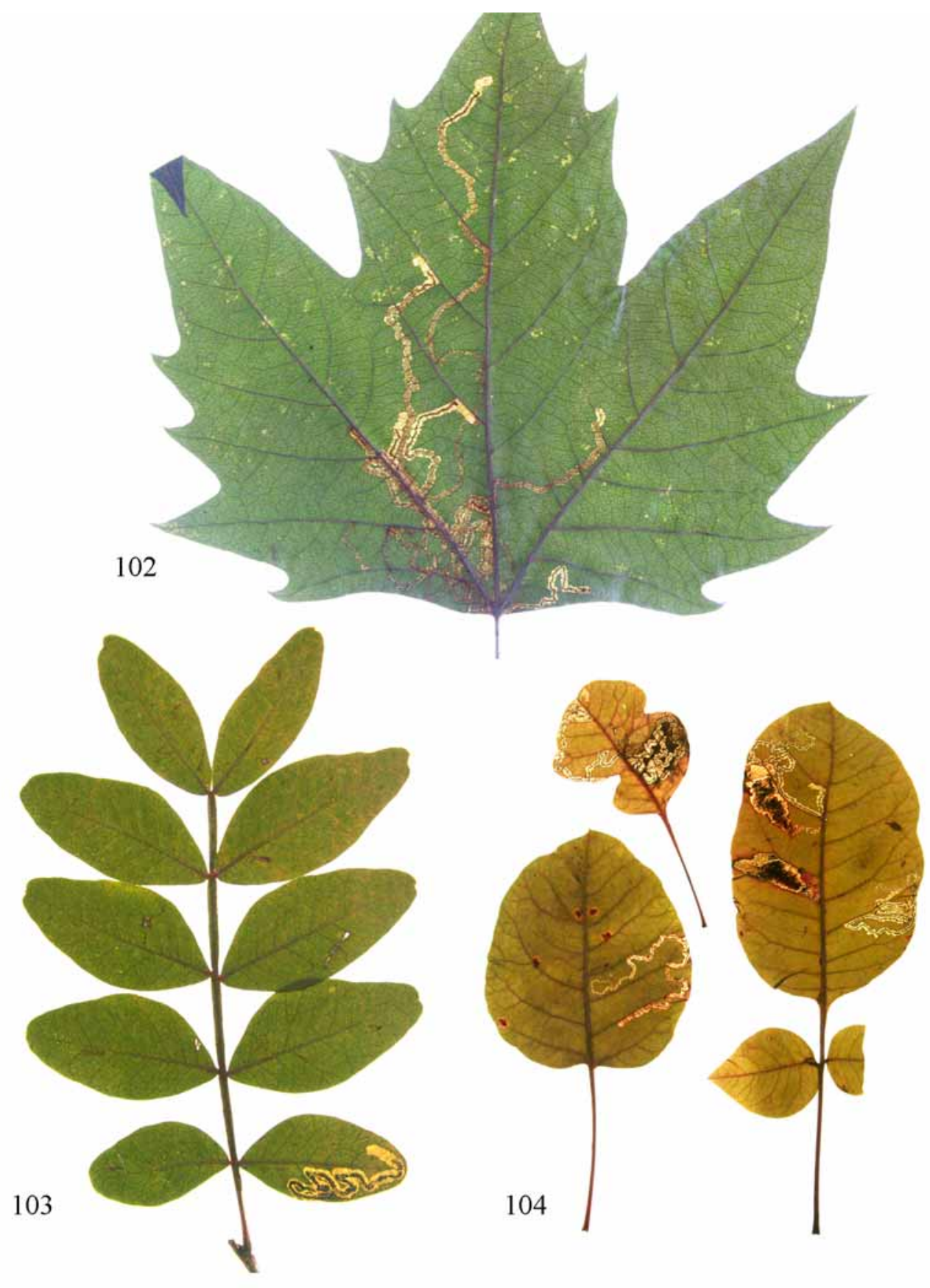

FIGURES 102-104. Acalyptris platani group, leafmines. 102, A. platani, France (Pyrénées-Orientales), Latour-deFrance; 103, A. minimella on Pistacia lentiscus, Spain, Sierra de Grazalema, El Bosque; 104, A. pistaciae on Pistacia terebinthus, Greece, Dhelfoi, type locality, together with mines of Ectoedemia terebinthivora. 


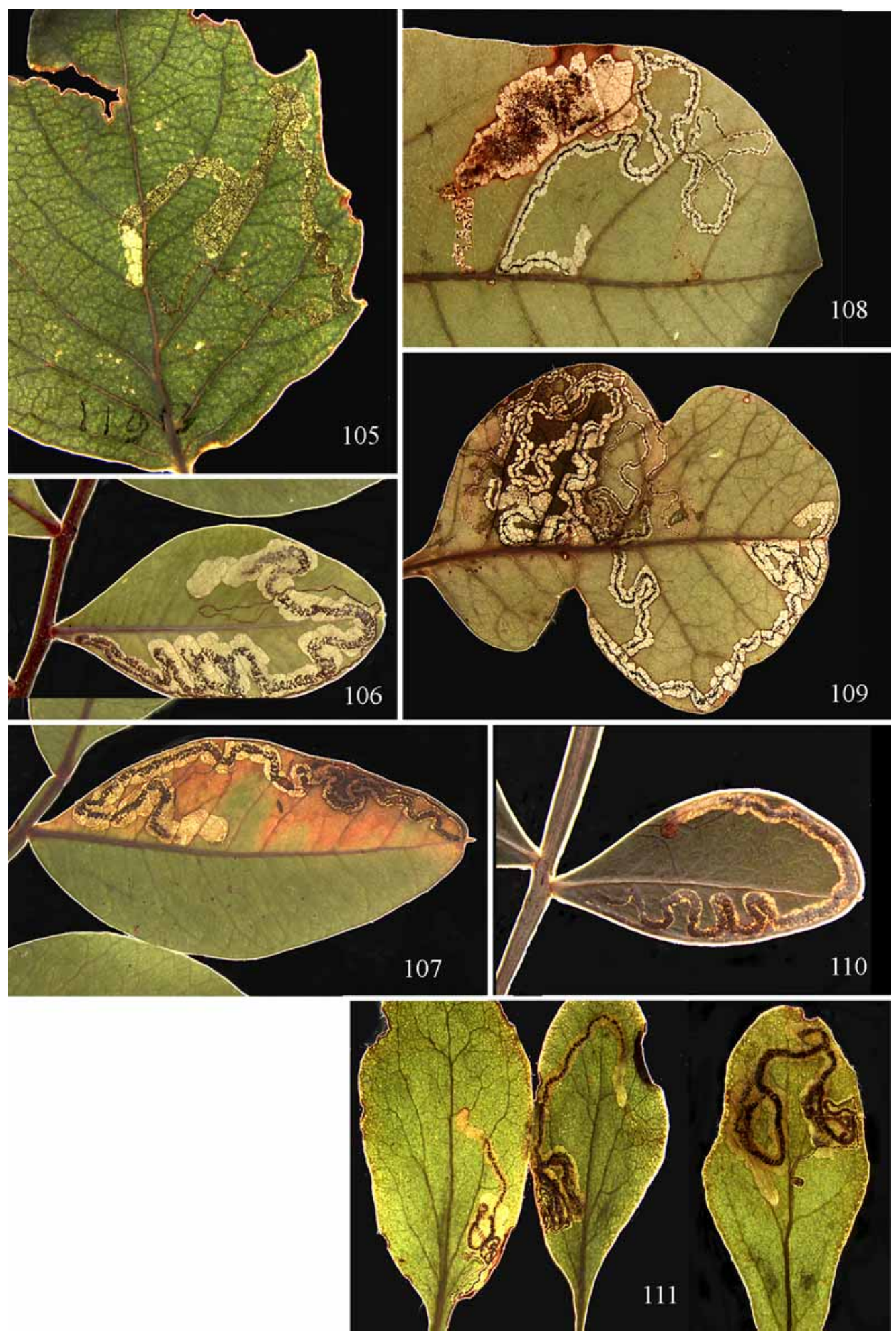

FIGURES 105-111. Acalyptris platani group, leafmines. 105, A. platani, France (Pyrénées-Orientales), Latour-deFrance, larva sequenced; 106, 107, A. minimella on Pistacia lentiscus, Spain, Sierra de Grazalema, El Bosque; 108, 109, A. pistaciae on Pistacia terebinthus, Greece, Dhelfoi, type locality, together with Ectoedemia terebinthivora; 110, A. pistaciae on Pistacia lentiscus, Cyprus, R. Johansson; 111, A. loranthella on Loranthus europaeus, Czech Republic, Moravia, Milovice. All mines $2.5 \times$ natural size. 

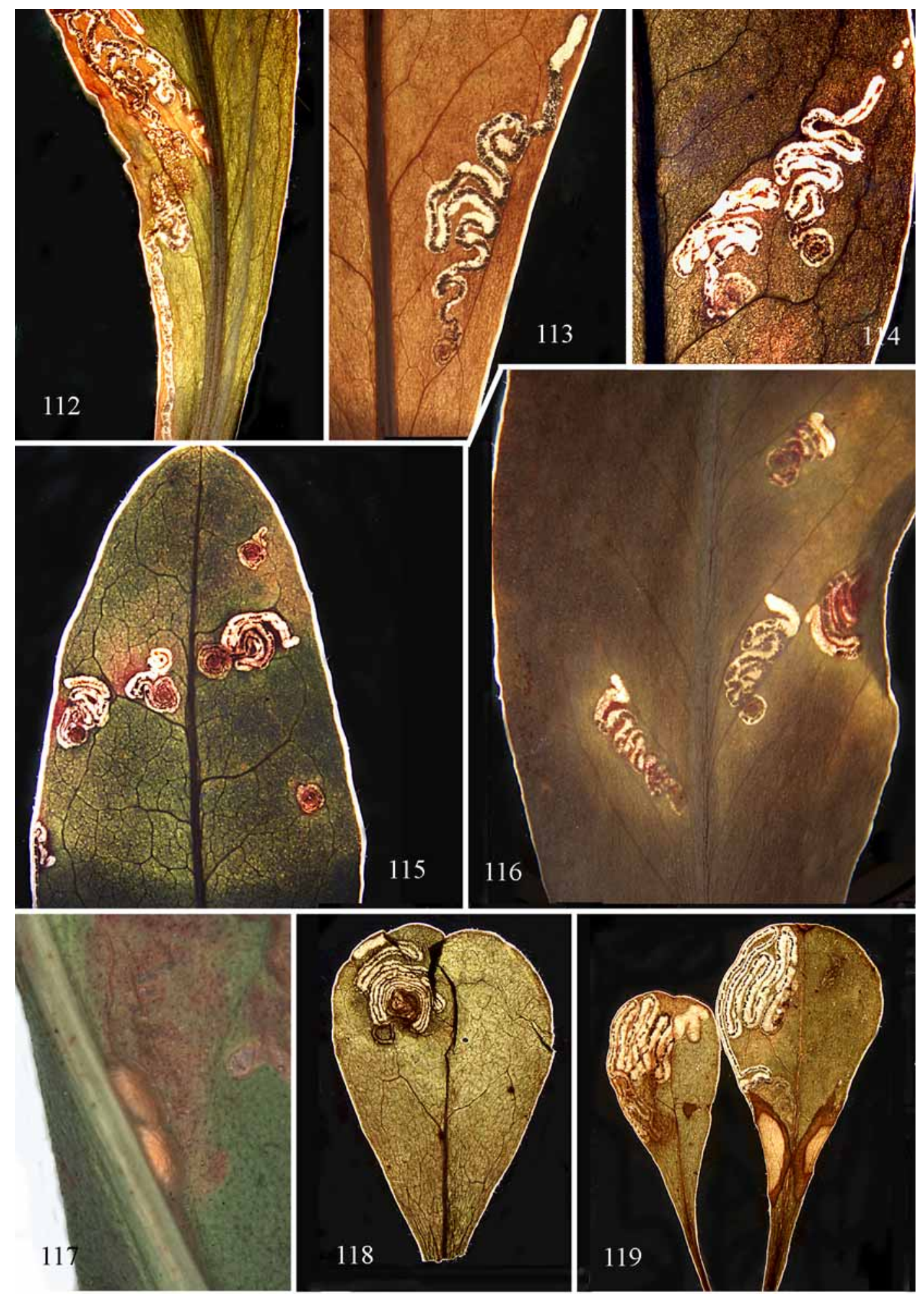

FIGURES 112-119. Acalyptris staticis group, leafmines. 112, A. maritima, Portogruaro, Brussa; 113, 114, A. limonii or maritima on Limonium sp., Arta, Salaora, 15.vi.1996; 115-117, A. lesbia on Limonium gmelini, 117 showing two cocoons on midrib, leaf underside; 118, 119, A. staticis on L. pectinatum, Tenerife, Puero de la Cruz. All mines $2 \times$ natural size, except Fig. 117, which is more enlarged. 

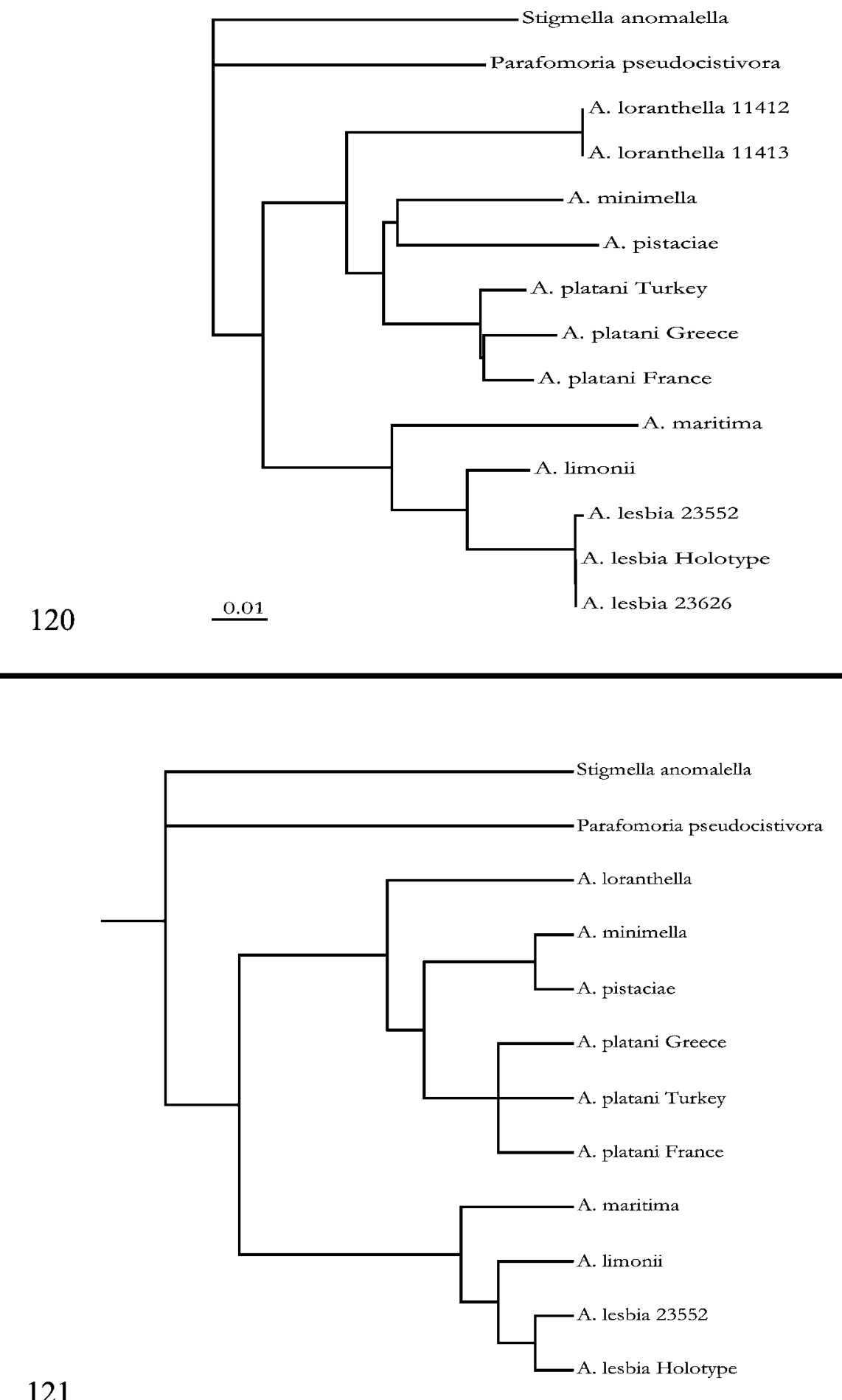

FIGURE 120-121. 120, Neighbour joining tree of all CO1 barcode sequences of Acalyptris and two outgroups, with relative distances (K2P distances). 121, Cladogram of Acalyptris and two outgroups based on the mitochondrial CO1 sequences: Strict consensus tree of three shortest trees found during exhaustive search $(\mathrm{CI}=0.683, \mathrm{RI}=0.685$, $\mathrm{RC}=0.468)$. 

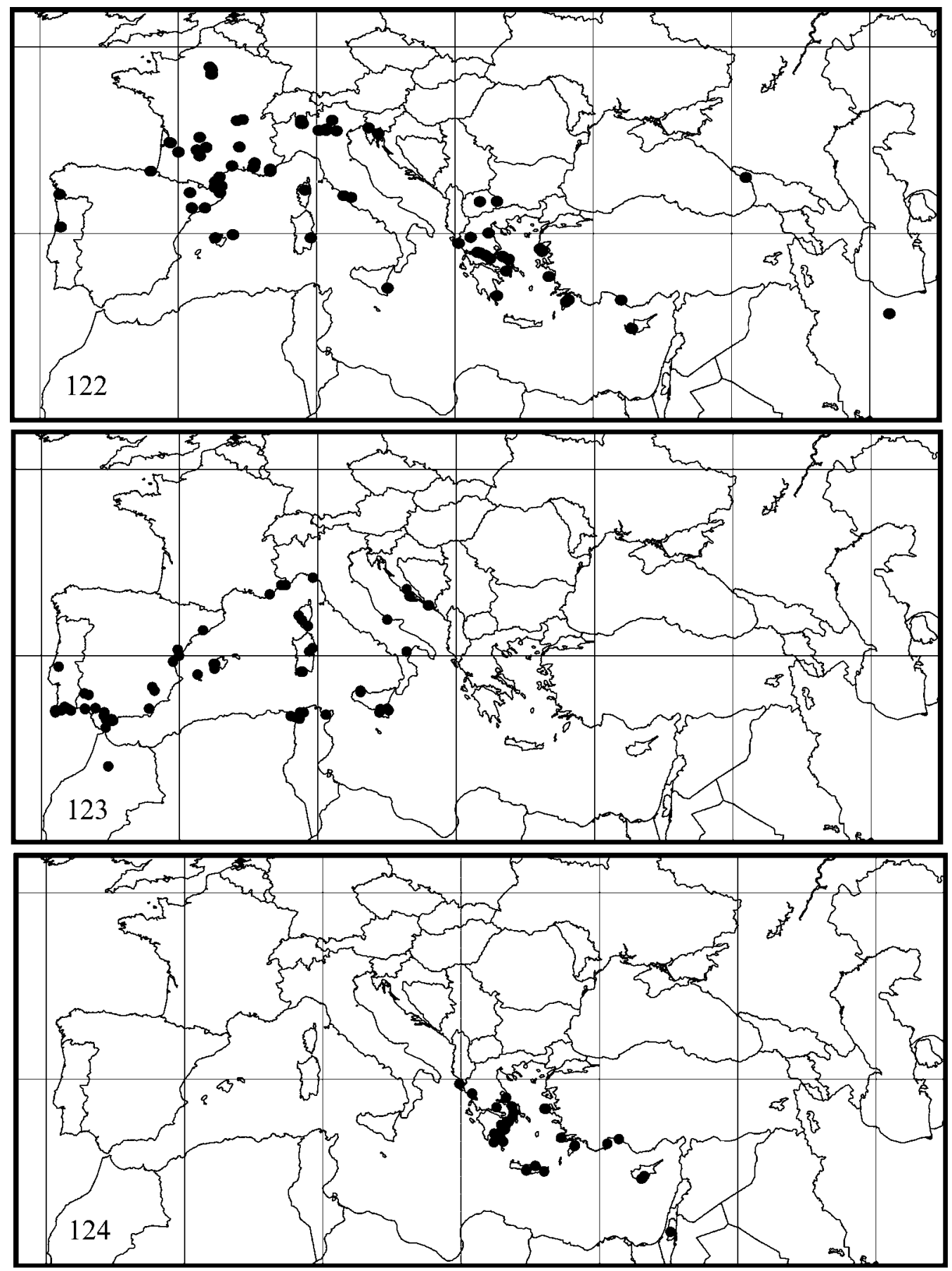

FIGURES 122-124. Distribution maps of Acalyptris. 122, A. platani; 123, A. minimella; 124, A. pistaciae. 

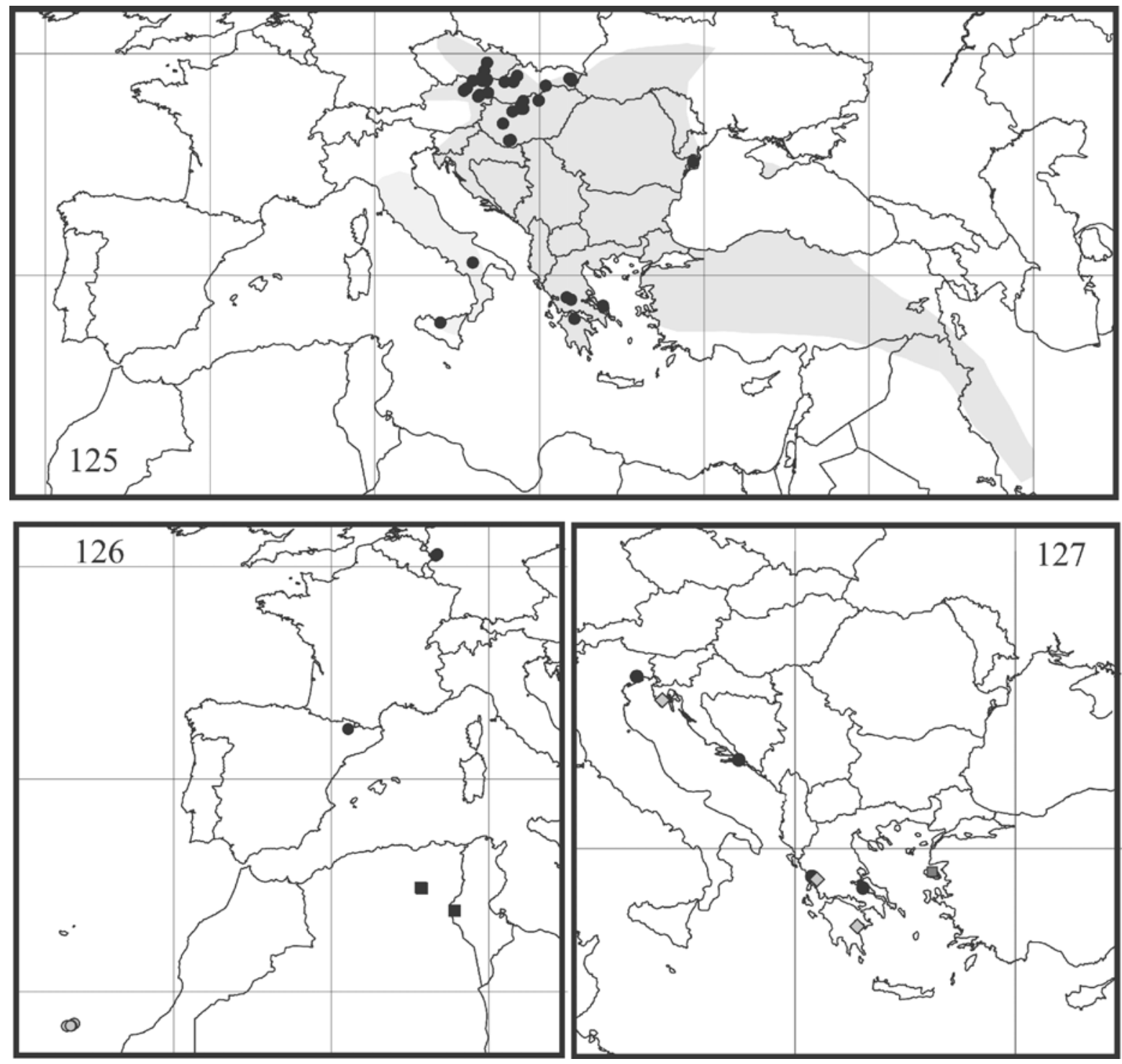

FIGURES 125-127. Distribution maps of Acalyptris. 125, A. loranthella, shaded area shows approximate distribution of host Loranthus europaeus; 126, A. staticis group in Western Mediterranean: A. pyrenaica (black dots), A. staticis (grey dots) and A. limoniastri (black squares); 127, A. staticis group in Eastern Mediterranean: A. maritima (black dots), A. limonii (grey diamonds) and A. lesbia (grey square). 